\title{
Bypassing the Blood-Brain Barrier: A Physical and Pharmacological Approach for the Treatment of Metastatic Brain Tumors
}

\author{
Samuel A. Sprowls \\ West Virginia University Health Sciences Center
}

Follow this and additional works at: https://researchrepository.wvu.edu/etd

Part of the Animal Experimentation and Research Commons, Cancer Biology Commons, Medicinal Chemistry and Pharmaceutics Commons, Neoplasms Commons, Neurosciences Commons, Oncology Commons, Other Neuroscience and Neurobiology Commons, Other Pharmacy and Pharmaceutical Sciences Commons, and the Other Physical Sciences and Mathematics Commons

\section{Recommended Citation}

Sprowls, Samuel A., "Bypassing the Blood-Brain Barrier: A Physical and Pharmacological Approach for the Treatment of Metastatic Brain Tumors" (2021). Graduate Theses, Dissertations, and Problem Reports.

8261.

https://researchrepository.wvu.edu/etd/8261

This Dissertation is protected by copyright and/or related rights. It has been brought to you by the The Research Repository @ WVU with permission from the rights-holder(s). You are free to use this Dissertation in any way that is permitted by the copyright and related rights legislation that applies to your use. For other uses you must obtain permission from the rights-holder(s) directly, unless additional rights are indicated by a Creative Commons license in the record and/ or on the work itself. This Dissertation has been accepted for inclusion in WVU Graduate Theses, Dissertations, and Problem Reports collection by an authorized administrator of The Research Repository @ WVU.

For more information, please contact researchrepository@mail.wvu.edu. 


\section{Bypassing the Blood-Brain Barrier: A Physical and Pharmacological Approach for the Treatment of Metastatic Brain Tumors}

Samuel A. Sprowls

Follow this and additional works at: https://researchrepository.wvu.edu/etd

Part of the Animal Experimentation and Research Commons, Cancer Biology Commons, Medicinal Chemistry and Pharmaceutics Commons, Neoplasms Commons, Neurosciences Commons, Oncology Commons, Other Neuroscience and Neurobiology Commons, Other Pharmacy and Pharmaceutical Sciences Commons, and the Other Physical Sciences and Mathematics Commons 


\title{
Bypassing the Blood-Brain Barrier: A Physical and Pharmacological Approach for the Treatment of Metastatic Brain Tumors
}

Samuel A. Sprowls, B.S.

\author{
Dissertation submitted \\ to the School of Pharmacy \\ at West Virginia University
}

in partial fulfillment of the requirements for the degree of

Doctor of Philosophy in Pharmaceutical and Pharmacological Sciences

\author{
Paul R. Lockman, Ph.D., Chair \\ R. Alfredo C. Siochi, Ph.D., \\ Werner Geldenhuys, M.D., Ph.D., \\ Lori A. Hazlehurst, Ph.D., \\ Marina Galvez, Pharm.D.
}

Department of Pharmaceutical and Pharmacological Sciences Robert C. Byrd Health Sciences Center

\author{
Morgantown, West Virginia \\ 2021
}

Keywords: Brain metastases, breast cancer, radiation therapy, blood-tumor barrier, chemotherapy, permeability.

Copyright 2021 Samuel A. Sprowls 


\section{ABSTRACT \\ Bypassing the Blood-Brain Barrier: A Physical and Pharmacological Approach for the Treatment of Metastatic Brain Tumors}

\section{Samuel A. Sprowls}

This dissertation (a) provided an in depth literature review of methods to disrupt the BBB/BTB and improve therapeutic distribution to brain tumors, (b) evaluated the use of azacitidine as a single agent therapy for the treatment of brain metastasis of breast cancer and a potential molecular mechanism by which brain tropic cells are sensitized to hypomethylating agents, (c) determined the impact cannabidiol has on P-glycoprotein mediated efflux at the blood-brain barrier and its potential for use as a single agent treatment for metastatic brain tumors, (d) developed a preclinical radiation therapy protocol for use in small animals and in vitro systems, (e) evaluated the impact radiation therapy has on blood-brain barrier integrity in normal and pathological brain, and (f) provided a discussion on the mathematical models used to evaluate blood-brain barrier pharmacokinetics in both normal and pathophysiological conditions. 


\section{ACKNOWLEDGEMENTS}

The work presented in this dissertation was possible not because of a single effort by myself, but as a collaborative effort supported by the many people I have been fortunate enough to know throughout my life.

First, and foremost I want to thank my wonderful wife. Completing this degree has been a dream of mine for as long as I can remember. In fact, shortly before I met you I vowed that I would never date another young woman until after I had my medical doctorate completed. Looking back now that just seems silly. I can remember passing by $\mathrm{NIOSH}$ on some of our first dates and talking about how I wanted to be like them, I wanted to be a scientist. From the time we met you have not only supported my scientific exploits, but encouraged and accepted this part of me. This journey would be simply impossible without you. You are the single most important thing in my life and I hope that I have made you proud to call me your husband. Over the last few years, you have fulfilled every aspect of the vows we made to each other. You have been there in the good and the bad times, through the frustrating failed experiments and to share in the insurmountable joy of my successes. You have been there when I felt pressured to quit and when I felt like I was unworthy of the faith you and those around me put forth. From the first step into graduate school and to my last day at WVU as a graduate student, you have done nothing but encouraged and believed in me. I cannot put into words how thankful I am to have had you by my side through this 
adventure. I am beyond excited to see where this crazy life takes us. I love you wife.

I would like to profoundly thank my mentor Dr. Paul Lockman. Your encouragement and support has meant the world to me. From literally the first encounter I had with you, I have felt as if I can do anything. You took a chance on me when it seemed as if no one else would. I cannot thank you enough for that. I am in the position I am today because of you. You have encouraged me to take leaps of faith without the fear of failure. Your inspiration and mentorship is not something I will forget. I have learned so much from you over the last few years. The balance that you have between work and life is something that I find fascinating and hope to model in my own life someday. You are the standard I measure myself against in science and in life. I would be happy if I end up being even half as successful as you have become. My time at WVU in graduate school has honestly been the best years of my life. I owe that all to you. Thank you, for everything.

To my dissertation committee, Dr. R. Alfredo C. Siochi, Dr. Werner Geldenhuys, Dr. Marina Galvez, and Dr. Lori Hazlehurst. In more ways that being a committee member, you all have been there guide me when necessary. Thank you all for your patience and your commitment to my graduate training. Your advice and recommendations for experiments has been more helpful than I am able to 
describe. I thank you sincerely, and hope that in your eyes I am on the way to becoming a scientist you are proud of.

To my senior lab mates. Having someone to learn from and to guide you in the right direction is a necessity for someone pursuing a $\mathrm{PhD}$. Neal Shah and Afroz Mohammad could not have fit this role better. Neal, you have gone from the person I could not have felt more inferior of to one of my closest friends. We have had our ups and downs, and differences of opinions, but when it mattered most, you were there to help. I think we can finally agree that while science may be one our commonalities, a love for Game of Thrones is what clearly brought us closer together. I am excited to see where you career takes you; Congratulations on getting accepted to WVU Dermatology for your residency, and surviving your first year! Afroz, I cannot remember a time when I finished speaking to you and did not turn away smiling. You definitely kept Neal and I on the right track and brought us back to a positive mindset if we wandered. Your intelligence and kindness definitely were an inspiration, and proved that a scientist does not have to be arrogant or unapproachable to be successful. I thank you both for all that I was able to learn from and experience with you. I owe you both a great deal.

To my fellow classmate, Pushkar Saralkar. Our friendship could not have started in a better way. You literally crashed my interview with our mentor, Dr. Lockman. I will never forget that interview weekend! You referred to me as the only American who loved soccer, and didn't actually prefer "football". Our friendship 
has grown much more since that weekend. It almost seems like we have been tied at the hip since we have started graduate school. Through foundations, all of the many courses, seminars, and experiments, and life in general we have done it all together. I wish you the best in life and I am very excited to see were life takes you. If you don't keep in touch, I will find you. Good luck in Minnesota!

To my fellow Lockman lab PhD. students, Tasneem Arsiwala, Kat Blethen, and Ross Fladeland. When in graduate school, I think it is hard to understand how close you will become to those around you. You three have become part of my second family over the last few years. Our lab could not be in better hands moving forward. You both have pushed me to become a better scientist and senior lab student. When Neal and Afroz left, I was thrust into a situation where I was not sure I could handle the responsibility of leading our lab in their absence. Tasneem you jumped right in from the start and helped me understand how much I have truly learned. Without you, I doubt I would be in the position I am today. You have helped me immensely on almost every facet of this work. I am so excited to see the results of our work with ExAblate and to see where your career takes you! Kat, I am glad you decided to join our lab! You are going to revolutionize the work we do. Thank you for pushing me to be more organized and to relearn immunology. Just to set the record straight, when I first thought of going to graduate school, it was for Immunology! Don't lose your competitive spirit and keep pushing. You are going to push the blood-brain barrier field to new heights. Ross, I have never met anyone near as positive as you. Having you 
around the last year has been great. You keep me on my toes and call me out on some of my worst ideas. Keep bringing the best you can each day and pushing us all to do better. I can't wait to see where your project takes you. Thank you all, it has a great honor to be your student mentor and friend. I am so excited to see where science guides you all!

To my favorite collaborators and friends, Dr. William Walker and Jacob Bumgarner. You two were essential for my graduate training, not only for science, but as my closest confidants. William, you were a mentor even if you don't' realize it. Watching you finish graduate school and progress through your post-doc phase has been insightful and I hope that I someday can be as efficient and knowledgeable as you. I can't wait to see where you land as professor in the near future! Jacob, I am sorry for being the reason people call you Jake. You didn't deserve that. You are an amazing friend and colleague. It has been great to see you go from a stellar musician to an even better scientist in training. Your enthusiasm and willingness to pursue science has been an inspiration. Thank you both for all you have helped with and allowed me to be part of! I hope that our paths cross as much as possible from here!

To the many undergraduate students that I have had the pleasure of working with. Thank you all for putting up with me and for being there to help when needed. You all are amazing and the many thank you notes that I should make would be a book on its own. Brooke Kielkowski, I don't know how to thank you 
enough for your work and friendship. You were there in a time where I was at my lowest point in graduate school, and when I thought for certain that I would never graduate. However, we made it work for a time when we were the only two in our lab! Thank you, and good luck during the rest of your time in pharmacy school and beyond! Morgan Glass, thank you for being around to help whenever needed. Your kind and positive attitude have been great to have around. I apologize for constantly changing things up on you. You are a real champ. Good luck with your future, I look forward to seeing all that you accomplish! I hope that I have provided you all with adequate scientific and life mentorship. I will surely miss you all. Good luck!

To the many cores facilities and OLAR employees, and other lab groups that have helped me along the way, thank you. Sarah McGlaughlin and Amanda Ammer. Thank you for literally everything. Every single figure I have ever made, at some point came from the advice and information you were happy to provide. You both have been so patient with me over the last few years. I am beyond grateful for all that you have done. I consider you both to be two of the people I have grown to know the most since my time here. I will surely miss sharing coffee and banter with you! Ethan Galand, you will forever have my gratitude. Of the many, many mice I have worked with, you have been there to keep me in line and to help whenever needed. You sir were a necessity for my graduate training and I am delighted to know you. Debra Piktel, or as I like to call you, lab mom. I am undeserved of your kindness. You have been in my corner from the time we 
first met. I thank you so very much for being a friend and someone to look up to, not only in science, but in life. You are an amazing person and I am lucky to have had you close the last few years. Basketball season will surely never be the same anywhere else!

To my extended family, Bethan, Jonathan, Cole, and Ellee Owen. I can't say thank you enough. From the time Kelsey and I started seeing each other you have been nothing but kind and supportive, and sometimes challenging, but I wouldn't have it any other way. I am blessed to be able to have had you by my side during this journey.

To my sister, Allison Sprowls Reilly. Thank you for pushing me from the day I was born. Growing up it may not have seemed like it, but as I write this I am so grateful and lucky to have had you as a sister. I am so proud of the woman you have grown into. You are an awesome radiation therapist and your patients are lucky to have you. I hope that in the future you will eventually see the results from my work in the clinic, which is the true measure of a scientist. Never lose your sense of excitement and adventure. I wish that I was able to be a free-spirited as you are! You have work so hard to get to where you are today and it shows. Keep fighting and never settle for anything less than the best. Thank you for being my first friend and an amazing big sister, I love you. 
To my brother, Chris Mikolowsky. I will forever be indebted to you. You have been my mentor, my best friend, and my biggest supporter my entire life. When I envision myself in the future, I hope to become even half the father and man you are. I hope that I can make an impact on everyone's life even a fraction of the size of the one you have left on mine. Having you in front of me to watch grow and learn from wasn't always easy, but I would not have ever changed it. From helping me decide which college to choose from, to completely ignoring that advice, to helping me get a job with a worthwhile pay, to looking out for my financially well-being, for providing me with a place to live, a person to confide in, for helping to grow into the man I am today, and so much more I am eternally grateful. For as long as I can remember, you are the person I have wanted to be when I grow up. I hope that I have made you proud. You are the single greatest role model I could have asked for. These words alone do not describe how thankful I am to have had you as my big brother. I love you.

To my mother and father, Jackie and Sam Sprowls. Thank you for literally everything. You have provided me with my world, a home, a shelter from harm, a listening ear, endless encouragement, a shoulder to cry on, and endless love and support. Without you I would certainly not be where I am today. Because of you, I know that I can do anything I put my mind to. Even though I am sure it was tough, you raised me without fear. From early on in my life, and though I do not remember the earliest years, you raised me to chase after my dreams despite having an immune deficiency. You helped me to learn that a diagnosis did not 
define me and that I was just an ordinary boy with a dream. Thank you both for all that you have done in my life. I love you.

One last bit for anyone reading this. From the moment I saw this quote on a coffee mug it changed my perspective and the way I pursue everything in life. Whenever I experience doubt or am having a particularly rough go at it, I always remember this. I hope that you may find it as inspiring as I have.

"Go confidently in the direction of your dreams! Live the life you've imagined."

-Henry David Thoreau 
TABLE OF CONTENTS

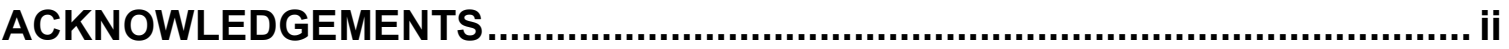

LIST OF FIGURES

LIST OF TABLES …............................................................................... xviiii

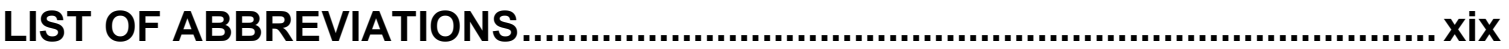

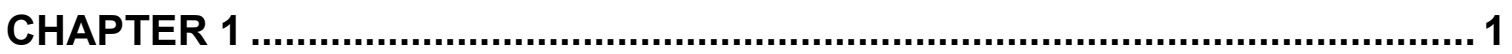

INTRODUCTION

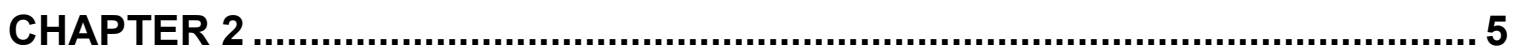

Improving CNS Delivery to Brain Metastases by Blood-Tumor Barrier

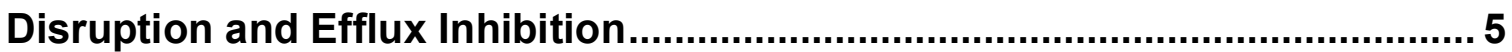

2.1 Brain Metastases and Treatment Failure …........................................... 5

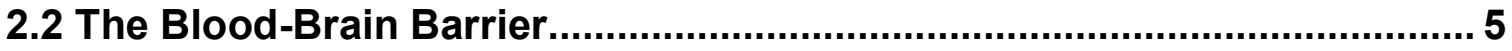

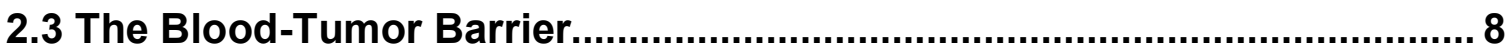

2.4 BBB/BTB Disruption for Increased Therapeutic Potential .....................11

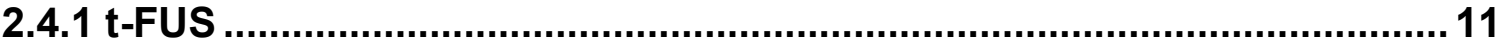

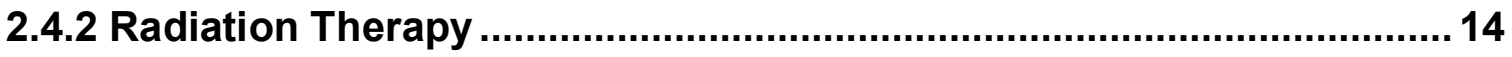

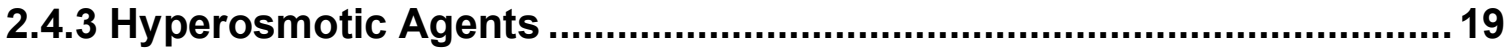

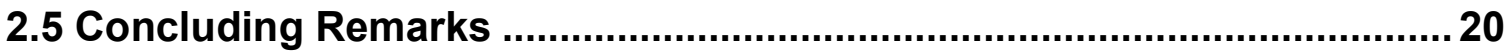

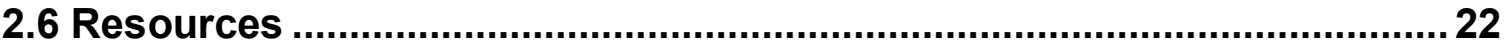

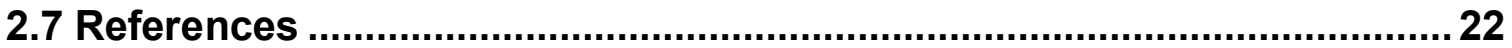

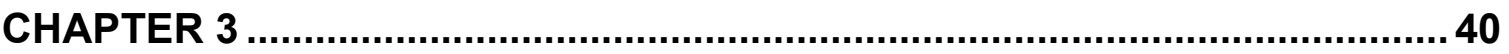


The Hypomethylating Agent Azacitidine is Effecive in Treating Brain Metastasis of Triple Negative Breast Cancer Through Regulation of DNA Methylation of the Keratine 18 Gene ........................................................... 40

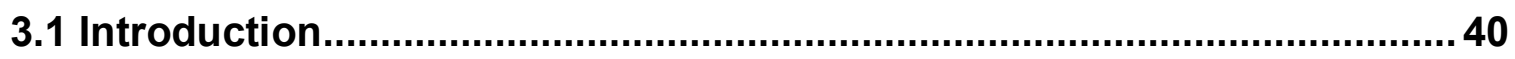

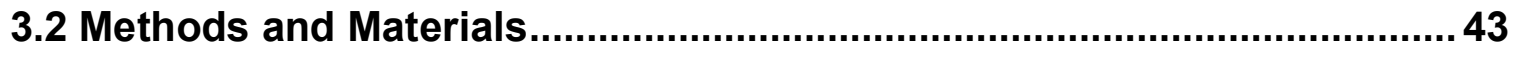

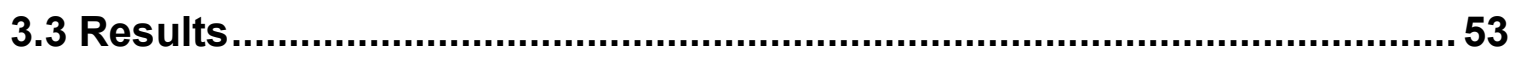

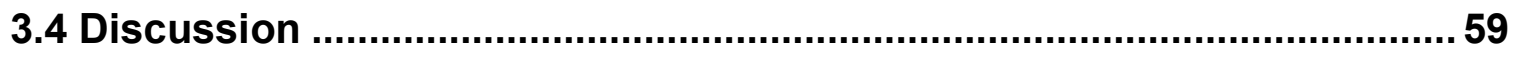

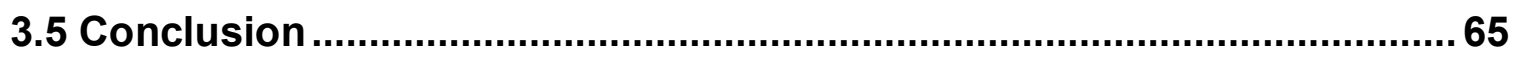

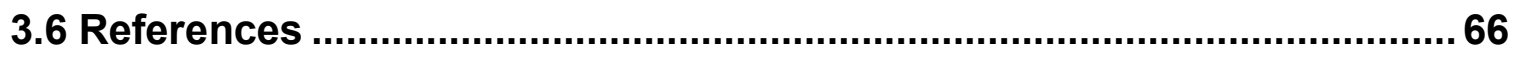

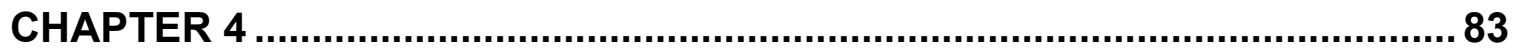

Cannabidiol Inhibits P-glycoprotein Mediated Efflux at the Blood-Brain

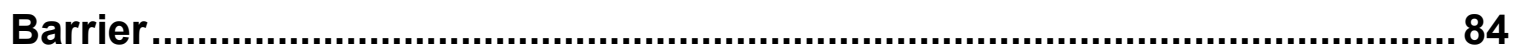

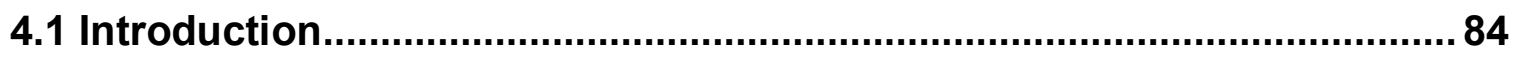

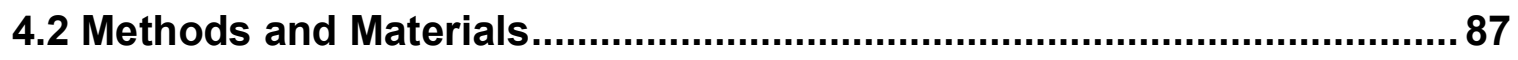

4.3 Results

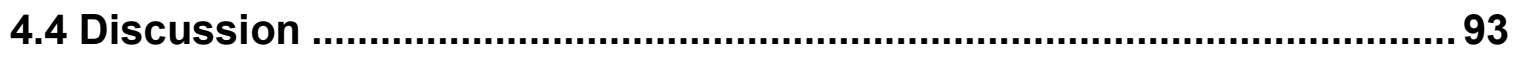

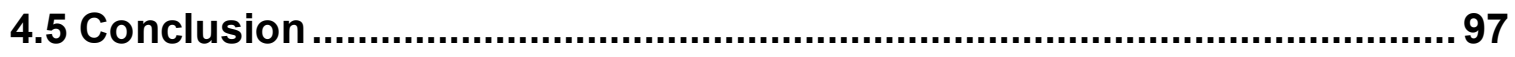

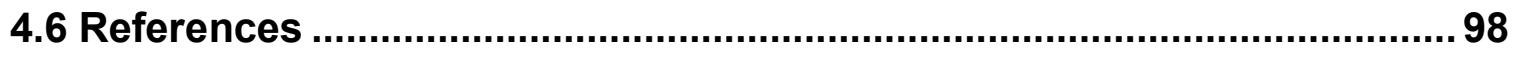

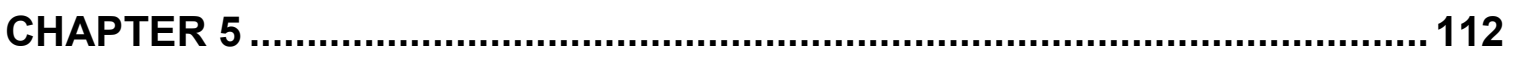

Radiation Increases BTB Permeability in a Preclinical Model of Breast

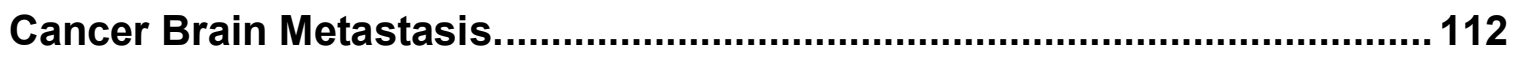

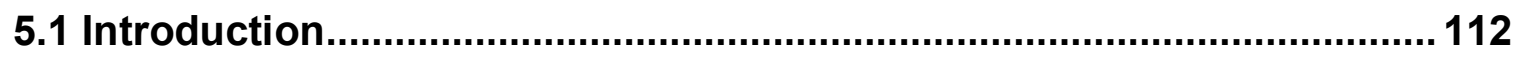

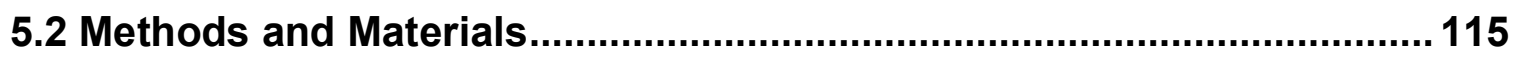




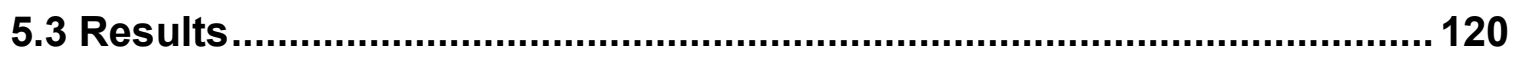

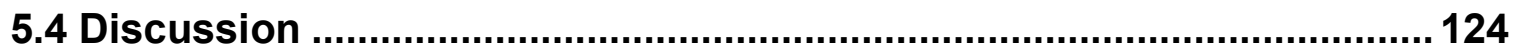

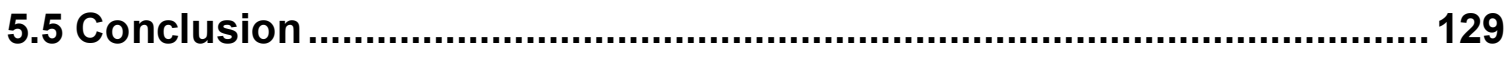

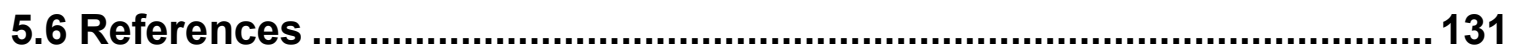

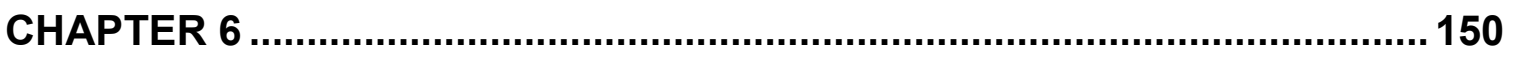

A Review of Mathematics Determing Solute Uptake at the Blood-Brain

Barrier in Normal and Pathological Conditions....................................... 151

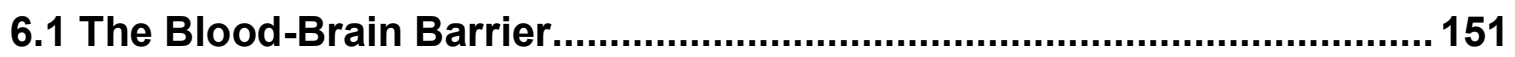

6.2 Mapping Drug Kinetics at the Blood-Brain Barrier...............................152

6.3 Active Efflux at the Blood-Brain Barrier. .............................................. 155

6.4 Flow- vs. Perfusion-Limited Blood-Brain Barrier Transport................. 158

6.5 Preclinical Measurements of Blood-Brain Barrier Permeability in

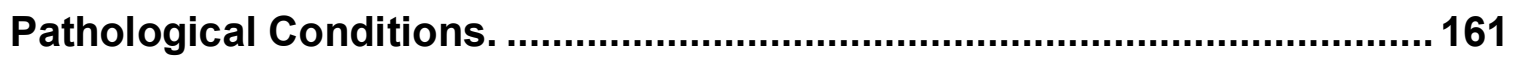

6.6 Clinical Blood-Brain Barrier PK in Disease States and Preclinical Model

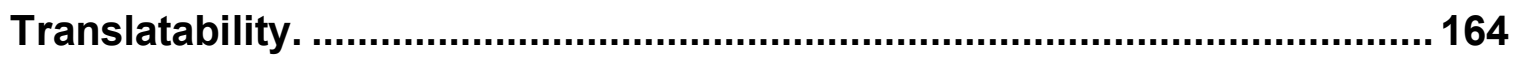

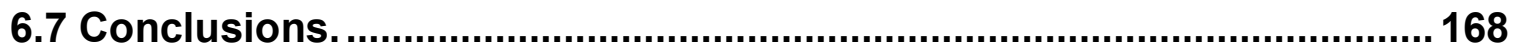

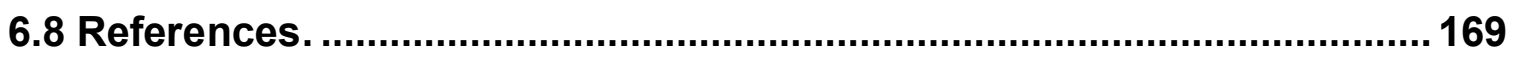

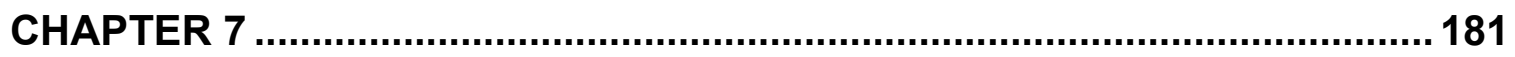

Conclusion and Future Directions. ........................................................ 181

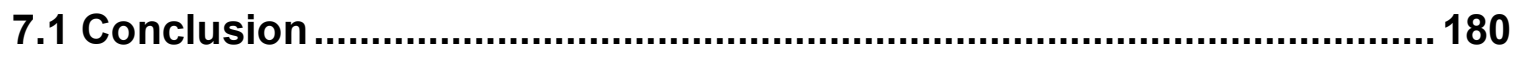

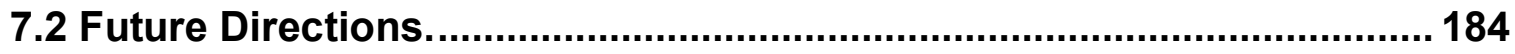




\section{LIST OF FIGURES}

Figure 2.1. Anatomy and physiology of the blood-brain barrier.

Figure 2.2. The blood-tumor barrier has altered anatomy and physiology. 38

Figure 2.3. Techniques for disruption of the blood-brain barrier. 39

Figure 3.1. Brain colonizing breast cancer cells $(231 \mathrm{Br})$ have a different oncological penotype compared to parental breast cancer 231 cells

Figure 3.2. 231Br breast cancer cells are more sensitize to AA treatent

compared to 231 cells.

Figure 3.3. AZA differentially inhibits Wnt signaling transduction pathway and angiogenesis related markers in vitro.

Figure 3.4. 231 $\mathrm{Br}$ cells have higher migration and invasion potential compared to 231 cells.

Figure 3.5. The keratin 18 gene is hypermethylated in brain colonizing cells compared to regular breast cancer cells. 80

Figure 3.6. AZA decreases tumor burden and improves survival in mice with brain metastasis of breast cancer. 82

Figure 4.1. Cannabidiol has anticancer activity in the brain tropic MDA-MB231Br breast cancer cell line and inhibits P-gp at the in-vivo blood-brain barrier. 106 
Figure 4.2. Cannabidiol prevents brain metastasis associated weight loss and reduces tumor burden in a preclinical brain metastasis prevention

model. 108

Figure 4.3. Cannabidiol in combination with paclitaxel has little effect on tumor burden and overal survival compared to vehicle or monotherapy treatments.

Figure 5.1. Calibratio curve at isocenter generated using WVU HSC's Xstrahl small animal irradiator (SAI).

Figure 5.2. Dose hoogeneity output of a $10 \times 10 \mathrm{~mm}$ field size irradiated to a target dose of 5.4Gy. 143

Figure 5.3. Histological verification of half-brain irradiation in an in vivo system. 143

Figure 5.4. The BBB remains intact in athymic Nu/Nu mice but is disrupted at an intermediate dose in immune competent FVB mice. 145 Figure 5.5. Permeability of metastatic brain lesions increases in a time and dose dependent manner following half-brain irradiation. 147

Figure 6.1 Anatomical differences between (A) blood-brain barrier vasculature and $(B)$ disrupted vasculature. 177

Figure 6.2. Correlation of solute BBB permeability, indicated by its permeability surface area (PS) product, with the $\log (P \div \sqrt{M W})$. 178 
Figure 6.3. A schematic representation of (A) extraction-limited and (B) flowlimited solute transfer across the BBB. ...........................................179

Figure 6.4. Differential tracer uptake in various imaging modalities.......... 180 


\section{LIST OF TABLES}

Table 2.1 BBB/BTB Disruption Techiques in Ongoing or Completed Clinical

Trials. 36

Table 4.1. Cannabidiol half-maximal inhibitory concentration in various cancer cell lines

Table 5.1. Comparisonof dose, BED, and permeability changes among literature reports investigating the BBB and radiation therapy in preclinical models.

Table 5.2. Comparisonof dose, BED, and permeability changes among literature reports investigating the BBB and radiation therapy in clinical patients. 


\section{LIST OF ABBREVIATIONS}

AZA: azacitidine

BAD: Bcl-2-associated death promoter

BAX: Bcl-2-associated X

BBB: blood-brain barrier

BCBM: breast cancer brain metastasis

BCRP: breast cancer resistance protein

BED: biological effective dose

BLI: bioluminescent imaging

BTB: blood-tumor barrier

CBD: cannabidiol

DNMT: DNA methyltransferase

EMT: epithelial-mesenchymal transition

ER: estrogen receptor

FDA: Food and Drug Administration

GBM: glioblastoma multiforme

GSK-3: glycogen synthase kinase-3

HER2: human epidermal growth factor receptor 2

HIF: hypoxia-inducible factor; MDS: myelodysplastic syndromes

MMP: matrix metalloproteinase

MRP1: multidrug resistance protein 1

MTT: 3-(4,5-Dimethylthiazol-2-yl)-2,5-diphenyltetrazolium bromidefor OD: optical density 
P-gp: P-glycoprotein

PR: progesterone receptor

PBS: phosphate-buffered saline

PTX: paclitaxel

RBC: red blood cel

SAI: small animal irradiator

SRS: stereotactic radiosurgery

TGF: transforming growth factor

TNBC: triple negative breast cancer

TxRd: Texas red

VEGF: vascular endothelial growth factor

WBRT: whole-brain radiotherapy 


\section{Chapter 1}

\section{Introduction}

The blood-brain barrier (BBB) is a dynamic, physiochemical barrier at the interface between the systemic circulation and the brain. In health, the BBB acts as a mechanism for neuroprotection, nutrient exchange, and maintenance of cerebral blood flow among other various functions. In many disease states, such as ischemic stroke, Alzheimer's, systemic lupus, cancer, etc., the BBB can be disrupted, or "leaky".

In metastatic brain tumors, the BBB, or the blood-tumor barrier (BTB), is heterogeneously disrupted. The foundational work from our lab described that while the BTB is leaky, a majority of brain lesions never reach cytotoxic concentrations of the various chemotherapies in a preclinical model of breast cancer brain metastases. Because of the BTB, most chemotherapeutics are near useless for brain tumors, partially due to either increased active efflux or poor drug permeation.

Cancer metastasis is defined as the spread of a primary cancer, i.e. breast cancer, lung cancer, or melanoma, to a secondary site, such as the lungs or brain. Metastasis to the brain is estimated to occur in up to $10 \%$ of all cancer. Specifically, for breast cancer patients, up to $30 \%$ of the women unfortunately diagnosed with primary breast cancer are at risk for development of brain 
metastases. Survival for these women is poor and most patients typically survive less than two years from central nervous system disease onset. Treatment options are few, and mostly palliative, for brain tumors. These modalities include radiation therapy, either whole-brain radiotherapy or stereotactic radiosurgery, surgical resection, and/or systemic chemotherapy. As mentioned, these options are not curative, but serve to provide the hopes of a few more months of life.

For the aforementioned details and various other possible neurological complications, novel treatment strategies to manage breast cancer brain metastases are crucial. In the past few decades, novel chemotherapeutics, redesigned drug formulations, and alternate routes of drug administration have provided hope, but have failed to provide modest survival benefits. In this dissertation, we investigate the use of an FDA approved hypomethylating agent in the treatment of breast cancer brain metastasis (BCBM). Additionally, we describe the ability of cannabidiol to inhibit P-glycoprotein mediated efflux at the BBB and evaluate the efficacy of combinatorial therapy utilizing cannabidiol in our preclinical model of BCBM. Lastly, we describe the effects of radiation therapy on the BBB and the BTB, regarding the time frame of disruption posttherapy and the impact said disruption may have on chemotherapy permeability.

Chapter 2 provides an in-depth review of the BBB, brain metastases, active efflux at the BBB, and novel treatment modalities used to physically disrupt the BBB for 
increased chemotherapeutic efficacy when treating brain tumors. Clinical trials utilizing these techniques are also discussed herein.

Chapter 3 details the use of azacitidine, an approved hypomethylating agent, in the treatment of brain metastases of breast cancer. In this chapter the potential mechanism of action of azacitidine is also explored in vitro.

Chapter 4 investigates the use of cannabidiol for increased drug distribution to brain through inhibition of P-glycoprotein efflux, as well as its efficacy in both the prevention and treatment of BCBM.

Chapter 5 describes the effect of development of a small animal radiation research platform using a host of dosimetry techniques. Additionally, this model was used to evaluate the effects of radiation therapy on the BBB and the BTB in vivo regarding permeability of the fluorescent tracer, Texas Red. A comparison of literature reported experiments involving radiation and the BBB or BTB is provided.

Chapter 7 reviews the classical pharmacokinetics regarding solute uptake at the BBB. Mathematics for determining the rate at which a compound is effluxed from the brain, Kout, are also described. When applied to disease models these mathematics provide quantitative measurements of drug or tracer uptake and provide more analytical data than those reported in fold-change. These 
mathematics will provide a framework for future work investigating tracer uptake following radiation.

In summary, this dissertation provides a detailed discussion of the blood-brain and blood-tumor barriers, and describes both physical and pharmacological means to treat $\mathrm{BCBM}$. When viewed together, these data highlight various means to bypass brain barriers through rational drug design, active efflux inhibition, and physical disruption. Additionally, a preclinical model for radiation research was also established at our university. Lastly, this dissertation provides a mathematical approach for quantification of solute uptake in normal and compromised brain. No matter the experiment, the main goal of the work herein was to improve upon the use of currently available treatments to provide even modest survival benefits for those suffering from BCBM. 


\section{Chapter 2}

\section{Improving CNS delivery to brain metastases by Blood Tumor Barrier disruption.}

\subsection{Brain Metastases and Treatment Failure}

Brain metastasis is an overwhelming morbidity of late stage cancer progression. Central nervous system (CNS) metastases occur in approximately $10 \%$ of all cancer types [1]. Recent increases in brain metastases are thought to be caused by improved control of systemic disease and increasingly sensitive imaging modalities [2]. Patients with CNS disease typically succumb within two years of diagnosis [3-5]. Therapies for brain lesions are mostly palliative, and rarely ever curative. These therapies include bulk surgical resection of the tumor(s), radiation therapy (either whole-brain and/or stereotactic), and/or systemic chemotherapy [6]. The blood-brain barrier (BBB), the brain's innate defense system against blood delivered harmful substances, prevents delivery of most all efficacious systemic chemotherapies into brain tissue [7].

\subsection{The Blood-brain Barrier}

The BBB's unique properties occur as a result of specific interactions between endothelial cells, pericytes, astrocytes, microglia and neurons, and their molecular components as seen in Figs. 2.1, 2.3a [8]. Proper regulation and 
function of the BBB is dependent on uninhibited interaction and communication between these cells.

\section{Endothelial Cells}

Brain microvascular endothelial cells (EC) form the foundation layer of the BBB and are crucial to the maintenance of its integrity. The ECs of the BBB are polarized in structure, as their luminal and abluminal surfaces have diverse biochemical and functional features; e.g. increased luminal $\gamma$-glutamyl transpeptidase expression [9]. The specialized BBB ECs have a high degree of expression of various transporters, including P-glycoprotein (P-gp, ABCB1), breast cancer resistance protein (BCRP, $A B C G 2)$, multi drug resistance protein, and various nutrient transporters [9]. These transporters move nutrients into the brain and efflux waste and other molecules out of the brain. Efflux pump expression is a major obstacle in overcoming drug delivery to the brain.

One of the most crucial features of ECs is their expression of tight junctions (TJ), which stabilizes the integrity of the BBB. The expression of TJ's is induced by pericytes and results in a non-fenestrated vasculature preventing any unwanted "leaking" of luminal contents into the parenchyma of the brain. The TJ proteins are comprised of various transmembrane proteins including claudins, occludins, junctional adhesion molecules and accessory proteins, such ZO-1 and ZO-2 [10]. Another important trafficking molecule in normal BBB anatomy is major facilitator superfamily domain $2 a$ (Mfsd2a). This protein is important for development of a functional BBB and is required for movement of docosahexaenoic acid into brain 
tissues [11, 12]. Importance of this transporter in BBB integrity and functionality is demonstrated by mice with genetically removed Mfsd2a that have decreased docosahexaenoic acid transport and increased disruption of the vascular barrier in brain [13].

\section{Pericytes}

Pericytes share the basement membrane with ECs and attach to them by 'pegsocket' junctions within the cerebral vasculature [14, 15]. During developmental stages and adult life, pericytes are recruited to EC of the BBB through several signaling methods, primarily the platelet derived growth factor- $\beta$ pathway [14].

The presence of pericytes is critical for proper BBB function and development. These accessory cells directly influence permeability of the BBB by inducing EC TJ formation [16]. Next, pericytes regulate cerebral blood flow and waste clearance, disruption of which is associated with multiple brain pathologies, such as Alzheimer's [15, 16]. Pericytes are shown to polarize astrocytic end-foot processes surrounding the $\mathrm{BBB}$, and further are shown to regulate $\mathrm{EC}$ gene expression, increasing their viability through the Bcl2I2 pathway $[17,18]$.

\section{Astrocytes}

Astrocytic end-feet processes surround the BBB almost entirely. Their end-feet connect to the basement membrane through junctional molecules, including dystroglycan as well as channels like aquaporin 4 , a molecule shown to maintain 
water homeostasis in the brain [10,19]. Astrocytes play several roles in the regulation of the BBB. They assist in regulation of cerebral blood flow through $\mathrm{Ca}^{2+}$ signaling following neuronal perturbation [20]. Further, astrocytes are responsible for maintenance and formation of EC TJ. Sonic hedgehog, ang-1, and transforming growth factor signaling pathways influence this maintenance $[10,21]$. Lastly, astrocytes directly impact vascular growth and proliferation through ang-1 and vascular endothelial growth factor (VEGF) secretion [10, 22, 23].

Microglia

Microglia are the resident immune cells of the brain. These cells play a role in both pro- and anti-inflammatory responses. Depending on their pro-inflammatory (M1) or anti-inflammatory (M2) phenotypes, they control inflammation through release of various molecular cytokines. Microglia are involved in angiogenesis, especially near EC tip cells, suggesting their influence in cerebral vascular development $[24,25]$. However their role in maintenance of the integrity of a healthy BBB is still unknown.

\subsection{The Blood-tumor Barrier}

Of the primary cancers that migrate to the brain, lung, breast, melanoma, and renal cancers comprise the majority of metastatic brain tumors affecting $\sim 50 \%$, $\sim 15 \%, \sim 10 \%$, and $\sim 5 \%$ of patients respectively [2]. Brain metastasis occurs when a circulating tumor cell, of a primary systemic tumor (i.e., breast, lung, 
melanoma, renal), detaches from the initial tumor mass and arrests in the brain microvascular capillary network, extravasates through the vessel wall into the perivascular space, and survives and proliferates into a new lesion [26, 27]. From initial metastatic colonization, the newly "seeded" brain metastatic tumor cells coopt the brain vasculature eliciting neo-angiogenesis and microenvironment remodeling to promote tumor growth and further invasion. The newly formed neurovascular-tumor unit is termed the blood-tumor barrier (BTB, Fig. 2.2) and has differential properties concerning therapy pharmacokinetics and action in comparison to the intact BBB.

The BTB is inherently "leaky", lacking tight junctions and astrocytic-endothelial contacts resulting in significant heterogeneous permeability from lesion to lesion within the brain $[28,29]$. As lesions continue to outgrow their oxygen supply, angiogenesis occurs driven largely by VEGF. These new vessels are inherently leaky compared to the BBB phenotype. Dynamic angiogenesis during metastatic progression is different among brain lesions, which is thought to contribute to the heterogeneity in tumor permeability to chemotherapy. Additional contributions to increased permeability of the BTB include the lack of physiological TJ protein expression causing fenestra and discontinuous endothelia [28, 30]. Inconsistencies of junctional protein expression can allow for the passive permeability of cytotoxic therapies into tumor tissue. Interestingly mfsd2a is down-regulated at the BTB and promotes brain metastatic outgrowth due to lack 
of astrocytes promoting endothelial expression of $\mathrm{mfsd} 2 \mathrm{a}$, further contributing to BBB leakage in brain tumors [31].

Efflux mediated by P-gp (ABCB1) and BCRP (ABCG2) at the BBB limits distribution to normal brain of most chemotherapeutic agents. In the BTB setting, P-gp and BCRP have been found to be increased at the luminal membrane, as well as in the plasma membrane of tumor cells [32-34]. In preclinical mouse models, Elmquist and colleagues have demonstrated the active efflux of a host of agents used to treat melanoma and lung cancer brain metastases [32, 35, 36].

Other cellular and molecular properties of the BTB are prompted by astrocytes, pericytes and microglia. Astrocytes function to support and protect neuronal cells from damage and apoptosis through secretion of inflammatory cytokines such as TNFa, IL1, and IL6. Release of these cytokines encourages tumor proliferation and survival.[37] Additionally, astrocytes release exosomes containing miRNA19a, which serves to induce loss of PTEN and promote further outgrowth and invasion of tumor cells within the brain [37, 38]. Microglia in the brain tumor microenvironment are known to secrete multiple growth factors and cytokines, such as TGF $\beta$, TNFa, IL1, IL6, VEGF, EGF, and many metalloproteinases [39]. The molecular entities secreted by microglia promote tumor proliferation and invasion, as well as support angiogenesis [39]. Microglia cell populations also support colonization through the Wnt pathway, an effect attenuated with addition of Wnt inhibitors [40]. Pericyte subpopulations are known to contribute to BBB 
integrity and therefore permeability. Desmin+ pericytes are found in high concentrations in brain metastases and their presence is associated with high permeability [41].

Taken together, the distinct physical and molecular impedance the BTB plays in cancer treatment may seem insurmountable. In fact, the BTB, even in the presence of heterogeneous disruption, limits drug accumulation to the degree that there is limited apoptosis and cytotoxicity in nearly $90 \%$ of metastatic lesions in experiments utilizing preclinical models of breast cancer brain metastasis [4247]. Inability of drugs to distribute to brain tumor tissues has led to the progression of techniques aimed at disrupting the BBB.

\subsection{BBB/BTB Disruption for Increased Therapeutic Potential.}

Disruptive CNS barrier techniques have increasingly become a research focus. Three highly investigated areas include the use of focused transcranial ultrasound (t-FUS) coupled with intravenously delivered microbubbles, hyperosmotic agents, and to a lesser degree radiation therapy that elicits transient changes in BBB permeability. Each of these applied to the treatment of metastatic brain lesions may lead to increased drug distribution and improve efficacy of many approved therapeutics. A list of ongoing or completed clinical trials utilizing disruption techniques can be found in Table 1.

\subsubsection{Focused Transcranial Ultrasound}


Transient focused transcranial ultrasound (t-FUS) with concurrent administered intravenous microbubbles has been investigated as it can increase barrier permeability and improve distribution of CNS targeted therapeutics. Preliminary studies on mechanisms of BBB disruption indicate that the minimally invasive low intensity t-FUS coupled with the acoustic cavitation produced by the microbubbles cause molecular changes in tight junctions through decreased expression of claudin-5, ZO-1 and occludin, which enable the paracellular transport of genomic and chemical therapeutics as well as initiate inflammatory responses associated with damage-associated molecular patterns (Fig. 2.3b) $[48,49]$. Combined with the higher hydraulic conductivity of interstitial fluid to the solid tumors, these changes have been used not only for higher tumor targeted delivery of many small molecule therapeutics but also for genes and immune cells [50-52].

Ultrasound influences the rate and extent of microbubble cavitation through its physicochemical properties that may lead to the production of stable or inertial cavitations. Under the influence of the FUS, microbubbles can undergo harmonic or non-harmonic oscillations which are responsible for the transient tight junction disruption; or undergo expansion and eventual collapse which can result in supplemental leakage or permanent damage $[53,54]$. The amplitude and frequency of the ultrasound govern the mechanical index of the microbubbles and lead to enhanced disruption by specialized mechanisms including the pushpull action mediated broadening of ECs, high shear stress through micro-stream 
production, acoustic radiation, and pressure gradient mediated microbubble displacement $[53,55]$. However, when microbubbles undergo unstable expansions and collapse it can lead to high EC lining pressure which may cause fragmentation of microbubbles resulting in mircro-jets and shock-waves. Additionally, microbubbles may also undergo free radical formation depending on microbubble lipid content and the degree of cell membrane permeabilization [56]. Altering the parameters of microbubbles enables their use as drug delivery devices as shown by a recent study that used a novel nitrogen based folate conjugated microbubble system encapsulated with methotrexate to increase its site-specific delivery and thus drug efficacy using high intensity focused ultrasound [57].

A recent study investigated the BBB/BTB penetration and cellular uptake of small (Doxorubicin) and large (ado-trastuzumab emtansine) molecules for an orthotopic brain metastasis of HER2 positive breast cancer model [50]. The study demonstrated that the small hydrophobic molecule showed a much higher (7fold) concentration in the extravascular compartment along with high tumor penetration when FUS was used as opposed to control. In contrast, despite showing a 2-fold increase in the extravasation and slightly higher tumor penetration, the long (4-6d) drug circulation and transient effect of ultrasound diminished the overall effect when compared to control on day 5. Another study investigated the antitumor efficacy of polymeric polysorbate 80 modified paclitaxel nanoparticles and found an increase in the median survival of U87-Luc 
glioma-bearing mice to 37 days when to the control's 26 days [58]. They demonstrated that the ultrasound mediated reduction in P-gp expression and tight junction disruption as well as apolipoprotein mediated endocytosis was responsible for the enhanced permeation of the nanoparticles. These pre-clinical studies in animal models have shown high efficacy leading to multiple trials to test the use of ultrasound in drug delivery for neurological diseases including Alzheimer's i, Parkinson's Disease ii with dementia and multiple gliomas iii.

Despite promising results, there are challenges such as high inertial cavitations of the microbubbles that cause vascular and tissue damage, reliance on expensive techniques like contrast magnetic resonance imaging to detect disruption, and lack of normalized experimental conditions. A study to reduce the inertial cavitation and provide an alternate treatment modality used closed loop cavitation mechanism to accurately provide $274.3 \mathrm{kHz}$ of ultrasound; increased both survival and tumor regression by increased doxorubicin delivery in glioma bearing rats [59]. An alternate semiautomatic approach to deliver the ultrasound used unfocused ultrasound devices implanted in patients with glioblastoma. The study correlated local acoustic brain pressures with signal enhancement of greater than 10 percent observed through ultrasound which was more in gray matter iv.

\subsubsection{Radiation Therapy}


The effects of radiation therapy on the BBB have been studied since the early 1980s [60]. However, the precedent of radiation therapy with subsequently timed chemotherapy was first suggested in 2002 by van Vulpen et. al [61]. The dose dependent response and time course of disruption of the BBB following radiation therapy is highly debated with the existence of contradictory reports. The pathophysiological changes following BBB disruption induced by radiation have been segregated into two main categories, acute and late phases [62-64]. Acute effects are thought to occur within the first 24 hours following cranial irradiation and, late effects are those described thereafter [65].

Mechanisms of radiation induced permeability (Fig. 2.3c) during the early stages after therapy include EC death and an increase in neuro-inflammation. Microvascular cell density and tight junction protein, ZO-1, expression was shown to decrease from 1 to 180 days following a single $10 \mathrm{~Gy}$ whole brain radiotherapy dose [66]. A similar study reported EC density decreases at a single 10Gy dose are greatest at 10 days following radiation therapy [67]. Another study indicating the death of ECs as an early event following cranial radiation observed an increase in apoptotic ECs peaking at 12 hours after radiotherapy at doses ranging from 5Gy to $100 \mathrm{~Gy}$ [68]. From these data, it appears evident that changes at the endothelial level occur, but the exact timing and mechanism are not clear. 
The neuro-inflammatory response following radiation insult is characterized by activation of astrocytes, microglia, ECs, and their inflammatory mediators. Astrocytic and microglia activation following cranial exposure to radiation have been indicated as early as 4 hours and as late as 6 months following radiation treatments demonstrated by increased GFAP and CD11b staining $[69,70]$. While these indicators of cellular activation are present, a number of cytokines and adhesion molecules are also variably increased following radiotherapy. In studies by Hong et al. and Kyrkanides et al. at four hours post radiation treatment, increases in CNS levels of TNF $\alpha$, IL-1 $\beta$, and IL-6 were shown $[71,72]$. In a similar study, Ruimeng et al. demonstrated the capacity for radiation therapy, at a dose of 50Gy, to increase immune cell activation and a panel of cytokines, including TNF- $\alpha$ and IL-6, at 12-weeks post treatment [73]. These research data suggest a critical role of the neuro-inflammatory response to radiation.

Taken together, the physiological responses to radiation alter the BBB/BTB in a manner which increases permeability. Data on the time course of increased permeability have been reported, but are variable among studies. Wilson et al. reported significantly altered permeability at 24 and 48 hours following cranial irradiation with a single dose of $20 \mathrm{~Gy}$, which could be rescued with anti TNF- $\alpha$ treatment [63]. Confirming this, a study of the rat BBB saw significant increases in permeability peaking at 24 hours post-therapy at a single dose of $20 \mathrm{~Gy}$ to $4.4-$, 10-, 38.2-, and 70-kDa FITC-dextran molecules [74]. Interestingly in Yuan et al.'s study, the time dependent increase in BBB permeability correlated well with an 
increase number of rolling leukocytes at the BBB, suggesting an increase in ICAM-1, a molecule expressed on the luminal surface of the BBB to aid in leukocyte trafficking to the brain parychema during an immune response [74]. Another study confirming early BBB disruption as soon as 24 hours following irradiation with single doses of 20 and 40Gy [75]. Each of these studies used a different means of irradiation, resulting in a specific dose rate for each respective study. This may provide information regarding the effect of dose rate on permeability related outcomes.

Another factor potentially contributing to permeability of the BBB/BTB may be fractionation schemes. Using daily doses of $4 \mathrm{~Gy}$ for 3 consecutive days, Crowe et al. demonstrated enhanced permeability of irradiated tumors at 24 hours posttreatment compared to their contralateral sham treated counterparts when analyzed using DCE MRI [76]. Fractionation may elicit potentially altered permeability outcomes. Additionally, the particular mode of irradiation may play a role in pathophysiologic response to irradiation as well. When comparing broad beam radiation to micro-beam radiation therapy, Bouchet et all showed higher permeability increases in tumors treated with microbeam radiation therapy compared to those treated with conventional broadbeam radiotherapy at all time points, with a maximum at 7 days following radiation treatment [77]. Of note, there was increased permeability in lesions treated with BBRT compared to nontreated regions [77]. 
These studies all provide insight as to when the permeability changes may occur following radiation treatment. Contrary to this work another study by Murrell et al. noted that a dose of 20Gy in 2 fractions was not able to increase tumor permeability in a preclinical model of breast cancer brain metastasis [63]. Their work was subjected to only two time points however, one week and 11 days post radiation treatments. It is important to note that both authors may be suggesting the correct response. BBB/BTB opening following radiation therapy treatment may be transient or biphasic in nature, with points of high and low permeability in different phases, similar to that of stroke pathology [78].

Clinically there is evidence of breakdown of the BBB and BTB after radiotherapy as well. In a study of 30 patients receiving WBRT or SRS, with 64 analyzed metastatic lesions, radiotherapeutic treatments improved the permeability of initial low leaky tumors at 2 weeks and 1 month post therapy [79]. However, there was little or decreased permeability in initially very leaky metastases [79]. Zeng and colleagues also showed that in NSCLC patients treated with WBRT and concurrent gefitinib therapy, increased drug penetration was observed in accordance with escalation of radiation dose [80]. Lim et al. saw increased gadolinium deposition in peri-tumoral areas in 44 glioblastoma patients, but no change in untreated areas, indicating BBB/BTB disruption following radiation therapy [81]. These date provide evidence for increased permeability following radiation, but none give information elucidating the time course or magnitude of increased permeability. 


\subsubsection{Hyperosmotic Agents}

Pre-clinical and clinical strategies have targeted the transient loosening or disruption of the BBB to increase permeability of therapeutics by techniques such as ultrasound, radiation or hyperosmotic agents like mannitol. One of the earliest techniques to disrupt the BBB using hyperosmotic agents was described by Neuwelt et al; wherein hyperosmotic mannitol administered via an intra-carotid injection was used to reversibly disrupt the BBB in canines [82]. The work demonstrated that when methotrexate was administered after the hyperosmotic agent, the drug levels were significantly higher (nearly 5-9 times as compared to control) in the ipsilateral cerebral hemisphere and contralateral hemisphere [82]. Although subsequent studies have failed to identify a singular mechanism underlying the mannitol mediated disruption, multiple distinct phenomena have been proposed. The most widely accepted theory of BBB opening is dehydration of the ECs followed by vasodilation induced shrinkage or contraction of the cells due to altered intracellular calcium levels (Fig. 2.3d) [83]. The resulting tension along with the calcium dependent actin and cadherin interaction leads to the widening of the tight junctions by increased bulk flow and solute diffusion. Other factors like nitric oxide, inflammatory mediators, bradykinin and mannitol induced tyrosine phosphorylation of $\mathrm{Axl}$ and beta-catenin have been implicated to augment the BBB disruption; however the exact mechanism is still not understood $[83,84]$. 
Despite facing early challenges like potential neurotoxicity, osmotic disruption has been successfully used in pre-clinical models for improving drug therapy. Pharmacological agents such as oligonucleotides that have poor brain delivery have improved distribution by hyperosmotic mannitol mediated BBB disruption [85]. The study further demonstrated a high dissemination of the oligonucleotide in the ipsilateral brain regions including the striatum, somatosensory cortex and thalamus upon co-administration of $25 \%$ mannitol and the oligonucleotide which was modified with a hydrophobic moiety. In addition, the striatum, thalamus, motor cortex, hippocampus and somatosensory cortex showed Huntington gene mRNA silencing even a week after the initial therapy administration.

\subsection{Concluding Remarks}

Disruption of the BBB/BTB by ultrasound, radiation or hyperosmotic agents appears to be a promising aid to the delivery of chemotherapy for brain metastases. Studies using these disruptive techniques have shown to have an auxiliary impact on the brain distribution of traditional therapy. However many questions still remain unanswered like the length and extent of its effect, translation to the clinic, cost to benefit and many more (see outstanding questions). Still, these disruptive techniques in combination with chemotherapy offer a unique system to combat the otherwise poor prognosis of brain metastases. 


\subsection{Resources}

i https://clinicaltrials.gov/ct2/show/NCT03671889

ii https://ClinicalTrials.gov/show/NCT03616860

iii https://ClinicalTrials.gov/show/NCT03608553

iv https://ClinicalTrials.gov/show/NCT02253212

v https://ClinicalTrials.gov/show/NCT02861898

vi https://ClinicalTrials.gov/show/NCT03714243

vii https://ClinicalTrials.gov/show/NCT03322813

viii https://ClinicalTrials.gov/show/NCT02343991

ix https://ClinicalTrials.gov/show/NCT03712293

x https://ClinicalTrials.gov/show/NCT03626896

xi https://ClinicalTrials.gov/show/NCT02031237

\subsection{References}

1. Shen, C.J., M. Lim, and L.R. Kleinberg, Controversies in the Therapy of Brain Metastases: Shifting Paradigms in an Era of Effective Systemic Therapy and LongerTerm Survivorship. Curr Treat Options Oncol, 2016. 17(9): p. 46.

2. Nayak, L., E.Q. Lee, and P.Y. Wen, Epidemiology of brain metastases. Curr Oncol Rep, 2012. 14(1): p. 48-54.

3. Witzel, I., et al., Breast cancer brain metastases: biology and new clinical perspectives. Breast Cancer Res, 2016. 18(1): p. 8.

4. Yousefi, M., et al., Lung cancer-associated brain metastasis: Molecular mechanisms and therapeutic options. Cell Oncol (Dordr), 2017. 40(5): p. 419-441. 
5. Glitza Oliva, I., H. Tawbi, and M.A. Davies, Melanoma Brain Metastases: Current Areas of Investigation and Future Directions. Cancer J, 2017. 23(1): p. 68-74.

6. Matzenauer, M., D. Vrana, and B. Melichar, Treatment of brain metastases. Biomed Pap Med Fac Univ Palacky Olomouc Czech Repub, 2016. 160(4): p. 484-490. 7. Abbott, N.J., Blood-brain barrier structure and function and the challenges for CNS drug delivery. J Inherit Metab Dis, 2013. 36(3): p. 437-49.

8. Chow, B.W. and C. Gu, The molecular constituents of the blood-brain barrier. Trends Neurosci, 2015. 38(10): p. 598-608.

9. Betz, A.L., J.A. Firth, and G.W. Goldstein, Polarity of the blood-brain barrier: distribution of enzymes between the luminal and antiluminal membranes of brain capillary endothelial cells. Brain Res, 1980. 192(1): p. 17-28.

10. Michinaga, S. and Y. Koyama, Dual Roles of Astrocyte-Derived Factors in Regulation of Blood-Brain Barrier Function after Brain Damage. Int J Mol Sci, 2019. 20(3).

11. Nguyen, L.N., et al., Mfsd2a is a transporter for the essential omega-3 fatty acid docosahexaenoic acid. Nature, 2014. 509(7501): p. 503-6.

12. Ben-Zvi, A., et al., Mfsd2a is critical for the formation and function of the bloodbrain barrier. Nature, 2014. 509(7501): p. 507-11.

13. Andreone, B.J., et al., Blood-Brain Barrier Permeability Is Regulated by Lipid Transport-Dependent Suppression of Caveolae-Mediated Transcytosis. Neuron, 2017. 94(3): p. 581-594 e5.

14. Rustenhoven, J., et al., Brain Pericytes As Mediators of Neuroinflammation. Trends Pharmacol Sci, 2017. 38(3): p. 291-304.

15. Zhao, Z., et al., Establishment and Dysfunction of the Blood-Brain Barrier. Cell, 2015. 163(5): p. 1064-1078. 
16. Daneman, R., et al., Pericytes are required for blood-brain barrier integrity during embryogenesis. Nature, 2010. 468(7323): p. 562-6.

17. Matsumoto, J., et al., Tumor necrosis factor-alpha-stimulated brain pericytes possess a unique cytokine and chemokine release profile and enhance microglial activation. Neurosci Lett, 2014. 578: p. 133-8.

18. Armulik, A., et al., Pericytes regulate the blood-brain barrier. Nature, 2010. 468(7323): p. 557-61.

19. Amtul, Z. and J.D. Hepburn, Protein markers of cerebrovascular disruption of neurovascular unit: immunohistochemical and imaging approaches. Rev Neurosci, 2014. 25(4): p. 481-507.

20. Manninen, T., R. Havela, and M.L. Linne, Computational Models for CalciumMediated Astrocyte Functions. Front Comput Neurosci, 2018. 12: p. 14.

21. Wang, Y., et al., Interleukin-1beta induces blood-brain barrier disruption by downregulating Sonic hedgehog in astrocytes. PLoS One, 2014. 9(10): p. e110024.

22. Meng, Z., et al., Ectopic expression of human angiopoietin-1 promotes functional recovery and neurogenesis after focal cerebral ischemia. Neuroscience, 2014. 267: p. $135-46$

23. Venkat, P., et al., Angiopoietin-1 Mimetic Peptide Promotes Neuroprotection after Stroke in Type 1 Diabetic Rats. Cell Transplant, 2018. 27(12): p. 1744-1752.

24. Sasaki, A., Microglia and brain macrophages: An update. Neuropathology, 2017. 37(5): p. 452-464.

25. Lee, E. and W.S. Chung, Glial Control of Synapse Number in Healthy and Diseased Brain. Front Cell Neurosci, 2019. 13: p. 42.

26. Ramakrishna, R. and R. Rostomily, Seed, soil, and beyond: The basic biology of brain metastasis. Surg Neurol Int, 2013. 4(Suppl 4): p. S256-64. 
27. Paget, S., The distribution of secondary growths in cancer of the breast. 1889. Cancer Metastasis Rev, 1989. 8(2): p. 98-101.

28. Lockman, P.R., et al., Heterogeneous blood-tumor barrier permeability determines drug efficacy in experimental brain metastases of breast cancer. Clin Cancer Res, 2010. 16(23): p. 5664-78.

29. Shah, N., et al., Investigational chemotherapy and novel pharmacokinetic mechanisms for the treatment of breast cancer brain metastases. Pharmacol Res, 2018. 132: p. 47-68.

30. Hendricks, B.K., A.A. Cohen-Gadol, and J.C. Miller, Novel delivery methods bypassing the blood-brain and blood-tumor barriers. Neurosurg Focus, 2015. 38(3): p. E10.

31. Tiwary, S., et al., Metastatic Brain Tumors Disrupt the Blood-Brain Barrier and Alter Lipid Metabolism by Inhibiting Expression of the Endothelial Cell Fatty Acid Transporter Mfsd2a. Sci Rep, 2018. 8(1): p. 8267.

32. Gampa, G., et al., Drug delivery to melanoma brain metastases: Can current challenges lead to new opportunities? Pharmacol Res, 2017. 123: p. 10-25.

33. Aryal, M., et al., Effects on P-Glycoprotein Expression after Blood-Brain Barrier Disruption Using Focused Ultrasound and Microbubbles. PLoS One, 2017. 12(1): p. e0166061.

34. Adkins, C.E., et al., P-glycoprotein mediated efflux limits substrate and drug uptake in a preclinical brain metastases of breast cancer model. Front Pharmacol, 2013. 4: p. 136.

35. Gampa, G., et al., Brain Distribution and Active Efflux of Three panRAF Inhibitors: Considerations in the Treatment of Melanoma Brain Metastases. J Pharmacol Exp Ther, 2019. 368(3): p. 446-461. 
36. Kim, M., et al., Brain distribution of a panel of EGFR inhibitors using cassettedosing in wild-type and Abcb1/Abcg2 deficient mice. Drug Metab Dispos, 2019.

37. Wasilewski, D., et al., Reactive Astrocytes in Brain Metastasis. Front Oncol, 2017. 7: p. 298.

38. Zhang, L., et al., Microenvironment-induced PTEN loss by exosomal microRNA primes brain metastasis outgrowth. Nature, 2015. 527(7576): p. 100-104.

39. Wu, S.Y. and K. Watabe, The roles of microglia/macrophages in tumor progression of brain cancer and metastatic disease. Front Biosci (Landmark Ed), 2017. 22: p. 1805-1829.

40. Pukrop, T., et al., Microglia promote colonization of brain tissue by breast cancer cells in a Wnt-dependent way. Glia, 2010. 58(12): p. 1477-89.

41. Lyle, L.T., et al., Alterations in Pericyte Subpopulations Are Associated with Elevated Blood-Tumor Barrier Permeability in Experimental Brain Metastasis of Breast Cancer. Clin Cancer Res, 2016. 22(21): p. 5287-5299.

42. Adkins, C.E., et al., Characterization of passive permeability at the blood-tumor barrier in five preclinical models of brain metastases of breast cancer. Clin Exp Metastasis, 2016. 33(4): p. 373-83.

43. Bohn, K.A., et al., Semi-automated rapid quantification of brain vessel density utilizing fluorescent microscopy. J Neurosci Methods, 2016. 270: p. 124-131.

44. Bohn, K.A., et al., Inhibition of VEGF and Angiopoietin-2 to Reduce Brain Metastases of Breast Cancer Burden. Front Pharmacol, 2017. 8: p. 193.

45. Mittapalli, R.K., et al., Quantitative Fluorescence Microscopy Measures Vascular Pore Size in Primary and Metastatic Brain Tumors. Cancer Res, 2017. 77(2): p. 238246. 
46. Mittapalli, R.K., et al., Quantitative fluorescence microscopy provides high resolution imaging of passive diffusion and P-gp mediated efflux at the in vivo bloodbrain barrier. J Neurosci Methods, 2013. 219(1): p. 188-95.

47. Terrell-Hall, T.B., et al., Trastuzumab distribution in an in-vivo and in-vitro model of brain metastases of breast cancer. Oncotarget, 2017. 8(48): p. 83734-83744.

48. Zhao, B., et al., Blood-brain barrier disruption induced by diagnostic ultrasound combined with microbubbles in mice. Oncotarget, 2018. 9(4): p. 4897-4914.

49. Kovacs, Z.I., et al., Disrupting the blood-brain barrier by focused ultrasound induces sterile inflammation. Proc Natl Acad Sci U S A, 2017. 114(1): p. E75-E84.

50. Arvanitis, C.D., et al., Mechanisms of enhanced drug delivery in brain metastases with focused ultrasound-induced blood-tumor barrier disruption. Proc Natl Acad Sci U S A, 2018. 115(37): p. E8717-E8726.

51. Mead, B.P., et al., Targeted gene transfer to the brain via the delivery of brainpenetrating DNA nanoparticles with focused ultrasound. J Control Release, 2016. 223: p. 109-117.

52. Curley, C.T., et al., Focused Ultrasound Immunotherapy for Central Nervous System Pathologies: Challenges and Opportunities. Theranostics, 2017. 7(15): p. 36083623.

53. Dasgupta, A., et al., Ultrasound-mediated drug delivery to the brain: principles, progress and prospects. Drug Discov Today Technol, 2016. 20: p. 41-48.

54. Jin, Q., et al., Inertial cavitation initiated by polytetrafluoroethylene nanoparticles under pulsed ultrasound stimulation. Ultrason Sonochem, 2016. 32: p. 1-7.

55. Chen, K.T., K.C. Wei, and H.L. Liu, Theranostic Strategy of Focused Ultrasound Induced Blood-Brain Barrier Opening for CNS Disease Treatment. Front Pharmacol, 2019. 10: p. 86. 
56. Jia, C., et al., Generation of Reactive Oxygen Species in Heterogeneously Sonoporated Cells by Microbubbles with Single-Pulse Ultrasound. Ultrasound Med Biol, 2018. 44(5): p. 1074-1085.

57. Lee, S., et al., Ultrasound-mediated drug delivery by gas bubbles generated from a chemical reaction. J Drug Target, 2018. 26(2): p. 172-181.

58. Li, Y., et al., Mechanisms of enhanced antiglioma efficacy of polysorbate 80modified paclitaxel-loaded PLGA nanoparticles by focused ultrasound. J Cell Mol Med, 2018. 22(9): p. 4171-4182.

59. Sun, T., et al., Closed-loop control of targeted ultrasound drug delivery across the blood-brain/tumor barriers in a rat glioma model. Proc Natl Acad Sci U S A, 2017. 114(48): p. E10281-E10290.

60. Lundqvist, H., et al., Permeability of the blood-brain barrier in the rat after local proton irradiation. Acta Radiol Oncol, 1982. 21(4): p. 267-71.

61. van Vulpen, M., et al., Changes in blood-brain barrier permeability induced by radiotherapy: implications for timing of chemotherapy? (Review). Oncol Rep, 2002. 9(4): p. 683-8.

62. Khatri, A., et al., Effect of radiation on the penetration of irinotecan in rat cerebrospinal fluid. Cancer Chemother Pharmacol, 2011. 68(3): p. 721-31.

63. Wilson, C.M., et al., Radiation-induced astrogliosis and blood-brain barrier damage can be abrogated using anti-TNF treatment. Int J Radiat Oncol Biol Phys, 2009. 74(3): p. 934-41.

64. FitzGerald, T.J., et al., The effect of radiation therapy on normal tissue function. Hematol Oncol Clin North Am, 2006. 20(1): p. 141-63.

65. Nordal, R.A. and C.S. Wong, Molecular targets in radiation-induced blood-brain barrier disruption. Int J Radiat Oncol Biol Phys, 2005. 62(1): p. 279-87. 
66. Deng, Z., et al., Distinct Expression of Various Angiogenesis Factors in Mice Brain After Whole-Brain Irradiation by X-ray. Neurochem Res, 2017. 42(2): p. 625-633. 67. Sandor, N., et al., Low dose cranial irradiation-induced cerebrovascular damage is reversible in mice. PLoS One, 2014. 9(11): p. e112397.

68. Pena, L.A., Z. Fuks, and R.N. Kolesnick, Radiation-induced apoptosis of endothelial cells in the murine central nervous system: protection by fibroblast growth factor and sphingomyelinase deficiency. Cancer Res, 2000. 60(2): p. 321-7.

69. Mildenberger, M., et al., An animal model of prophylactic cranial irradiation: histologic effects at acute, early and delayed stages. Int J Radiat Oncol Biol Phys, 1990. 18(5): p. 1051-60.

70. Kyrkanides, S., et al., TNF alpha and IL-1beta mediate intercellular adhesion molecule-1 induction via microglia-astrocyte interaction in CNS radiation injury. $\mathrm{J}$ Neuroimmunol, 1999. 95(1-2): p. 95-106.

71. Hong, J.H., et al., Induction of acute phase gene expression by brain irradiation. Int J Radiat Oncol Biol Phys, 1995. 33(3): p. 619-26.

72. Kyrkanides, S., et al., Cyclooxygenase-2 modulates brain inflammation-related gene expression in central nervous system radiation injury. Brain Res Mol Brain Res, 2002. 104(2): p. 159-69.

73. Yang, R., et al., Inhibitors of HIF-1alpha and CXCR4 Mitigate the Development of Radiation Necrosis in Mouse Brain. Int J Radiat Oncol Biol Phys, 2018. 100(4): p. 10161025.

74. Yuan, H., et al., Radiation-induced permeability and leukocyte adhesion in the rat blood-brain barrier: modulation with anti-ICAM-1 antibodies. Brain Res, 2003. 969(1-2): p. 59-69. 
75. Nakata, H., et al., Early blood-brain barrier disruption after high-dose singlefraction irradiation in rats. Acta Neurochir (Wien), 1995. 136(1-2): p. 82-6; discussion 867.

76. Crowe, W., et al., MRI Evaluation of the effects of Whole Brain Radiotherapy on Breast Cancer Brain Metastasis. Int J Radiat Biol, 2018: p. 1-27.

77. Bouchet, A., et al., Permeability of Brain Tumor Vessels Induced by Uniform or Spatially Microfractionated Synchrotron Radiation Therapies. Int J Radiat Oncol Biol Phys, 2017. 98(5): p. 1174-1182.

78. Merali, Z., et al., Evolution of blood-brain-barrier permeability after acute ischemic stroke. PLoS One, 2017. 12(2): p. e0171558.

79. Teng, F., et al., Blood-tumor barrier opening changes in brain metastases from pre to one-month post radiation therapy. Radiother Oncol, 2017. 125(1): p. 89-93.

80. Zeng, Y.D., et al., Blood-brain barrier permeability of gefitinib in patients with brain metastases from non-small-cell lung cancer before and during whole brain radiation therapy. Oncotarget, 2015. 6(10): p. 8366-76.

81. Lim, W.H., et al., Does radiation therapy increase gadolinium accumulation in the brain?: Quantitative analysis of T1 shortening using R1 relaxometry in glioblastoma multiforme patients. PLoS One, 2018. 13(2): p. e0192838.

82. Neuwelt, E.A., et al., Osmotic blood-brain barrier disruption. Computerized tomographic monitoring of chemotherapeutic agent delivery. J Clin Invest, 1979. 64(2): p. 684-8.

83. Rapoport, S.I., Osmotic opening of the blood-brain barrier: principles, mechanism, and therapeutic applications. Cell Mol Neurobiol, 2000. 20(2): p. 217-30. 84. Wilhelm, I., et al., Hyperosmotic stress induces Axl activation and cleavage in cerebral endothelial cells. J Neurochem, 2008. 107(1): p. 116-26. 
85. Godinho, B., et al., Transvascular Delivery of Hydrophobically Modified siRNAs: Gene Silencing in the Rat Brain upon Disruption of the Blood-Brain Barrier. Mol Ther, 2018. 26(11): p. 2580-2591. 


\section{Glossary}

Active Transport: The movement of molecules into the cell across the cellular membrane assisted by enzymes.

Blood-Brain Barrier: The physicochemical barrier existing at the interface between the systemic circulation (blood) and brain limiting the passive and active transport of small molecules, proteins, toxins, and other potentially pathogenic entities into the brain.

Blood-Brain Barrier Disruption: A physical opening, transient or persistent, of the BBB or BTB through a variety of mechanisms with the intent of increasing distribution of therapeutics into brain tissues.

Blood-Tumor Barrier: Similar to the BBB in healthy individuals, the BTB is the interface between the blood and metastatic or primary tumor cells. This barrier is inherently "leaky" due to lack of tight junctions and neo-angiogenesis induced by the tumor.

Brain Metastases: Tumors formed in the brain by cancer cells that have detached and migrated from a primary tumor site. 
Central Nervous System: Comprised of the brain and spinal cord, this complex of nerves controls the activities of the body.

Endothelial Cells: Cells that line the interior (luminal) surfaces of blood and lymphatic vessels.

Focused Transcranial Ultrasound: The use of low frequency ultrasonic waves, penetrating through the cranium to target particular sites within the brain.

Glioblastoma: Also known as glioblastoma multiforme. A form of primary CNS tumor arising from one of the glial cell types.

Neo-angiogenesis: The growth of new blood vessels.

Passive Diffusion: The movement of molecules across a membrane or between cells without the need for energy. Molecules down a concentration gradient, from a high concentration to a lower concentration.

Radiation Therapy: The use of X-rays, or similar forms of radiation, in the treatment of cancer. 


\begin{tabular}{|l|l|l|l|}
\hline \multicolumn{1}{|c|}{ Title } & Trial number & Mode of disruption & Type of disease \\
\hline Super-selective & NCT02861898 & Intra-arterial Mannitol & Glioblastoma \\
Repeated Infusion & & Brain Neoplasm, \\
of Cetuximab for & & Malignant EGFR \\
the Treatment of & & Gene \\
Newly Diagnosed & & Overexpression \\
Glioblastoma & & GBM \\
\hline Blood Brain Barrier & NCT03714243 & ExAblate Model 4000 & Her-2 positive \\
Disruption (BBBD) & & Type-2 & Breast Cancer, \\
Using MRgFUS in & & & Brain \\
the Treatment of & & & \\
Her2-positive & & & \\
Breast Cancer & & & \\
Brain Metastases & & & \\
(BBBD) & & & \\
\hline $\begin{array}{l}\text { ExAblate Blood } \\
\text { Brain Barrier }\end{array}$ & NCT03322813 & ExAblate 4000 - Type & GBM \\
for Planned & & & \\
\hline Gurgery in & & & \\
\hline Dlioblastoma & & & \\
\hline
\end{tabular}




\begin{tabular}{|l|l|l|l|}
\hline Assessment of & NCT03616860 & ExAblate Neuro Model & Glioblastoma \\
Safety and & 4000 Type 2.0 & \\
ExAblate Blood- & & \\
Brain Barrier (BBB) & & \\
Disruption for & & \\
Treatment of & & \\
Glioma & & & \\
\hline Blood-Brain Barrier & NCT02343991 & Transcranial ExABlate & Brain Tumor \\
Disruption Using & & & \\
Transcranial MRI- & & & \\
Guided Focused & & & \\
Ultrasound & & & \\
\hline ExAblate Blood- & NCT03712293 & ExAblate 4000 type 2.0 & Glioblastoma \\
Brain Barrier & & & \\
Disruption for & & & \\
Glioblastoma in & & & \\
Patients & & & \\
Undergoing & & & \\
\hline Chemotherapy & & & \\
\hline
\end{tabular}




\begin{tabular}{|c|c|c|c|}
\hline $\begin{array}{l}\text { Safety of BBB } \\
\text { Disruption Using } \\
\text { NaviFUS System in } \\
\text { Recurrent } \\
\text { Glioblastoma } \\
\text { Multiforme (GBM) } \\
\text { Patients }\end{array}$ & NCT03626896 & $\begin{array}{l}\text { Neuronavigation- } \\
\text { guided focus } \\
\text { ultrasound system } \\
\text { (NaviFUS) }\end{array}$ & $\begin{array}{l}\text { GBM, } \\
\text { Neoplasm, } \\
\text { glioma }\end{array}$ \\
\hline $\begin{array}{l}\text { Safety of BBB } \\
\text { Opening With the } \\
\text { SonoCloud } \\
\text { (SONOCLOUD) }\end{array}$ & NCT02253212 & SonoCloud & $\begin{array}{l}\text { Glioblastoma, } \\
\text { Glioma, Brain } \\
\text { Tumor }\end{array}$ \\
\hline $\begin{array}{l}\text { MRI Study of } \\
\text { Changes in Blood- } \\
\text { Brain/Tumor- } \\
\text { Barrier Permeability } \\
\text { in Patients With } \\
\text { Brain Metastases } \\
\text { During and After } \\
\text { Radiotherapy }\end{array}$ & NCT02031237 & $\begin{array}{l}\text { SRS, Fractinated } \\
\text { WBRT, Fractionated } \\
\text { SRS }\end{array}$ & $\begin{array}{l}\text { Brain } \\
\text { Metastases } \\
\text { (Breast, Lung, } \\
\text { Melanoma, etc.) }\end{array}$ \\
\hline
\end{tabular}

Table 2.1. BBB/BTB disruption techniques in ongoing or completed clinical trials. 


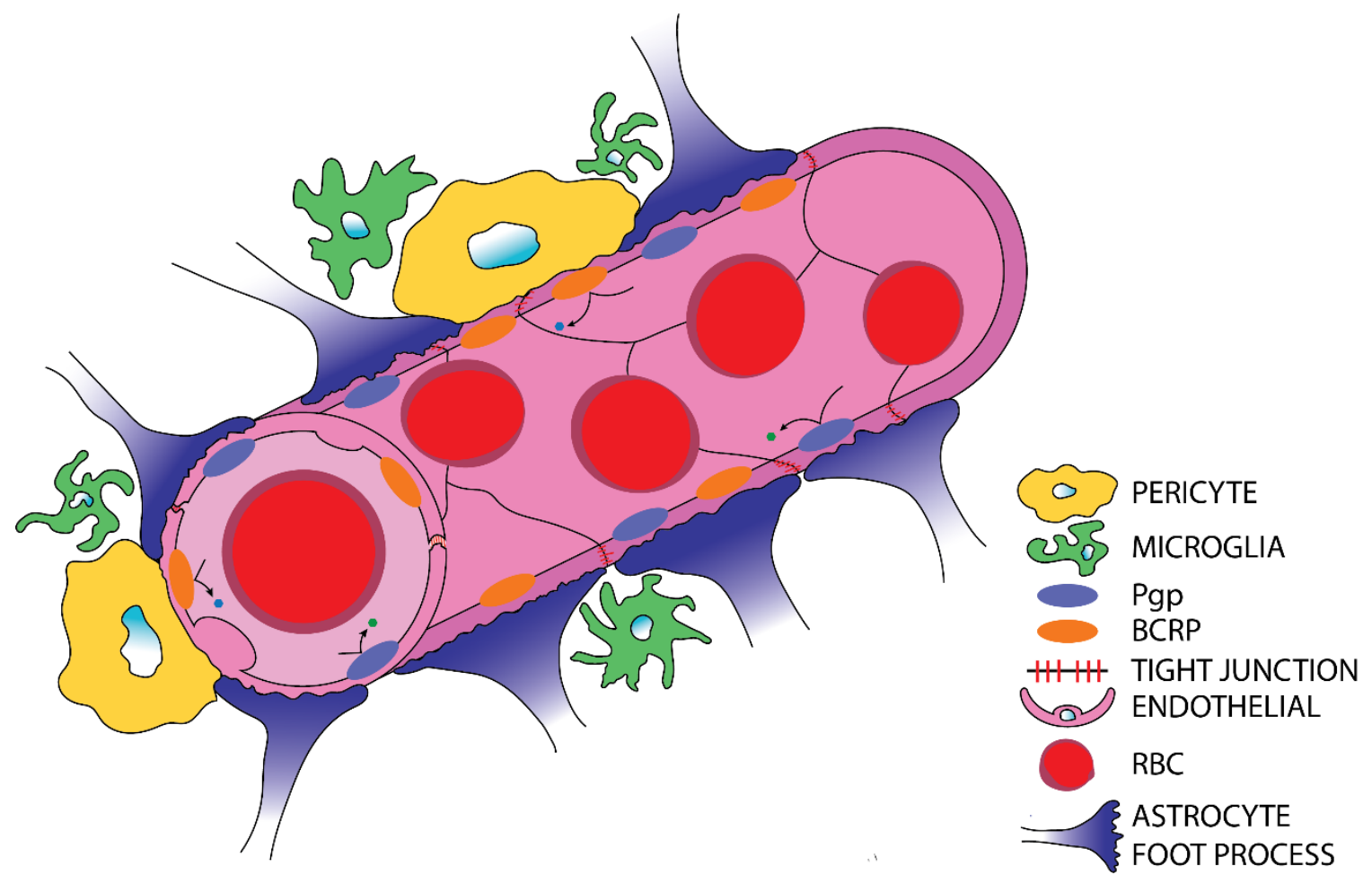

Figure 2.1. Normal blood-brain barrier anatomy and physiology. Brain capillary endothelial cells are tightly held to one another through continuous tight junction proteins and express P-glycoprotein (P-gp) and Breast cancer resistant protein (BCRP) efflux transporters. Astrocytic end-feet processes further seal and support BBB integrity. Pericytes further regulate cerebral blood flow and BBB permeability. Microglia, the brain's resident immune cells, can influence BBB permeability through inflammatory cascades and serve as the innate response to pathogens within the brain. 


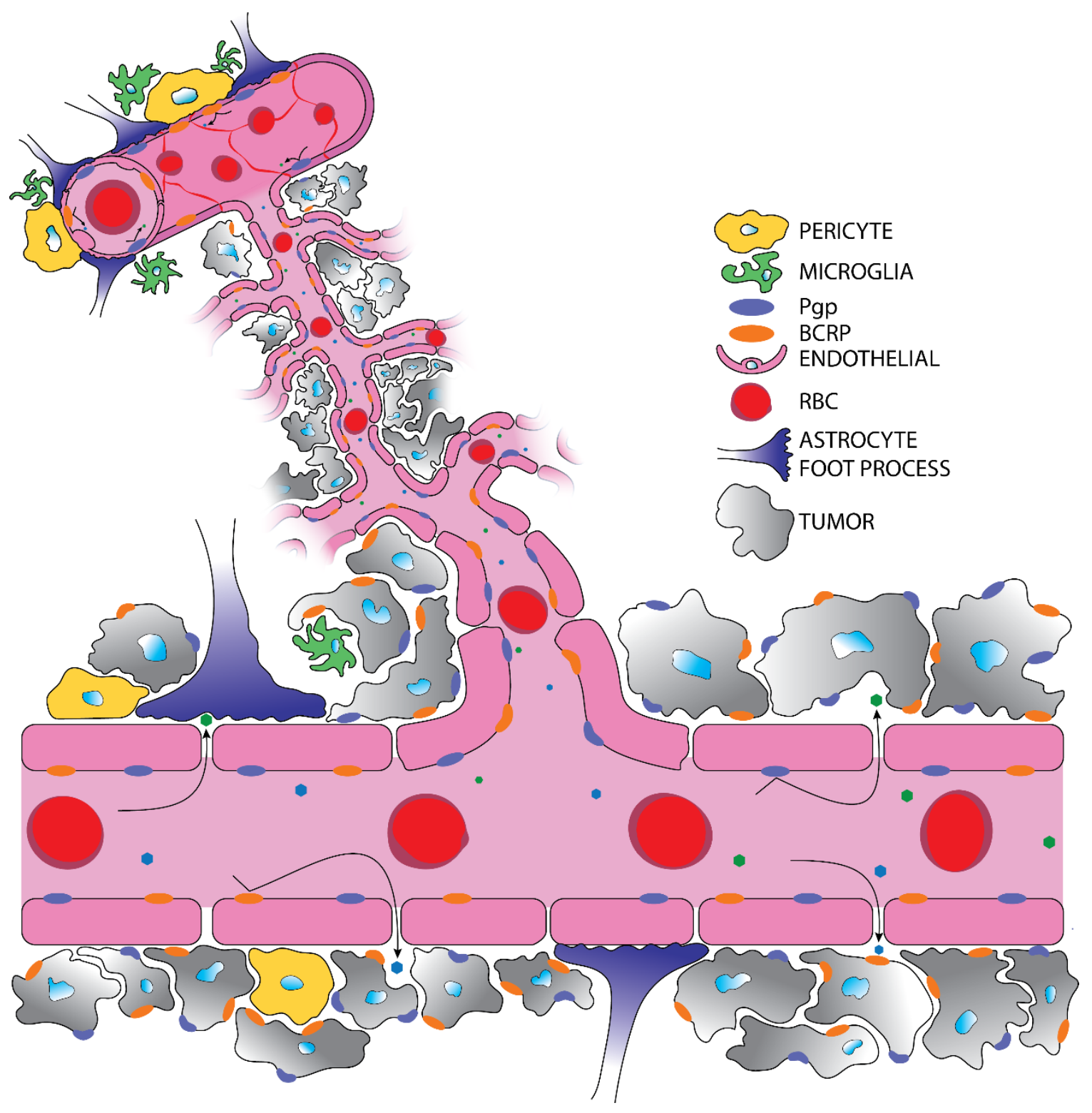

Figure 2.2. The blood-tumor barrier has altered anatomy and physiology.

Cancer cells coopt the cerebral vasculature and induce neo-angiogenesis resulting in fenestrated endothelia lacking tight junctional expression.

Fenestrated, mal-formed vasculature allows for heterogeneous uptake of drug solutes. Cancer cells have increased expression of the P-gp and BCRP efflux transporters. At the BTB, less astrocytic end-foot processes and pericytes exist contributing to altered BTB integrity. 


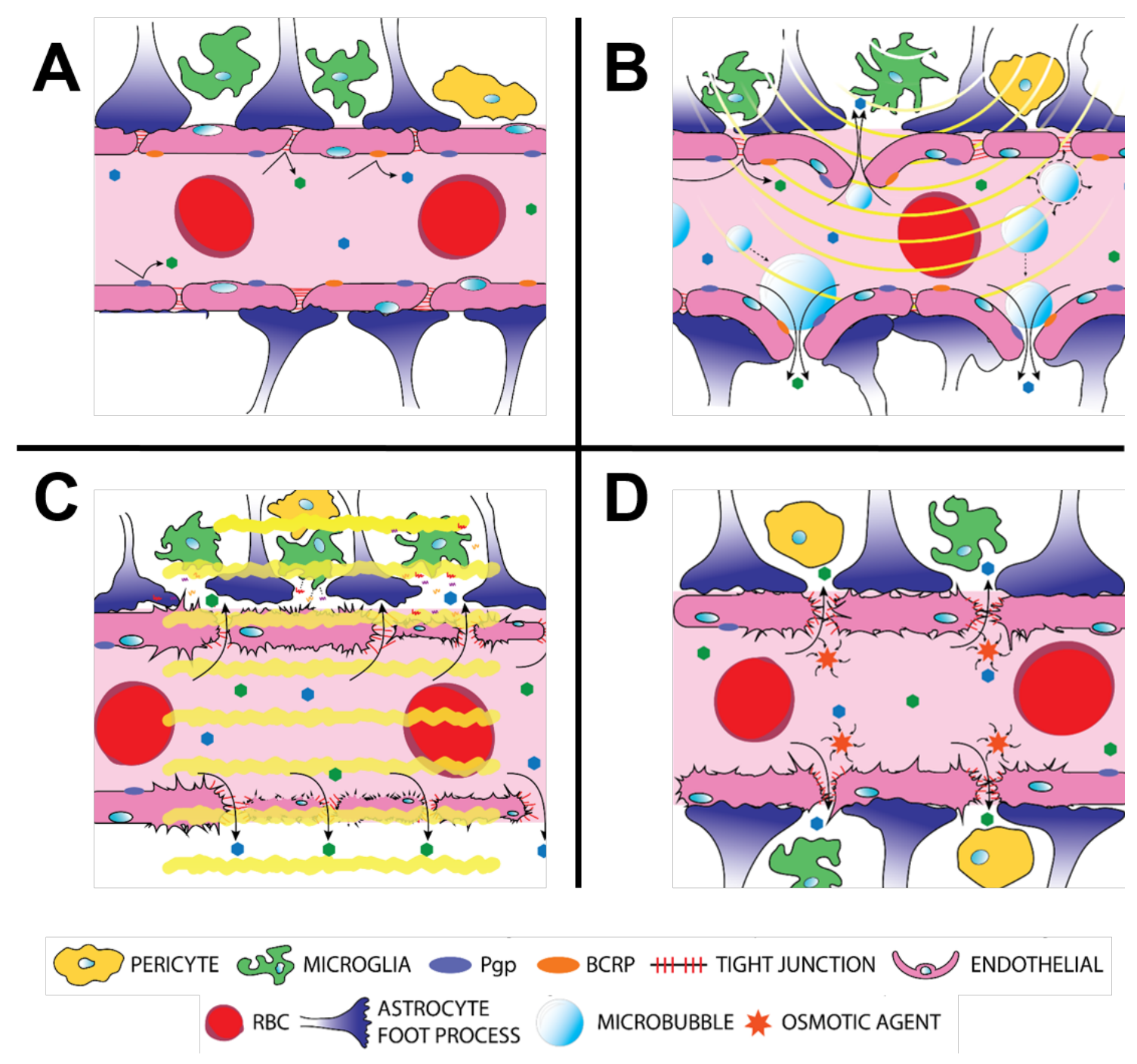

Figure 2.3. Blood-brain barrier disruption techniques. Normal, undisrupted BBB with non-fenestrated endothelial cells sealed by tight junction proteins, further supported by astrocytic end-feet, pericytes, and microglia (a). Focused ultrasound (yellow curves) in combination with intravenously injected disrupts the BBB through cavitation and acoustic forces, ultimately leading to decreased molecular expression of tight junction proteins and an inflammatory response (b). Radiation therapy (yellow lines) disrupts the BBB through mechanisms of endothelial cell death and a neuro-inflammatory response from astrocytes and 
microglial cells (c). Hyperosmotic solutions are able to induce contraction and shrinkage of endothelial cells through a calcium dependent mechanism prompting widening of tight junctions (d). 


\section{Chapter 3}

\section{The hypomethylating agent azacitidine is effective in treating brain metastasis of triple negative breast cancer through regulation of DNA methylation of keratin 18 gene.}

\subsection{Introduction}

Breast cancer is the second leading cause of cancer with the highest mortality rate in females in the United States (1). Based on genetic profiling, breast cancer is classified into four subtypes: luminal A (ER+ and/or PR+, HER2-, low Ki67 index), luminal B (ER+ and/or PR+, HER2+ or -, high Ki67 index), HER2 positive (HER2+, ER-, PR-), and triple negative (ER-, PR- and HER2-) (2). For drug treatment of receptor positive breast cancers, therapeutics that specifically target the hormone receptors and HER2 are available (3). However, for triple negative breast cancer, which is associated with an unfavorable prognosis, there are no targeted therapies, leaving patients with traditional chemotherapeutic agents that have significant adverse effects (4). In some patients, tumors metastasize to different locations within the body including lungs, liver, bones or brain. Once the lesion disseminates to brain, average patient survival time is less than one year, and treatments including chemotherapy, radiation and surgery are the primarily palliative options (5). 
DNA methylation is an epigenetic mechanism used by cells to control gene expression (6). In normal cells, DNA methylation ensures proper regulation of gene expression and silencing. Abnormal DNA hypermethylation, may result in dysregulation of these mechanisms resulting in altered gene function (7). Cancer is associated with altered DNA methylation leading to inhibition of tumor suppressor genes and compaction of chromatin $(8,9)$. Hypermethylation of numerous tumor suppressor genes is recognized in multiple cancer types and this phenomenon may contribute to the initiation and/or progression of the disease $(10,11)$. Of relevance to this study, multiple genes that are critical in breast carcinogenesis are hypermethylated including; the tumor suppressors p16, p53 and BRCA1, cell cycle regulator CCND2, and cell growth regulators ER and PR, as well as others (12-14).

Hypomethylating agents such as azacitidine (or azacytidine, AZA) and its deoxylderivative decitabine have been approved by the U.S. FDA to treat patients with hematological malignancies such myelodysplastic syndromes (MDS) (15). Although their use in breast cancer treatment are not approved for clinical use, DNA hypomethylating agents have been shown to activate tumor suppressor genes. It should be noted that when given intravenously, AZA crosses the bloodbrain barrier (BBB) and reaches cytotoxic levels in cerebrospinal fluid $(16,17)$. In this study, we evaluated the anti-tumor effects of the hypomethlyating agent AZA in a preclinical model of triple negative breast cancer brain metastasis and observed AZA has efficacy as a novel chemotherapeutic agent. 


\subsection{Materials and Methods}

Cell lines and chemicals

The parental regular triple negative breast cancer MDA-MB-231 (abbreviated as "231") cell line was purchased from ATCC (Manassas, VA). The brain colonizing counterpart of 231 cells (" $231 \mathrm{Br}$ " cells), also known as tropic or brain seeking 231 $\mathrm{Br}$ cells, were isolated from brain lesions in the brain metastasis of breast cancer mouse model we previously generated. These cells were kindly provided by $\mathrm{Dr}$. Patricia Steeg from the National Institute of Health Center for Cancer Research. Both cell lines were cultured at 37 oC, $5 \%$ CO2, in Dulbecco's modification of eagle's medium (DMEM) (Corning, Inc., Corning, NY) containing 10\% fetal bovine serum (VitaScientific, Inc., College Park, MD), 10 mM L-glutamine (Thermo Fisher Scientific, Waltham, MA), and 1X penicillin/streptomycin (Thermo Fisher Scientific, Waltham, MA). AZA was purchased from Sigma-Aldrich Inc. (St. Louis, MO).

\section{Western blotting}

The Western blotting assay was described previously (18). Briefly, cell lysates were prepared in RIPA buffer and protein samples were loaded on a SDSpolyacrylamide gel, separated by electrophoresis and subsequently transferred to a PVDF membrane. Membranes were blocked with $5 \%$ milk in 1X TBS containing $0.05 \%(\mathrm{v} / \mathrm{v})$ Tween-20 for 4 hours at room temperature and washed seven times with 1X TBS and 1X TBST alternatively. Membranes were then 
incubated with primary antibody overnight at $4{ }^{\circ} \mathrm{C}$, followed by incubation with secondary antibody at room temperature for 1 hour. Pierce supersignal

chemiluminescent substrates were used and images were captured by using the G:BOX Chemi XX9 gel doc system (Syngene Inc., Frederick, MD). Detailed information of the antibodies is listed in Supp. Table 1.

\section{MTT assay}

The cell viability was measured using the MTT assay kit (ATCC, Inc., Manassas, VA) and the manufactory's protocol was followed. Briefly, 1,000 cells in $100 \square \mathrm{I}$ were plated in each well in a 96-well plate and incubated overnight. On the next day, the cell medium was replenished and various concentrations of AZA were added to each well (triplicate) accordingly and incubated at $37^{\circ} \mathrm{C}$ for 72 hours. After incubation, $10 \square \mathrm{l}$ of MTT reagent was added to each well and the plate was incubate at $37^{\circ} \mathrm{C}$ for 4 hours. $1000 \square$ l of detergent reagent was then added to each well and the plate was left at room temperature in the dark for 4 hours. The optical density of absorbance at $570 \mathrm{~nm}$ was recorded using a Synergy 2 multimode microplate reader (Biotek, Inc., Winooski, VT). The cell viability was calculated based on the optical density value normalized to blank control. The IC50 of AZA in 231 and $231 \mathrm{Br}$ cells were calculated based on the cell viability measured by three independent MTT assays.

Apoptosis assay 
Cell apoptosis was measured using the PE Annexin V apoptosis detection kit (BD Biosciences, Inc., San Jose, CA). The manufacturer's protocol was followed and the percentage of apoptotic cells was detected and analyzed using the BD Accuri C6 flow cytometry (BD Biosciences, Inc., San Jose, CA).

\section{ELISA assay}

After cells were treated with various concentrations of AZA for 72 hours, the secreted VEGF in the medium was measured by using the human VEGF ELISA kit (Sigma-Aldrich, Inc., Saint Louis, MO). The ELISA assay was described previously, and the manufacture's protocol was followed (19). Briefly, $100 \square \mathrm{l}$ of each standard and medium sample was mixed and added into 96-well plates and incubated for 2.5 hours at room temperature with gentle shaking. The supernatant was then discarded and washed 4 times with $1 \mathrm{X}$ washing solution. $100 \square \mathrm{I}$ of 1X prepared biotinylated detection antibody was added for 1 hour at room temperature with gentle shaking. The solution was discarded and washed, and $100 \square \mathrm{I}$ of prepared HRP-conjugated streptavidin solution was added and incubated for 45 minutes at room temperature with gentle shaking. The solution was discarded and $100 \square \mathrm{I}$ of ELISA colorimetric TMB reagent was added and incubated for 10 minutes at room temperature in the dark with gentle shaking. Finally, $50 \square \mathrm{l}$ of stop solution was added and the plate was read at $450 \mathrm{~nm}$ using a Synergy2 multi-mode microplate reader (Biotek, Inc., Winooski, VT). The amount of VEGF present in the cell culture medium was normalized to the number of cells present at the time of collection. 
In vitro cell migration assay

The transwell migration assay was described previously $(20,21)$. After cells were treated with various concentration of AZA for 72 hours, they were washed with PBS and resuspended in serum-free medium. $600 \square \mathrm{I}$ of regular medium containing $10 \%$ serum was added to one well of a 24 -well plate, then the migration chamber (Millipore Inc, PI8P01250) was replaced in the well. $100 \square$ I of serum-free medium was first added in each chamber, then a total of 105 cells in $200 \square \mathrm{L}$ serum-free medium was added to the chamber. The plates were incubated at $37^{\circ} \mathrm{C}$ for various times $(3,16$, and 72 hours). At the end of the designated time point, medium in the chamber was removed and the chambers were gently washed twice with PBS. Cells were fixed with formaldehyde (3.7\% in PBS) at room temperature for 20 minutes, followed by PBS wash and permeabilization by $100 \%$ methanol at room temperature for 20 minutes. After removal of methanol and washing with PBS, cells were stained with $1 \%$ crystal violet at room temperature for 20 minutes. Excess crystal violet was removed and cells were washed with PBS. Finally, cells on the chamber were counted under the light microscope (average number of five microscope fields).

\section{In vitro cell invasion assay}

The cell invasion assay was described previously $(20,21)$. 24-well plates containing matrigel invasion chambers (Corning Inc., Corning, NY) were preincubated at $37^{\circ} \mathrm{C}$ overnight. Similar to the procedure used in the cell migration 
assay, the same number of cells (105 cells in $200 \square$ I serum-free medium) were plated in each well, and the plates were incubated at $37^{\circ} \mathrm{C}$ for pre-designated periods $(16,72$, and 96 hours). After reaching the time point, cells were fixed, permeabilized, stained, and counted under the light microscope using the same techniques as the cell migration assay.

\section{Wound-healing assay}

The wound-healing assay (also known as in vitro scratch assay) has been described previously $(20,21) .106$ of the 231 and $231 \mathrm{Br}$ cells were plated in sixwell plates and incubated at $37^{\circ} \mathrm{C}$ overnight. On the next day, after confirming that the cells were attached to the well and cell confluence reached $\sim 70 \%$, a scratch was made in each well using a $1 \mathrm{~mL}$ pipette tip, and medium containing increasing concentrations of AZA was added to each replicate. The number of cells present in the scratch made on day 0 was counted for each pre-designated time $(24,48,72$, and 96 hours $)$ and pictures of the denuded area were taken using an Olympus IX50 inverted system microscope (Olympus, Inc., Center Valley, PA) every day for 5 days.

\section{Detection of the keratin 18 gene by PCR}

DNA from both cell lines was extracted and purified using the GeneJet genomic DNA purification kit (Thermo Fisher Scientific, Waltham, MA) based on the manufacturer's protocol. The pair of primers designed to measure the keratin 18 gene by PCR is forward 5'-CTGGCCTCTTACCTGGACAGAGTGAG-3'and 
reverse 5'-TGT GGCTAGGTGCGCGGATGGAAATCC-3', which yields a 300bp PCR product. The PCR reaction was set up by using the iProof high-fidelity PCR kit (Bio-Rad Laboratories, Inc., Hercules, CA) and was performed with an Eppendorf Mastercycler thermocycler (Hamburg, Germany). The PCR thermal cycling protocol was as follow: initial denaturation at $98^{\circ} \mathrm{C}$ for 30 seconds, denaturation at $98^{\circ} \mathrm{C}$ for 10 seconds, annealing at $65^{\circ} \mathrm{C}$ for 30 seconds, and extension at $72{ }^{\circ} \mathrm{C}$ for 30 seconds, a total of 30 cycles, and final extension at 72 ${ }^{\circ} \mathrm{C}$ for 10 minutes.

Real-time PCR

The real-time PCR procedure was described previously (18). Briefly, cells were harvested by centrifugation at $1500 \mathrm{~g}$ for 5 minutes at $4^{\circ} \mathrm{C}$, resuspended in $250 \mu \mathrm{l}$ 1X PBS, then lysed by adding $750 \mu \mathrm{l}$ Trizol LS reagent (Invitrogen, Inc., Carlsbad, CA). RNA was then isolated following the manufacturer's protocol and was subsequently resuspended in $30 \mu \mathrm{L}$ of RNase-free water. The RNA concentration was measured using a Synergy 2 multi-mode microplate reader (BioTek Inc., Winooski, VT). The TURBO DNA-free kit (Thermo Fisher Scientific Inc., Waltham, MA) was used to remove DNA contamination within each sample. The first-strand cDNA was synthesized using the SuperScript III first-strand synthesis system (Thermo Fisher Scientific Inc., Waltham, MA) following the manufacturer's protocol. FAM-MGB primer/probe mixes for keratin 18 (Hs02827483_g1), VEGFA (Hs00900055_m1), and GAPDH (Hs02786624_ were used for real-time PCR TaqMan gene expression assays (Applied 
Biosystems Inc., Foster City, CA). All real-time PCR reactions were performed in duplicate with no-RT control and water control on the StepOnePlus real time PCR system (Applied Biosystems Inc., Foster City, CA). Individual cDNA samples were normalized according to their levels of GAPDH and the relative standard curve method was used for analysis.

Sequencing of the intron 1 region of the keratin 18 gene in both cell lines In order to compare the DNA sequence of intron 1 region $(737 \mathrm{bp})$ of the keratin 18 gene between 231 and $231 \mathrm{Br}$ cells, we designed a pair of primers and used PCR to amplify the desired region. The forward sequence was 5'GATCATCGAGGACCTGAGGG-3', the reverse sequence was 5'-GGGGAGC AGATCCTTCTTAGC-3'. The PCR reaction was set up using the DreamTaq hot start green DNA polymerase kit (ThermoFisher Scientific, Inc., Waltham, MA) and PCR was performed with the Bio-Rad MJ mini personal thermal cycler \# PTC114 (Bio-Rad Laboratories, Inc., Hercules, CA). The PCR thermal cycling protocol was as follow: initial denaturation at $95{ }^{\circ} \mathrm{C}$ for 2 minutes, denaturation at $95^{\circ} \mathrm{C}$ for 30 seconds, annealing at $60^{\circ} \mathrm{C}$ for 30 seconds, and extension at $72{ }^{\circ} \mathrm{C}$ for 45 seconds, a total of 19 cycles, then followed by dropping $0.5^{\circ} \mathrm{C}$ each time to $50^{\circ} \mathrm{C}, 95^{\circ} \mathrm{C}$ for 30 seconds, $50{ }^{\circ} \mathrm{C}$ for 30 seconds, $72{ }^{\circ} \mathrm{C}$ for 30 seconds, a total of 19 times. The final extension was at $72{ }^{\circ} \mathrm{C}$ for 10 minutes. This yielded a single and clear 906bp PCR product. The PCR product was cloned into the pCR2.1-TOPO vector using the TOPO TA cloning kit (Invitrogen, Inc., Carlsbad, CA) following the manufacturer's protocol. The cloning product was then 
transformed into DH5-alpha E.coli competent cells (Invitrogen, Inc., Carlsbad, CA). The transformations were spread on ampicillin-selective plates and incubated overnight at $37^{\circ} \mathrm{C}$. Colonies were picked and cultured in LB medium containing $100 \square \mathrm{g} / \mathrm{ml}$ ampicillin with shaking at $250 \mathrm{RPM}$ overnight at $37^{\circ} \mathrm{C}$. On the next day, plasmid DNA was isolated by using the Invitrogen PureLink quick plasmid miniprep kit (Invitrogen, Inc., Carlsbad, CA). Plasmid DNA samples from five positive colonies were send to the West Virginia University Genomics Core Facility for sequencing.

Keratin 18 gene DNA methylation determination by bisulfite chemical modification

The genomic DNA from 231 and $231 \mathrm{Br}$ cells was isolated using the GeneJET genomic DNA purification kit (ThermoFisher Scientific, Inc., Waltham, MA) following the manufacturer's protocol. The genomic DNA was then treated with bisulfite to chemically modify non-methylated cytosines into uracil using the EZ DNA methylation-lightning kit (Zymo Research, Inc., Irvine, CA) following the manufacurer's protocol. In this treatment, unmethylated cytosine residues were converted to uracil, while methylated cytosine residues were resistant to bisulfite modification and remained as a cytosine residue. In order to measure and compare the DNA methylation of the keratin 18 gene in both cell lines, five pairs of primers were designed and used to fully cover and amplify the bisulfite modified intron 1 region of keratin 18 gene by PCR. The sequences of the five pairs of primers were: Pair 1 forward: 5'- TTAATTATYGGTTTTTG 
GGTTTTGTTTAGG-3', reverse: 5'-RATCTCCAAACTCCTCA CTCTAT-3'; Pair 2 forward: 5'-TTGGATAGAGTGAGGAGTTTGGAGA-3', reverse: 5'AAAAATCCAAATATACCC AACCCCCT-3'; Pair 3 forward: 5'GGAGGGGGTTGGGTATATTT-3', reverse: 5'-CACCC TAAATTAACTCCTCCCAAAA-3'; Pair 4 forward: 5'TTGAGTTATTTAGGAGTAAAT AAGAGGTTTTTTTTTG-3', reverse: 5'CCAAAAATAACCAAAAACTCTCCCTAAA-3'; Pair 5 forward: 5'TGGTTATTTTTGGGATTAGGAAGTTTTTATTAG-3', reverse: 5'-CAAA ATCCCACTATAAACCCCTAACT-3'. The methods used in the PCR reaction set up and performance, TOPO TA cloning, and plasmid DNA isolation were the same as described above. Each of the five pairs of primers yielded a single and clear PCR band using bisulfite converted genomic DNA as the template from both cell lines (Supp. Fig. 4). Finally, plasmid DNA samples from five positive colonies generated from each pair of primers were sent to West Virginia University Genomics Core Facility for sequencing.

Hhal restriction digestion

The Hhal restriction enzyme was purchased from New England BioLabs, Inc. (Ipswich, MA). $20 \square$ restriction digestion reaction containing $500 \mathrm{ng}$ genomic DNA isolated from 231 or $231 \mathrm{Br}$ cells and $1 \square \mathrm{l} \mathrm{Hhal} \mathrm{was} \mathrm{set} \mathrm{up} \mathrm{following} \mathrm{the}$ manufacturer's protocol. The reaction was incubated in a $37^{\circ} \mathrm{C}$ water bath overnight allowing the full digestion of DNA by Hhal. The pair of primers designed used to detect the Hhal digestion site is forward: 5'- 
GGAGGGGGTTGGG CATACT-3', reverse: 5'-

CACCCTGGATTGGCTCCTCCCAAAG-3'. If DNA methylation prevented

digestion of the keratin 18 gene, the DNA would not be digested by Hhal and this primer would yield a $\sim 300 \mathrm{bp} \mathrm{PCR}$ product. On the contrary, if the DNA was digested by Hhal, no such a $\sim 300 \mathrm{bp}$ PCR product would be formed. A pair of primers designed and used as positive control to detect the keratin 18 gene was forward: 5'-AGCTAGAC AAGTACTGGTCTCAGCAG-3', reverse 5'CAGCTCTGACTCAAGGTGCAGCAGGAT-3'. Regardless of digestion status of the keratin 18 gene digested by Hhal, this primer could detect the presence of the keratin 18 yielding a $\sim 300 \mathrm{bp}$ PCR product in both cell lines. Methods used in PCR reaction set up and performance were the same as described above.

Survival of animals with preclinical brain metastases of breast cancer treated with $A Z A$

Animal experiments were approved by the Institutional Animal Care and Use Committee at the West Virginia University. Use of the brain metastasis breast cancer in vivo mouse model has been described previously $(22,23)$. Briefly, the brain tropic breast cancer 231Br cells were isolated by repeated cycles of intracardiac injection of the parental 231 cells, harvesting of brain metastases, and ex vivo culture of isolated cells. These brain metastatic cells were injected into the left cardiac ventricle, circulated in the peripheral vasculature, arrested in brain capillaries, with subsequent extravasation across the in vivo BBB, and developed metastatic lesions in mice. The presence of metastatic tumors was 
confirmed on day 21 after intracardiac injection with bioluminescent imaging (BLI) using the IVIS Spectrum CT imaging system (PerkinElmer, Waltham, MA). After tumor burden confirmation on day 21 , mice were intraperitoneally administered with AZA $(2.5 \mathrm{mg} / \mathrm{kg})$ or vehicle control (PBS) in a total of four cycles. In each cycle, AZA or PBS was injected for five continuous days, stopped for two days, and then again followed by another five continuous days. Between each cycle, there was a two-week off interval. Tumor burden was monitored twice weekly and quantified using BLI assay, similar to our previous work $(22,23)$. Mice were introperitoneally injected with D-luciferin potassium salt $(150 \mathrm{mg} / \mathrm{kg}$ body weight, PerkinElmer Inc., Waltham, MA) and then the brain bioluminescent signal was captured 15 minutes after injection of luciferin. Animals were euthanized under anesthesia at the presentation of neurological symptoms or when moribund. Statistics. Statistical significance of the data between two groups was analyzed by the Student t-test (Prism 8). Statistical significance of the data with more than two groups was analyzed by one-way ANOVA with a Tukey post-test (Prism 8). Significance levels were set at $p<0.05\left(^{*}\right), p<0.01\left(^{* *}\right)$, and $\left.p<0.001{ }^{(* *}\right)$.

\subsection{Results}

Brain colonizing breast cancer cells display a different growth pattern compared to parental breast cancer cells.

After intracardiac injection, the parental triple negative breast cancer cells (231) are disseminated throughout the body (Fig. 3.1a), while the brain colonizing cells 
$(231 \mathrm{Br})$ are primarily distributed to the brain (Fig. 3.1b).. The fold change of cell growth suggest the $231 \mathrm{Br}$ cells replicate faster than the 231 cells (Fig. 3.1c).

Brain colonizing breast cancer cells are more sensitive to AZA treatment compared to regular cancer cells.

The IC50 value of AZA in 231Br and 231 cells was determined using an MTT assay. We observed IC50 values for AZA was $83.3 \pm 8.8 \square \mathrm{M}$ in $231 \mathrm{Br}$ cells and $48 \pm 4.9 \square \mathrm{M}$ in 231 cells ( $p<0.01$, Fig. 3.2a and Supp. Fig. 3.1a), suggesting differential sensitivity to AZA. In subsequent experiments both lines were treated with a range of AZA concentrations $(0-500 \square \mathrm{M})$ for 72 hours, and apoptosis was calculated by a percentage of Annexin-V positive cells using flow cytometry. At concentrations of $20 \square \mathrm{M}$ and $100 \square \mathrm{M}$ of AZA, a greater degree of apoptosis was induced in $231 \mathrm{Br}$ cells compared to 231 cells $(p<0.001)$. However, $500 \mathrm{uM}$ of AZA caused apoptosis at similar level in both cell types (Fig. 3.2b and Supp. Fig. 3.1b). The expression of the anti-apoptotic protein BCL-2 decreased in $231 \mathrm{Br}$ cells upon AZA treatment, with little effect in the 231 cells. However, expression BCL-XL was not detected in either cell lines (Fig. 3.2c and Supp. Table 3.2). Further, there was an increased expression of the pro-apoptotic proteins caspase- 3 and caspase-9, when AZA was exposed to the $231 \mathrm{Br}$ cells in a dosedependent manner (Fig. 3.2d and Supp. Table 3.2). However, expression of two other two pro-apoptotic proteins BAD and BAX proteins remained unchanged after AZA treatment in (Supp. Fig. 3.1c). 
AZA inhibited the Wnt signaling transduction pathway in brain colonizing breast cancer cells.

Treatment with AZA in $231 \mathrm{Br}$ cells for 72 hours inhibited expression of Wnt-3, Wnt-4, glycogen synthase kinase-3 (GSK-3), and beta-catenin in a dose dependent manner as determined by a western blot (Fig. 3.3a). In contrast, treatment with AZA in 231 cells did not change Wnt-3 expression significantly, but at a higher concentration (100 $\square \mathrm{M})$, AZA inhibited expression of Wnt-4, GSK3 and beta-catenin in these cells (Fig. 3.3a). AZA treatment had no effect on Wnt-1 expression in either cell types and the expression of Wnt-5, Wnt-11, and adenomatous polyposis coli (APC) was undetectable in both lines (Supp. Fig. 3.2a). While there were changes in the Wnt signaling transduction pathway there was no significant impact on either the Ras/Raf/MEK/MAPK or the PI3K/Akt/mTOR pathways in either cell line (Supp. Fig. 3.2b and 3.2c).

Angiogenesis related markers were decreased by with AZA treatment in both cell lines.

Angiogenesis-related markers were measured in both cell lines treated by different concentrations of AZA. At higher concentrations (100 $\square \mathrm{M})$, AZA treatment for 72 hours decreased expression of vascular endothelial growth factor (VEGF) receptor 2 only in the 231Br cells. Treatment with AZA had no significant impact on hypoxia-inducible factor (HIF)-1 alpha expression in either cell lines (Fig. 3.3b). However, expression of VEGF, VEGF-receptor 1, and transforming growth factor (TGF) beta was undetectable in either types of cells 
(Data not shown). The mRNA was measured by real-time PCR (Fig. 3.3c) and the secreted VEGF into the cell culture medium was measured by ELISA assay (Fig.3d) in both lines treated with AZA. Cellular VEGF mRNA level in 231 cells was significantly higher in 231 cells compared to $231 \mathrm{Br}$ cells $(p<0.001)$ and AZA treatment did not have significant impact on VEGF mRNA levels in both cell lines (Fig. 3.3c). However, AZA reduced the amount of VEGF secreted into the medium in a dose-dependent manner in both cell lines (Fig. 3.3d).

Brain colonizing cells have higher migration and invasion potential. After incubation, we did not observe significant changes in cell number with AZA at early time points, but after 72 hours of incubation, $231 \mathrm{Br}$ cells had higher migration potential ( $p<0.001$, Fig. $4 a$ and Supp. Fig. 3.3a). Similarly, in the cell invasion assay, at early time points (16 and 72 hours) there were no significant differences between the cell lines, (Supp. Fig. 3.3b) but at 96 hours $231 \mathrm{Br}$ cells (without AZA) had increased migration ( $p<0.05$, Fig. 3.4b). Of interest, AZA treatment did not significantly impact cell invasion in either lines (Fig. 3.4b and Supp. Fig. 3.3b). Consistent with cell migration and invasion assays, the wound healing assay showed that the $231 \mathrm{Br}$ cells migrated faster than 231 cells. After 72 hours of AZA treatment, the width of wound was still present in 231 cells, but not in $231 \mathrm{Br}$ cells (Supp. Fig. 3.3d-3.3e). In addition, AZA treatment significantly inhibited wound healing in both lines after 48 hours (Fig. 3.4c and Supp. Fig. 3.3c-3.3e). 
Hallmark metastasis markers are higher in the brain colonizing cell line. Matrix metalloproteinases (MMPs) such as MMP2, MMP7 and MMP9 are metastasis markers in breast cancer $(24,25)$. MMP2 was expressed in both cell lines but greater in 231Br cells, and MMP9 expression was not seen in 231 cells but detected in $231 \mathrm{Br}$ cells. Interestingly, AZA treatment for 72 hours had no significant impact on expression of MMP2 and MMP9 in either cell line (Fig. 3.4d). Epithelial markers including E-cadherin and cytokeratins, and mesenchymal markers $\mathrm{N}$-cadherin were measured with and without $\mathrm{AZA}$ treatment in both cell lines (26). Expression of E-cadherin was not detected in either cell line (Data not shown), while vimentin was present at similar levels in both cell types. $\mathrm{N}$-cadherin expression was only detected in $231 \mathrm{Br}$ cells. Treatment with AZA decreased expression of N-cadherin in 231 cells (Fig. 3.4d). Since cytokeratin expression is decreased during the EMT process (27), we measured their expression profile using a pan-cytokeratin antibody mixture of $\mathrm{AE} 1$ and $\mathrm{AE} 3$. This was done to detect multiple members of the cytokeratin family of proteins (1-6, 7-10, 14-16, and 19). We observed expression of cytokeratins measured by the pan-cytokeratin antibody was lower in $231 \mathrm{Br}$ cells compared to 231 cells. In addition, higher concentrations of AZA (50 $\square \mathrm{M}$ and 100 M) treatment further decreased expression in the 231Br line (Fig. 3.4d).

DNA methylation is altered in brain colonizing cells compared to parental cancer cells. 
Cytokeratin 18 is an epithelial cytokeratin coded by the keratin 18 (or KRT18) gene (28). We detected expression of keratin 18 protein in 231 cells but not in $231 \mathrm{Br}$ cells, despite the presence of the keratin-18 gene being present as detected by real-time PCR (Fig. 3.5a and 3.5b). Also, it should be noted that the mRNA levels of the keratin gene 18 were significantly lower in the $231 \mathrm{Br}$ cells $(p<0.001$, Fig. $3.5 c)$. We noted that AZA treatment increased keratin 18KRT18 mRNA significantly in a dose dependent manner in 231Br cells (every dose increased with significance at $p<0.05$ or lower, Fig. 3.5c). Based on this data, we hypothesized that decreased gene expression may be due to DNA hypermethylation. It has been shown DNA methylation of intron 1 in the keratin 18 gene is important in regulating expression (29). We did not detect DNA mutation or deletion of this region (737 bp) of the keratin 18 gene in ether cell line, and the DNA sequence is identical (Data not shown). We sequenced and compared DNA methylation of the intron 1 region in the two cell lines by using the bisulfite conversion method. We observed three cytosine residues were converted into uracil in 231 cells while they remained unchanged in $231 \mathrm{Br}$ cells (Fig. 3.5d). Among the three identified different cytosine sites, one is the target of the Hhal restriction enzyme (Fig. 3.5d). To further confirm the difference in particular cytosine methylation, the genomic DNA from both cell lines was digested with Hhal following PCR using designed primers. A 300bp band was detected in $231 \mathrm{Br}$ cells, but not in 231 cells, confirming cytosine methylation of keratin gene (Fig. 3.5e). DNA methylation is catalyzed by DNA methyltransferases (DNMTs) which include two major types DNMT3a and 
DNMT3b and accordingly we measured the expression of the enzymes with AZA treatment (6). The expression of DNMT3a was only detected in $231 \mathrm{Br}$ cells and the expression of DNMT3b was undetectable in either cell types (Fig. 3.5f). AZA treatment decreases the DNMT3a expression in 231Br cells indicating AZA can potentially inhibit the process of DNA methylation in brain colonizing cells (Fig. $3.5 f)$.

AZA decreased tumor burden and prolonged survival in mice with brain metastases of breast cancer.

To determine if AZA treatment improved survival and control of tumor burden in vivo, we injected mice intracardially with the $231 \mathrm{Br}$ cells and allowed for development of metastatic brain lesions $(22,23)$. After 21 days, tumor bearing mice were randomized into vehicle (PBS) and drug treatment (AZA, $2.5 \mathrm{mg} / \mathrm{kg}$ body weight) groups. We observed that in $2.5 \mathrm{mg} / \mathrm{kg}$ AZA treated mice, tumor burden was significantly lower compared to vehicle treated mice $(p=0.0112$, Fig. 3.6a). We also noted that AZA treatment significantly increased survival when compared to vehicle treated mice, with the median survival of 50 and 42 days respectively ( $p=0.0026$, Fig. 3.6b).

\subsection{Discussion}

DNA methylation is an epigenetic mechanism used by cells to control gene expression (6). DNA hypermethylation may cause improper gene silencing, leading to the downregulation of gene expression and alleviation of gene 
function. Hypermethylation of numerous tumor suppressor genes has been identified in multiple cancer types suggesting that DNA hypermethylation may contribute to the initiation, development, and increased metastatic capacity of cancer $(7,10)$.

The hypomethylating agent AZA and its deoxyl derivative 2'-deoxy-5'-azacytidine (decitabine) were developed as pyrimidine nucleoside analogs in 1960s. Later, it was observed that the compounds inhibit DNA methylation in human cell lines (30). Studies of AZA also showed antitumor activity in hematological malignancies including MDS, acute myeloid, chronic myeloid, and acute lymphocytic leukemia (31). Concurrent epigenetic work showed that multiple important genes are hypermethylated in MDS patients. One of the genes is tumor suppressor gene CDKN2B which encodes the cyclin dependent kinase inhibitor p15INK4b. Other hypermethlated genes in MDS patients include the calcitonin gene, HIC, E-cadherin and estrogen receptor (32). The led to the FDA approval of AZA as the first therapy for all subtypes of MDS in 2004 (33). With relevance to this work, in breast cancer multiple genes (e.g., p16, p53, and BRAC1) are also hypermethylated (12-14). Breast cancer brain metastasis poses a lifethreatening problem for women with advanced metastatic breast cancer and current chemotherapeutic agents are largely ineffective against brain metastases (34-38). In this study, we tested the effectiveness of the hypomethylating agent AZA in treating brain metastasis of breast cancer using a combined in vitro cell and in vivo approach $(22,23)$. 
We observed that the IC50 value of AZA in $231 \mathrm{Br}$ cells was significantly lower than in 231Br cells (Fig. 3.2a) and AZA treatment triggered a higher percentage of apoptosis in $231 \mathrm{Br}$ cells compared to 231 cells (Figs. 3.2b-3.2d). Further, AZA inhibited BCL-2 expression in 231Br cells in a dose-dependent manner suggesting inhibition of anti-apoptotic BCL-2 may be a mechanism of anti-tumor therapeutic response induced by AZA in these cells. Overall, these results suggest $231 \mathrm{Br}$ cells are more sensitive to AZA treatment. In triple negative breast cancer, Wnt signaling regulates cell differentiation, proliferation and stem cell pluripotency $(39,40)$. Accordingly, we examined AZA effects in Wnt signaling, and observed expression of Wnt-3, Wnt-4, GSK-3, and beta-catenin were inhibited by AZA in a dose dependent manner in $231 \mathrm{Br}$ cells (Fig. 3.3a). As betacatenin is required for the tumorigenic behavior of triple-negative cancer cells, our results suggest AZA inhibits Wnt signaling as well as tumorigenesis in brain colonizing cells more (41). Consistent with previous literature, we did not observe differences in the Ras/Raf/MEK/MAPK and PI3K/Akt/mTOR pathways as they have greater influence in HER2+ cancer (Supp. Fig. 3.2b and 3.2c) (42). We also observed that AZA treatment inhibits of angiogenesis related markers (Fig. 3.3) and cell migration and invasion (Fig. 3.3a). Collectively, in vitro results support the hypothesis that AZA is effective in treating brain metastasis of breast cancer in vivo. 
The BBB acts as a physiological and biochemical barrier that restricts the passage of many hydrophilic and large molecular weight compounds. AZA is a nucleic acid synthesis inhibitor with a molecular weight of $244.2 \mathrm{~g} / \mathrm{mole}$ and a XLogP3 of -1.9 . Strictly based on its physicochemical properties, AZA serves as a model compound to cross biological membranes like the BBB with an ideal range of molecular weight, lipophilicity, and hydrogen bond donors and acceptors. Additionally, its relatively high aqueous solubility and stability renders it as a potentially advantageous investigative chemotherapeutic for brain delivery (43-46). Since AZA is able to cross the $\operatorname{BBB}(16,17)$, we next used an in vivo mouse model of brain metastasis of breast cancer and treated animals with AZA (2.5mg/kg body weight) or vehicle control (PBS). Our in vivo studies showed that the overall survival of AZA treated mice was significantly increased compared to mice treated with PBS (Fig. 3.6a). The in vivo BLI assay also suggested that AZA significantly inhibited the tumor activity in mice compared to PBS (Fig. 3.6b).

Decitabine has been shown to reverse gefitinib resistance caused by DAPK gene promoter methylation in lung cancer cells, suggesting a role of DNA methylation in drug resistance and cancer progression (47). Thus, after confirming the effectiveness of AZA in treating brain metastasis triple negative breast cancer in vitro and in vivo, we explored the molecular mechanism of action of AZA. EMT is defined by the loss of epithelial and acquisition of mesenchymal characteristics, which promotes cancer cell progression, invasion, and metastasis into surrounding microenvironment $(48,49)$. Cytokeratins are major structural 
proteins found in epithelial cells, forming the cytoplasmic network of intermediate filaments (50). As important epithelial makers, the expression of cytokeratins is decreased during the EMT process, which may contribute to breast cancer metastasis (27). The human cytokeratin family consists of at least 20 members coded by different cytokeratin genes including the keratin 18 gene, which is located on chromosome $12 q 13$ with 3791 bp $(28,51)$. Keratin 18 plays biological functions in carcinogenesis and its expression may serve as a differential diagnostic marker in various cancers such as small cell lung cancer and breast cancer (52-54). In order to explore role of keratin 18 gene in breast cancer brain metastasis and hypomethylating agent treatment, we first measured the expression of the keratin $18 \mathrm{DNA}, \mathrm{mRNA}$ and protein. We observed the keratin 18 gene was present in both cell lines (Fig. 3.5b), but its transcription and translation were dramatically decreased in $231 \mathrm{Br}$ cells (Fig. 3.5c and 3.5a). Moreover, AZA treatment increased the mRNA level of keratin 18 in a dose dependent manner (Fig. 3.5c). Previous studies have shown that the first intron of the keratin 18 gene contains GC rich regions with DNA methylation sites, which are important in regulating its expression $(29,55)$. Thus, we hypothesized that the decreased keratin 18 gene expression in $231 \mathrm{Br}$ cells is due to DNA hypermethylation. We sequenced and compared the DNA sequence of intron 1 (737 bp) of the keratin 18 gene between both cell lines and we found the DNA sequence was identical, further indicating decreased expression of keratin 18 in $231 \mathrm{Br}$ cells may due to DNA hypermethylation. Further, we identified three cytosines that were converted into uracil in 231 cells, suggesting DNA 
methylation status of keratin 18 was different between both cell lines (Fig. 3.5d). Further Hhal restriction enzyme digestion following PCR analysis confirmed at least one of the three cytosine sites is methylated in $231 \mathrm{Br}$ but not in 231 cells (Fig. 3.5e). These results strongly suggest that the keratin 18 gene is hypermethylated in $231 \mathrm{Br}$ cells.

So far, two mechanisms of action of hypomethylating agents have been reported: (i) incorporation of drugs into DNA strands and prevention of RNA synthesis; (ii) inhibition of the activity of DNMTs which catalyze the process of DNA methylation $(6,7,11)$. Targeting DNA methylation may be the general mechanism of these agents, however, the precise mechanism of action of hypomethylating agents in cancer treatment has not been elucidated. DNMTs are enzymes that catalyze the addition of methyl groups to cytosine residues in DNA. DNMTs found in mammalian cells include DNMT1, DNMT3a, DNMT3b. DNMT1 and DNMT3b are found to plays roles in the development of central nervous system while DNMT3a has important functions in acute myeloid leukemia (56-59). We measured the expression of DNMT3a and DNMT3b and found the expression of DNMT3a is only present in $231 \mathrm{Br}$ cells while DNMT3b expression is undetectable in either cell lines. We also noticed that AZA inhibited the DNMT3a expression in $231 \mathrm{Br}$ cells (Fig. 3.5f). The presence of DNMT3a and inhibition of its expression by AZA in the brain colonizing cells suggests (1) DNA methylation is elevated and (2) AZA inhibits DNA methylation in these cells. Comparing different methylation status of the keratin 18 gene between the two cell lines, and the difference in 
DNMT3a expression provides a correlation and explanation of DNA methylation, brain metastasis, and effectiveness of AZA in brain colonizing cells.

\subsection{Conclusion}

In summary, based upon our findings, we believe the DNA hypomethylating agent AZA may represent as a new class of chemotherapeutic agents and a novel therapy for treatment of brain metastasis of breast cancer. A recent study showed that epigenetically reprogrammed genomic methylation serves as a universal cancer biomarker (58). We propose DNA hypermethylation of the keratin 18 gene may serve as a biomarker for diagnosis of brain metastasis of breast cancer, or can be used to evaluate whether breast cancer patients with brain metastasis are potential candidates and that would benefit from hypomethylating agent treatment. Moreover, the hypermethylated keratin 18 gene may be a potential drug target that can be used for the development of novel targeted therapy drugs in treating patients with brain metastasis breast cancer. 


\subsection{References}

1. Siegel RL, Miller KD, Jemal A. Cancer statistics, 2018. CA: a cancer journal for clinicians 68, 7-30 (2018).

2. Yersal O, Barutca S. Biological subtypes of breast cancer: Prognostic and therapeutic implications. World journal of clinical oncology 5, 412-424 (2014).

3. Waks AG, Winer EP. Breast Cancer Treatment: A Review. Jama 321, 288-300 (2019).

4. Anampa J, Makower D, Sparano JA. Progress in adjuvant chemotherapy for breast cancer: an overview. BMC medicine 13, 195 (2015).

5. Leone JP, Leone BA. Breast cancer brain metastases: the last frontier. Experimental hematology \& oncology 4, 33 (2015).

6. Moore LD, Le T, Fan G. DNA methylation and its basic function. Neuropsychopharmacology : official publication of the American College of Neuropsychopharmacology 38, 23-38 (2013).

7. Baylin SB. DNA methylation and gene silencing in cancer. Nature clinical practice Oncology 2 Suppl 1, S4-11 (2005).

8. Baylin SB, Ohm JE. Epigenetic gene silencing in cancer - a mechanism for early oncogenic pathway addiction? Nature reviews Cancer 6, 107-116 (2006).

9. Esteller M. Cancer epigenomics: DNA methylomes and histonemodification maps. Nature reviews Genetics 8, 286-298 (2007). 
10. Xia L, et al. CHD4 Has Oncogenic Functions in Initiating and Maintaining Epigenetic Suppression of Multiple Tumor Suppressor Genes. Cancer cell 31, 653-668.e657 (2017).

11. Santos FP, Kantarjian H, Garcia-Manero G, Issa JP, Ravandi F. Decitabine in the treatment of myelodysplastic syndromes. Expert review of anticancer therapy 10, 9-22 (2010).

12. Widschwendter $M$, Jones PA. DNA methylation and breast carcinogenesis. Oncogene 21, 5462-5482 (2002).

13. Szyf M. DNA methylation signatures for breast cancer classification and prognosis. Genome medicine 4, 26 (2012).

14. Wu Y, Sarkissyan M, Vadgama JV. Epigenetics in breast and prostate cancer. Methods in molecular biology (Clifton, NJ) 1238, 425-466 (2015).

15. Welch JS, Petti AA, Ley TJ. Decitabine in TP53-Mutated AML. The New England journal of medicine 376, 797-798 (2017).

16. Syed N, et al. Epigenetic status of argininosuccinate synthetase and argininosuccinate lyase modulates autophagy and cell death in glioblastoma. Cell death \& disease 4, e458 (2013).

17. Chabot GG, Rivard GE, Momparler RL. Plasma and cerebrospinal fluid pharmacokinetics of 5-Aza-2'-deoxycytidine in rabbits and dogs. Cancer research 43, 592-597 (1983).

18. Liu T, et al. Knockdown of HSPA9 induces TP53-dependent apoptosis in human hematopoietic progenitor cells. PloS one 12, e0170470 (2017). 
19. Coppe JP, Kauser K, Campisi J, Beausejour CM. Secretion of vascular endothelial growth factor by primary human fibroblasts at senescence. The Journal of biological chemistry 281, 29568-29574 (2006).

20. Turner DP, Moussa O, Sauane M, Fisher PB, Watson DK. Prostatederived ETS factor is a mediator of metastatic potential through the inhibition of migration and invasion in breast cancer. Cancer research 67, 1618-1625 (2007). 21. Chen Y, Lu B, Yang Q, Fearns C, Yates JR, 3rd, Lee JD. Combined integrin phosphoproteomic analyses and small interfering RNA--based functional screening identify key regulators for cancer cell adhesion and migration. Cancer research 69, 3713-3720 (2009).

22. Adkins $\mathrm{CE}$, et al. Characterization of passive permeability at the bloodtumor barrier in five preclinical models of brain metastases of breast cancer. Clinical \& experimental metastasis 33, 373-383 (2016).

23. Adkins CE, et al. A novel preclinical method to quantitatively evaluate early-stage metastatic events at the murine blood-brain barrier. Cancer prevention research (Philadelphia, Pa) 8, 68-76 (2015).

24. Weigelt B, Peterse JL, van 't Veer LJ. Breast cancer metastasis: markers and models. Nature reviews Cancer 5, 591-602 (2005).

25. Merdad A, et al. Expression of matrix metalloproteinases (MMPs) in primary human breast cancer: MMP-9 as a potential biomarker for cancer invasion and metastasis. Anticancer research 34, 1355-1366 (2014). 
26. Gout S, Huot J. Role of cancer microenvironment in metastasis: focus on colon cancer. Cancer microenvironment : official journal of the International Cancer Microenvironment Society 1, 69-83 (2008).

27. Zeisberg M, Neilson EG. Biomarkers for epithelial-mesenchymal transitions. The Journal of clinical investigation 119, 1429-1437 (2009).

28. Schweizer J, et al. New consensus nomenclature for mammalian keratins. The Journal of cell biology 174, 169-174 (2006).

29. Umezawa A, Yamamoto H, Rhodes K, Klemsz MJ, Maki RA, Oshima RG. Methylation of an ETS site in the intron enhancer of the keratin 18 gene participates in tissue-specific repression. Molecular and cellular biology 17, 48854894 (1997).

30. Stresemann C, Lyko F. Modes of action of the DNA methyltransferase inhibitors azacytidine and decitabine. International journal of cancer 123, 8-13 (2008).

31. Fratta E, Montico B, Rizzo A, Colizzi F, Sigalotti L, Dolcetti R. Epimutational profile of hematologic malignancies as attractive target for new epigenetic therapies. Oncotarget 7, 57327-57350 (2016).

32. Raj K, Mufti GJ. Azacytidine (Vidaza(R)) in the treatment of myelodysplastic syndromes. Therapeutics and clinical risk management 2, 377388 (2006).

33. Müller A, Florek M. 5-Azacytidine/Azacitidine. Small Molecules in Oncology 184, 159-170 (2010). 
34. Bent vd. The role of chemotherapy in brain metastases. Eur J Cancer 39, 2114-2120 (2003).

35. Ekenel M, Hormigo A, Peak S, DeAngelis L, Abrey L. Capecitabine therapy of central nervous system metastases from breast cancer. J Neurooncol 85, 223-227 (2007).

36. Rivera E, et al. Phase I study of capecitabine in combination with temozolomide in the treatment of patients with brain metastases of breast cancer. Cancer 107, 1348-1354 (2006).

37. Lin N, et al. Phase II trial of lapatinib for brain metastases in patients with HER2+ breast cancer. J Clin Oncol 24, 503 (2006).

38. Lin N, et al. Phase II trial of lapatinib for brain metasetases in patients with human epidermal growth factor receptor 2-positive breast cancer. Clin Cancer Res 26, 1993-1999 (2008).

39. De P, Carlson JH, Wu H, Marcus A, Leyland-Jones B, Dey N. Wnt-betacatenin pathway signals metastasis-associated tumor cell phenotypes in triple negative breast cancers. Oncotarget 7, 43124-43149 (2016).

40. Pohl SG, Brook N, Agostino M, Arfuso F, Kumar AP, Dharmarajan A. Wnt signaling in triple-negative breast cancer. Oncogenesis 6, e310 (2017).

41. Xu J, Prosperi JR, Choudhury N, Olopade OI, Goss KH. beta-Catenin is required for the tumorigenic behavior of triple-negative breast cancer cells. PloS one 10, e0117097 (2015). 
42. Fink MY, Chipuk JE. Survival of HER2-Positive Breast Cancer Cells: Receptor Signaling to Apoptotic Control Centers. Genes \& cancer 4, 187-195 (2013).

43. Benet LZ, Broccatelli F, Oprea TI. BDDCS applied to over 900 drugs. The AAPS journal 13, 519-547 (2011).

44. Dave RA, Morris ME. Novel high/low solubility classification methods for new molecular entities. International journal of pharmaceutics 511, 111-126 (2016).

45. McCormack SE, Warlick ED. Epigenetic approaches in the treatment of myelodysplastic syndromes: clinical utility of azacitidine. OncoTargets and therapy 3, 157-165 (2010).

46. Derissen EJ, Beijnen JH, Schellens JH. Concise drug review: azacitidine and decitabine. The oncologist 18, 619-624 (2013).

47. Yang B, Yang ZG, Gao B, Shao GG, Li GH. Epithelial-mesenchymal transition and breast cancer: role, molecular mechanisms and clinical impact. Int J Clin Exp Pathol. 8, 12961-6 (2015).

48. Foroni C, Broggini M, Generali D, Damia G. Epithelial-mesenchymal transition and breast cancer: role, molecular mechanisms and clinical impact. Cancer treatment reviews 38, 689-697 (2012).

49. Iwatsuki M, et al. Epithelial-mesenchymal transition in cancer development and its clinical significance. Cancer science 101, 293-299 (2010). 
50. Herrmann H, Bar H, Kreplak L, Strelkov SV, Aebi U. Intermediate filaments: from cell architecture to nanomechanics. Nature reviews Molecular cell biology 8, 562-573 (2007).

51. Weng YR, Cui Y, Fang JY. Biological functions of cytokeratin 18 in cancer. Molecular cancer research : MCR 10, 485-493 (2012).

52. Nagashio R, et al. Significant high expression of cytokeratins 7, 8, 18, 19 in pulmonary large cell neuroendocrine carcinomas, compared to small cell lung carcinomas. Pathology international 60, 71-77 (2010).

53. lyer SV, et al. Understanding the role of keratins 8 and 18 in neoplastic potential of breast cancer derived cell lines. PloS one 8, e53532 (2013).

54. Kulesh DA, Oshima RG. Cloning of the human keratin 18 gene and its expression in nonepithelial mouse cells. Molecular and cellular biology 8, 15401550 (1988).

55. Fan G, et al. DNA methylation controls the timing of astrogliogenesis through regulation of JAK-STAT signaling. Development (Cambridge, England) $132,3345-3356(2005)$.

56. Hutnick LK, et al. DNA hypomethylation restricted to the murine forebrain induces cortical degeneration and impairs postnatal neuronal maturation. Human molecular genetics 18, 2875-2888 (2009).

57. Cole CB, et al. PML-RARA requires DNA methyltransferase $3 A$ to initiate acute promyelocytic leukemia. The Journal of clinical investigation 126, 85-98 (2016). 
58. Ley TJ, et al. DNMT3A mutations in acute myeloid leukemia. The New England journal of medicine 363, 2424-2433 (2010).

59. Sina AA, et al. Epigenetically reprogrammed methylation landscape drives the DNA self-assembly and serves as a universal cancer biomarker. Nature communications 9, 4915 (2018). 


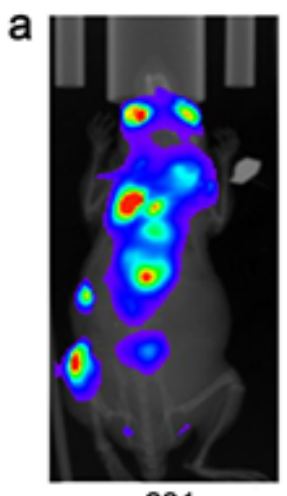

231

b

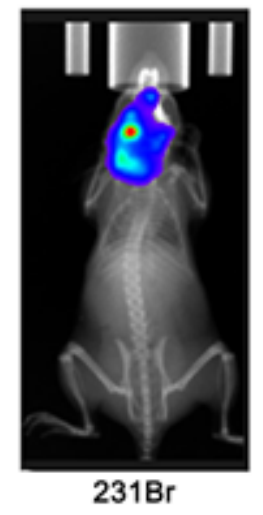

C

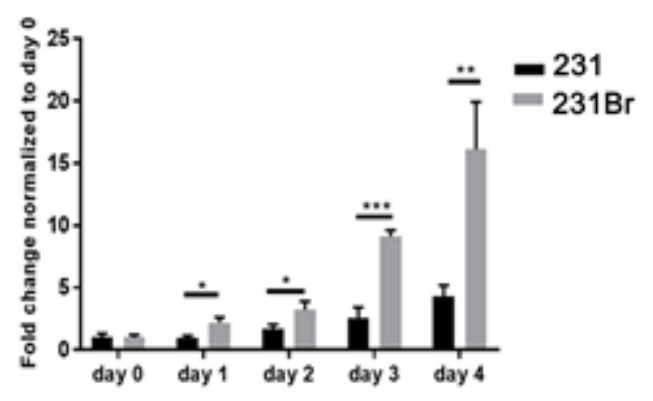

Fig. 3.1. Brain colonizing breast cancer cells $(231 \mathrm{Br})$ have a different oncological phenotype compared to parental breast cancer 231 cells. (a) After intracardiac injection, the parental triple negative breast cancer cells (231) are seen disseminated throughout the body of mice. (b) The brain colonizing triple negative breast cancer cells $(231 \mathrm{Br})$ primarily reside in the brain of mice. (c) 
$231 \mathrm{Br}$ cells grow significantly faster compared to 231 cells in vitro. Fold change of cell numbers in each day was compared between the two cell lines using Student's t-test. All error bars represent standard deviation (SD), N = 3 technical replicates, representative of two independent experiments. ${ }^{*} p<0.05,{ }^{* *} p<0.01$, ${ }^{* * *} p<0.001$.
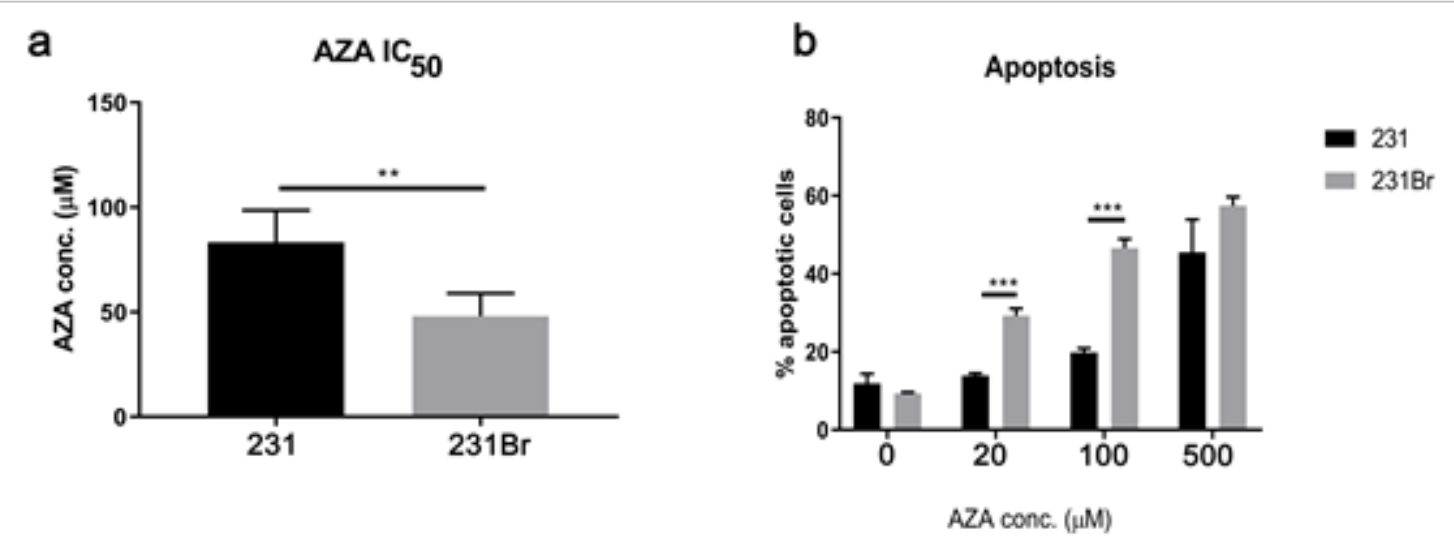

C

d
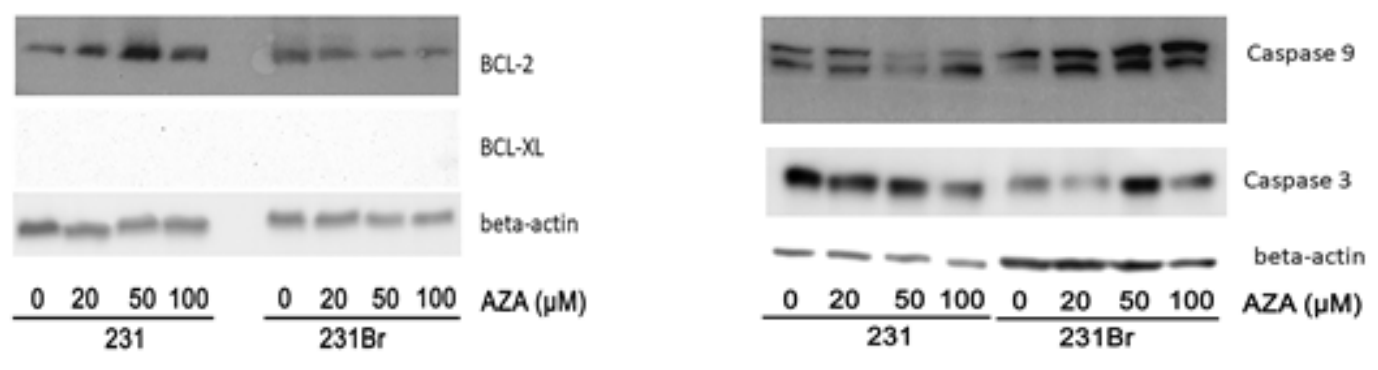

Fig. 3.2. 231Br breast cancer cells are more sensitive to AZA treatment compared to 231 cells. (a) IC50 values of AZA in both cell lines were calculated using the MTT assay. The IC50 value of AZA is $48 \pm 4.90 \square \mathrm{M}$ in $231 \mathrm{Br}$ cells and $83.33 \pm 8.82 \square \mathrm{M}$ in 231 cells ( $p<0.01$ ). (b) 231 and $231 \mathrm{Br}$ cells were treated with various concentrations of $A Z A$ for 72 hours and the Annexin- $V$ positive cells were 
considered as apoptotic cells. $20 \square \mathrm{M}$ and $100 \square \mathrm{M}$ of AZA treatment triggers higher percentage of apoptotic cells in $231 \mathrm{Br}$ cells compared to 231 cells. IC50 values and percentage of Annexin- $V$ positive cells were compared between the two cell lines using Student's t-test. All error bars represent standard deviation (SD), $\mathrm{N}=3$ technical replicates, representative of three independent experiments. ${ }^{*} p<0.05,{ }^{* *} p<0.01,{ }^{* * *} p<0.001$. (c) Expression of $B C L-2$ and $B C L-x L$ in 231 and $231 \mathrm{Br}$ cells after AZA treatment for 72 hours measured by Western blotting assay. Beta-actin was used as the loading control. The blots shown are a presentation of two independent experiments. (d) Expression of caspase-3 and caspase-9 in 231 and $231 \mathrm{Br}$ cells after AZA treatment for 72 hours. Beta-actin was used as the loading control. The blots shown are a presentation of two independent experiments. 
a

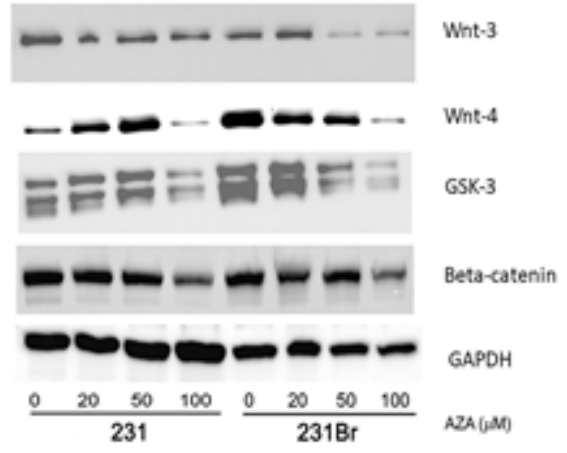

C

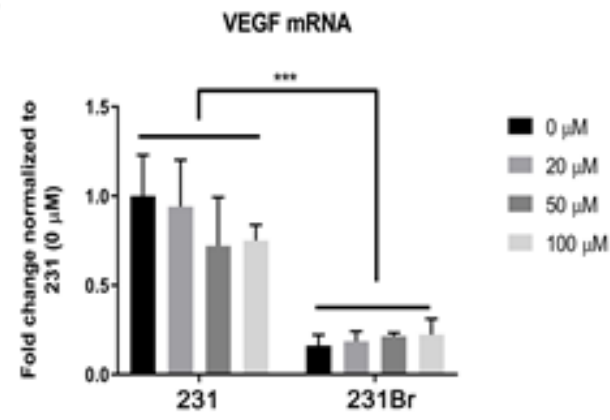

b

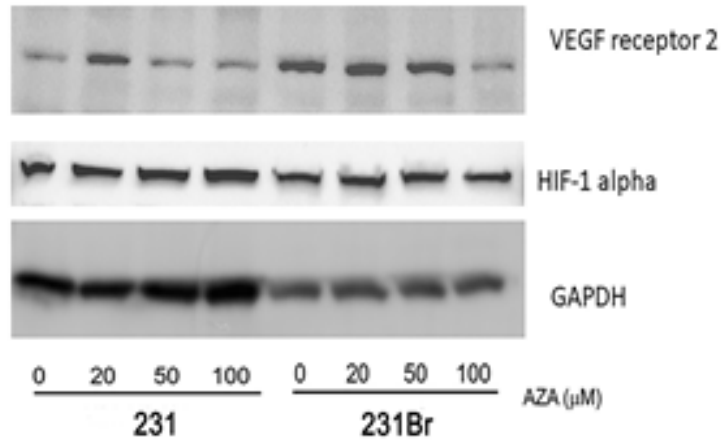

d

VEGF ELISA

Fig. 3.3. AZA differentially inhibits Wnt signaling transduction pathway and angiogenesis related markers in vitro. (a) Expression of Wnt-3, Wnt-4, GSK-4, and beta-catenin in 231 and $231 \mathrm{Br}$ cells after AZA treatment for 72 hours measured by Western blotting assay. GAPDH was used as the loading control. The blots shown are a presentation of two independent experiments. (b) Expression of VEGF receptor 2 and HIF-1 alpha in 231 and 231Br cells after AZA treatment for 72 hours measured by Western blotting assay. GAPDH was used as the loading control. The blots shown are a presentation of two independent experiments. (c). VEGF mRNA level in 231 and $231 \mathrm{Br}$ cells after AZA treatment for 72 hours measured by real-time PCR. All error bars represent standard 
deviation (SD), $\mathrm{N}=3$ technical replicates, representative of two independent experiments. ${ }^{*} p<0.05,{ }^{* *} p<0.01,{ }^{* * *} p<0.001$. (d) The amount of VEGF released into the cell culture medium of 231 and $231 \mathrm{Br}$ cells after 72 hours of AZA treatment measured by ELSIA. All error bars represent standard deviation (SD), $\mathrm{N}=3$ technical replicates, representative of two independent experiments. ${ }^{*} p<0.05,{ }^{* *} p<0.01,{ }^{* * *} p<0.001$
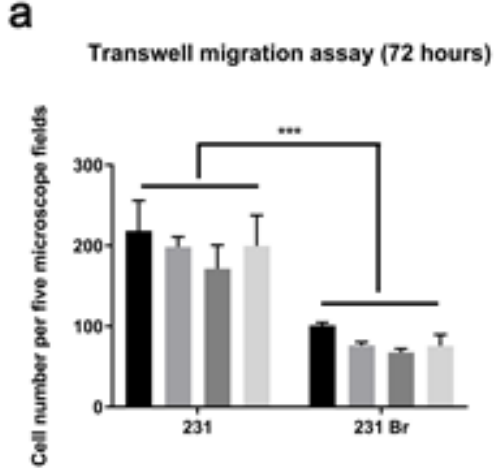

C

Wound-healing assay (72 hours)

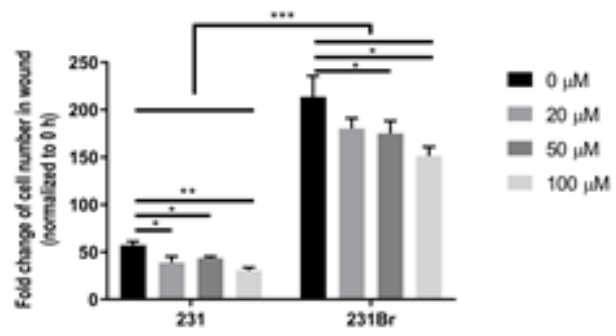

b Transwell invasion assay (96 hours)

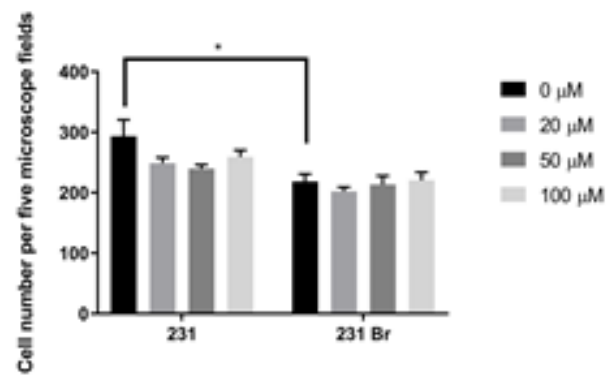

d

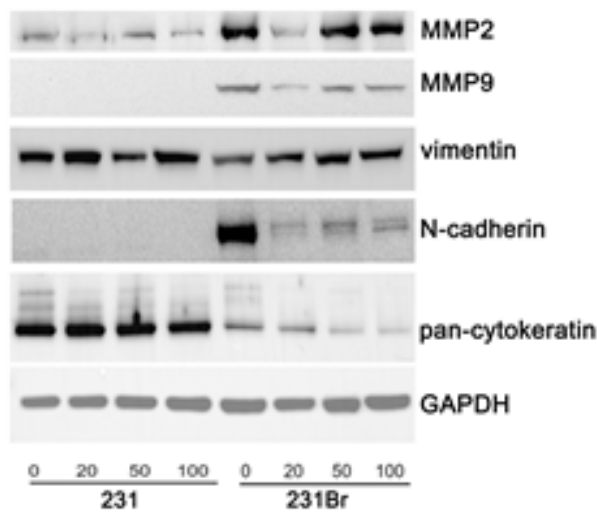

Fig. 3.4. 231Br cells have higher migration and invasion potential compared to 231 cells. (a) Quantification of cells migrating across transwells 72 hours after plating cells in the migration chambers measured by transwell migration 
assay. Y-axis stands for the average number of cell migration per 5 microscope fields. All error bars represent standard deviation (SD), representative of two independent experiments. ${ }^{*} p<0.05,{ }^{* *} p<0.01,{ }^{* * *} p<0.001$. (b) Quantification of cells migrating across transwells 96 hours after plating cells in the Matrigel coated migration chambers measured by transwell invasion assay. Y-axis stands for the average number of cell migration per 5 microscope fields. All error bars represent standard deviation (SD), representative of two independent experiments. ${ }^{*} p<0.05,{ }^{* *} p<0.01,{ }^{* * *} p<0.001$. (c) Quantification of cells presented in the scratch made on day 0 ( 0 hour time point) at 72 hours after AZA treatment by wound-healing assay. Cell numbers in the scratch wound were normalized to 0 hour. All error bars represent standard deviation (SD), representative of two independent experiments. ${ }^{*} p<0.05,{ }^{* *} p<0.01,{ }^{* * *} p<0.001$. (d) Expression of MMP2, MMP9, vimentin, $\mathrm{N}$-cadherin, and pan-cytokeratin in 231 and $231 \mathrm{Br}$ cells after AZA treatment for 72 hours measured by Western blotting assay. GAPDH was used as the loading control. The blots shown are a presentation of two independent experiments. 
a

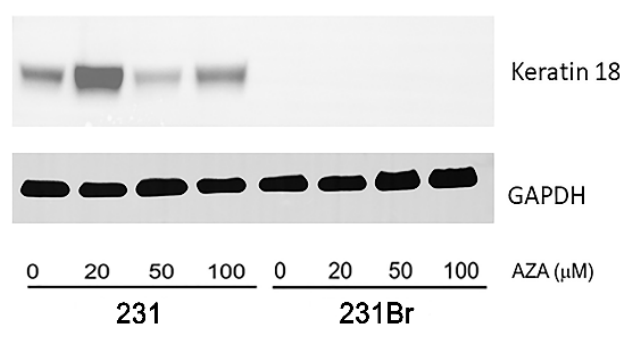

C

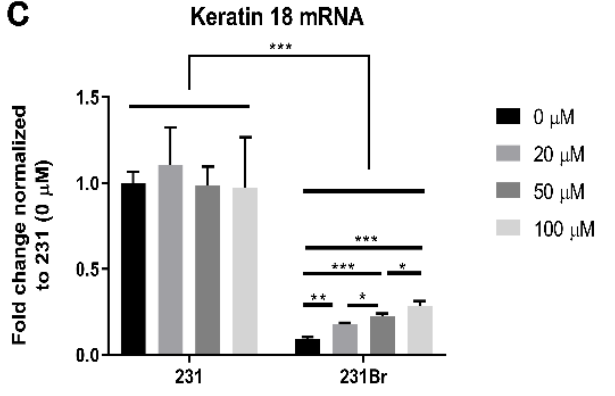

e

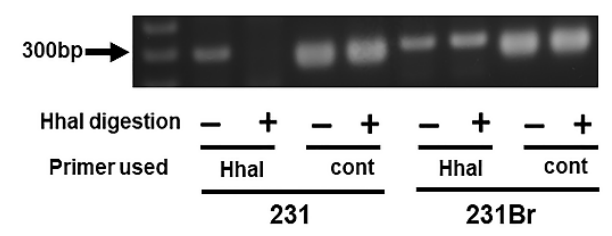

f
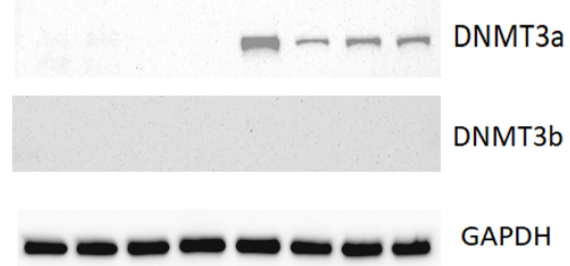

$\begin{array}{lllllll}0 \quad 20 \quad 50 \quad 100 & 0 \quad 20 \quad 50 \quad 100 & \text { AZA (uM) } \\ 231 & 231 \mathrm{Br} & \end{array}$ b

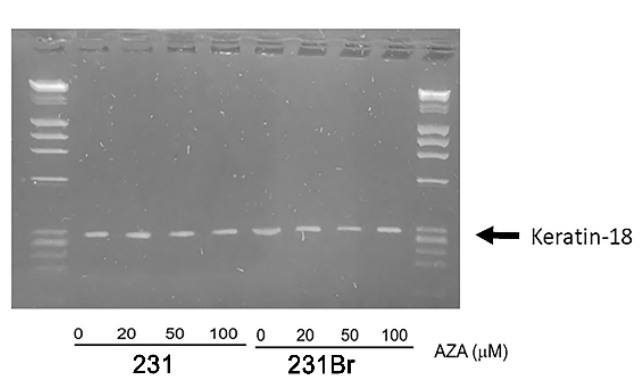

d

g9gagcach.t ggagaagaagggacceage tcagagachggagccattac LLeaagatca tcgaggacet gagggetcacgtaaggggta ggagggacctcaactcccag cettgtctga cectccaatt atacactccttgectcttt ccgtcattccataaccaccc aacccetact HhaI

ccaccgggag ggggttgggeatacctggat ttccatcc $\overline{g c g c a c c t a g c c}$ acagggtcco

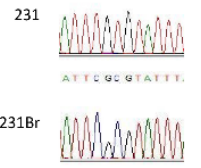

taagagcage agctaggcatgggagggetc tttcccaggagagaggggga aggggacagg gttgagagct ttacagaggaagtggacage atggagggaggtaaggaaag gectgtaaag aggaggagac actggctctggcggaatggg gactattggagggttaagcg gatgtggcta 231

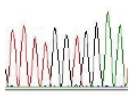

$231 \mathrm{Br}$

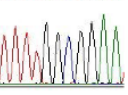

aggetgagtc atctaggagtaaacaagagg ccttcctttgggaggageca atccagggtg tagggggecc agagtgaccaggtgcactag ggaaaaaatgccaggagagg gecaggaaga ggacttgtta gtagcgactcacttctgggc aggcaggecagccagctage cagcctgetg aggettccea agaggggcagagtgctggga tctgggaatccaggaaagga gggaatgggg tggggctaga tgaaaagggataggtgtcca gggagagcctctggctattc ctgggaccag gaagttttca ctaggatacataacactttt tacacactcaccecacceat cectggcttt ctattcatgg aacaacctctctctacaatc cetccagatcttcgcaaata ctgtggacaa tgecegeatc gttetgeagat:gacaatge cegtet.getgetgatgact ttagagtea

Fig. 3.5. The keratin 18 gene is hypermethylated in brain colonizing cells compared to regular breast cancer cells. (a) Expression of keratin 18 in 231 and $231 \mathrm{Br}$ cells after AZA treatment for 72 hours measured by Western blotting assay. GAPDH was used as the loading control. The blots shown are a 
presentation of two independent experiments. (b) Detection of the keratin 18 gene in 231 and $231 \mathrm{Br}$ cells after AZA treatment for 72 hours by PCR. The image shown is a presentation of two independent experiments. (c) Detection of the mRNA level of keratin 18 gene in 231 and $231 \mathrm{Br}$ cells after AZA treatment for 72 hours by real-time PCR. All error bars represent standard deviation (SD), $\mathrm{N}=$ 3 technical replicates, representative of two independent experiments. ${ }^{*} p<0.05$, ${ }^{* *} p<0.01,{ }^{* * *} p<0.001$. (d) Detection and comparison of DNA methylation in the intron 1 region of keratin 18 gene between 231 and 231Br cells. Bold letters indicate the intron 1 region of keratin 8 gene. Inserts show the sequencing chromatogram of bisulfide-converted DNA from 231 and $231 \mathrm{Br}$ cell lines. Sequence in italics shows the Hhal restriction enzyme target site. The DNA sequencing results represents sequencing five positive colonies generated from each pair of primers. (e) Digestion of the genomic DNA isolated from 231 or $231 \mathrm{Br}$ cells with Hhal restriction enzyme. Hhal primer stands for the use of the pair of primers to detect the Hhal digestion by PCR (yielding a $\sim 300 \mathrm{bp}$ PCR product if the DNA was not digested by Hhal, and no such a $\sim 300 \mathrm{bp} \mathrm{PCR}$ product was formed if the DNA was digested by Hhal). Control primer ("cont" in figure) is the pair of primers used as positive control to detect the keratin 18 gene by PCR. The image shown is a presentation of two independent experiments. (f) Expression of DNMT3a and DNMT3b in 231 and $231 \mathrm{Br}$ cells after AZA treatment for 72 hours measured by Western blotting assay. GAPDH was used as the loading control. The blots shown are a presentation of two independent experiments. 
a

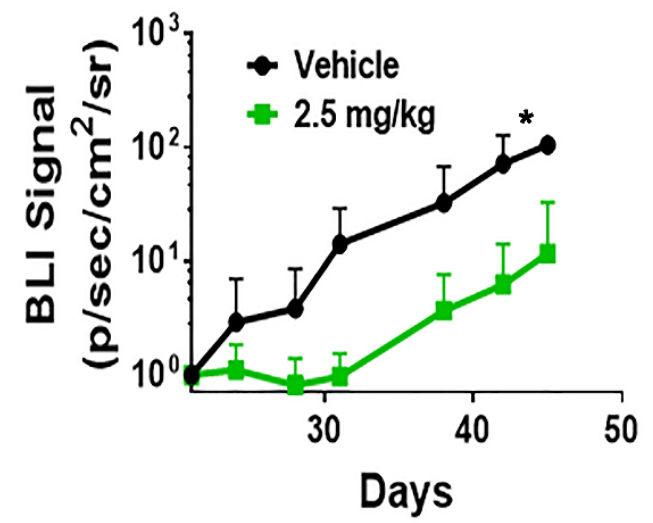

b

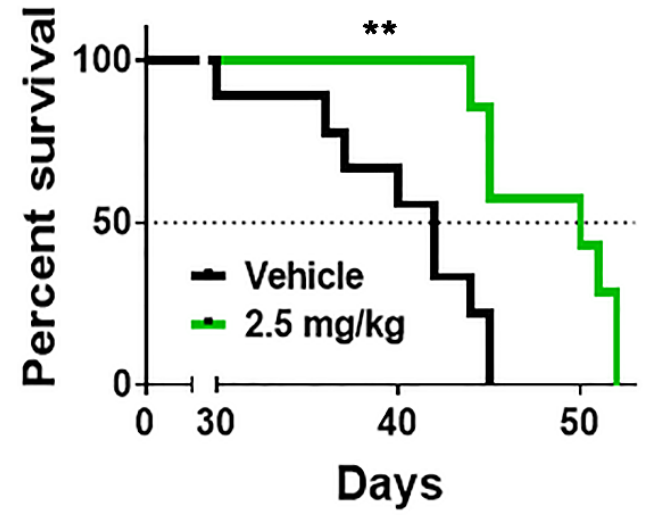

Fig. 3.6. AZA decreases tumor burden and improves survival in mice with brain metastasis of breast cancer. (a) BLI signal versus time in mice with treatment beginning on day 21. Each data point represents the mean plus standard deviation (SD). Mice treated with $2.5 \mathrm{mg} / \mathrm{kg}$ AZA had significantly lower tumor burden $(p=0.0112)$. (b) Kaplan-Meier survival plot of mice starting 21 days after intracardiac injection of 231Br cells. Median survival was 42 days and 50 days, respectively, for vehicle and $2.5 \mathrm{mg} / \mathrm{kg} \mathrm{AZA} \mathrm{(} p=0.0026)$. 


\section{Chapter 4}

\section{Cannabidiol slows the development of breast cancer brain metastases.}

\subsection{Introduction}

Brain metastases from distant primary cancers are the most common type of intracranial mass and typically confer a poor prognosis with limited treatment options. Once diagnosed, patients commonly survive $<2$ years on average [1-3]. Of the primary cancers that metastasize to the brain, advanced breast cancer accounts for roughly $30 \%$ of metastatic CNS lesions [4]. Subtype differences exist, but brain involvement is most common in triple negative and HER2 positive breast cancers [5-7]. Most treatment options for brain metastases only provide palliative care and are rarely curative, but include systemic chemotherapy, radiation therapy, and/or surgical resection if the patient is medically operable [8]. One reason for treatment failure is the blood-brain barrier, which limits the passive diffusion of chemotherapies and actively effluxes many others. The blood-tumor barrier in brain lesions is disrupted, or "leak" [9-19]. However, even in the presence of this leaky vasculature $>90 \%$ of all metastases failed to reach cytotoxic concentrations of systemically delivered chemotherapies in a preclinical model of breast cancer brain metastasis $[10,19]$. For this reason, and due to increased incidence of brain metastasis driven by improved imaging modalities, 
early detection, and enhanced peripheral disease management [20], the development of novel therapeutic strategies to bypass the BTB is critical. Cannabidiol (CBD) is a non-psychoactive, non-toxic constituent of Cannabis Sativa. CBD has been investigated as a potential therapeutic agent in neurodegenerative diseases, pain, anxiety, depression, cancer nausea, inflammation, rheumatoid arthritis, infections, inflammatory bowel disease, and diabetes complications [21-27]. A large body of evidence is being generated in support of $\mathrm{CBD}$ as an anti-cancer agent. The anticancer activity of CBD has been studied extensively in-vitro. A half maximal inhibitory concentration $\left(\mathrm{IC}_{50}\right)$ in a range of $0.67-25 \mu \mathrm{M}$ has been reported in several cancer cell lines [28-30]. Some potential anticancer mechanisms of CBD have also been reported, i.e., reactive oxygen species induced endoplasmic reticular stress, inhibition of epidermal growth factor receptor and insulin growth factor signaling, and downregulation of ID-1 gene expression [29, 31-33].

In addition to CBD's favorable therapeutic activity, CBD has desirable physicochemical properties for brain drug delivery, i.e. a molecular weight of $314.5 \mathrm{~g} / \mathrm{mol}$, a $\log \mathrm{P}$ of 6.5 , two hydrogen bond donors, and two hydrogen bond acceptors. Furthermore, CBD distributes to the brain with a partition coefficient $\left(K_{p}\right.$, brain) of $0.5-4[34,35]$, and is known to inhibit and/or downregulate active efflux transporters leading to enhanced substrate uptake [36-40]. One strategy for overcoming active efflux at the BTB is inhibition of ATP binding cassette proteins, or efflux transporters, such as P-glycoprotein (Pgp; MDR1; ABCB1) and 
breast cancer resistance protein (BCRP; ABCG2) located at the luminal membranes of the microvasculature within the brain. These efflux transporters are known to play a role in the protection of the brain from nearly $90 \%$ of pharmaceutical entities. Inhibition of these proteins has been studied both in-vitro and in-vivo models extensively. Inhibition of efflux transporters by CBD has been studied in-vitro [37, 41], making it an intriguing concurrent therapy for patients suffering from BCBM.

In preclinical metastatic breast cancer models, both immune competent and deficient, CBD significantly decreased breast cancer metastasis to the lungs [30, 33]. In another study, reduced tumor volume was detected in mice treated with CBD [33]. In preclinical glioma models, CBD and THC potentiated temozolomide activity and significantly increased the median survival of glioma bearing mice [44]. In another study, CBD enhanced the radiosensitive of ionizing radiation in vitro and a combination of $\mathrm{THC}, \mathrm{CBD}$ and radiation dramatically reduced intracranially growing glioma [45]. In a recent clinical study, synthetic CBD was able to extend the survival of glioma and breast cancer patients with reduced tumor size and/or circulating tumor cells without any undesired off-target effects [22]. Radiation followed by procarbazine, lomustine, and vincristine (PCV) is a standard of care for high grade-glioma. In a pilot clinical study, CBD enhanced the efficacy of radiation + PCV protocol in high-grade glioma patients [42]. Current clinical studies are underway to look at the efficacy of CBD as a single agent in the treatment of solid tumors [43]. These promising results together with 
CBD's physicochemical properties prompted us to test its efficacy in breast cancer brain metastases.

In this study, a half-maximal inhibitory assay was used to determine if CBD is cable of inhibiting growth and survival of the MDA-MB-231 brain tropic breast cancer cell line $(231 \mathrm{Br})$. The potential for CBD to act as a non-toxic P-gp inhibitor was evaluated via in-situ brain perfusions. Additionally, CBD was evaluated as a single agent in the development of metastatic brain lesions. Lastly, mice bearing 231 $\mathrm{Br}$ metastases were treated with vehicle, $\mathrm{CBD}, \mathrm{PTX}$, or a combination of $\mathrm{CBD}$ and PTX to evaluate the impact of CBD alone and in combination with cytotoxic chemotherapy on tumor burden and overall survival.

\subsection{Methods and Materials}

\section{Materials}

Luciferase transfected brain tropic MDA-MB-231 cells (231Br) were provided by Dr. Patricia Steeg of the National Cancer Institute (Bethesda, MD). Ketamine and xylazine were purchased from Patterson Veterinary (Devens, MA). Cannabidiol was a gift from National Institute on Drug Abuse (Research Triangle Park, NC) via drug supply program. Dulbecco's modified eagle medium (DMEM) and fetal bovine serum (FBS) were purchased from Gibco (Carlsbad, CA). D-luciferin potassium salt was purchased from PerkinElmer (Waltham, MA). Cell culture materials and other laboratory supplies were purchased from Fisher Scientific unless otherwise stated. 


\section{Animals}

All experiments involving animals in this work were approved by the West Virginia University Institutional Animal Care and Use Committee under protocol \# 1604001894. Four to six-week-old athymic nude mice or FVB mice were purchased from Charles River Laboratories (Wilmington, MA) and allowed to acclimatize for three days prior to use in this study. Mice were kept on a 12-hour dark/light cycle with ad libitum access to food and water.

\section{Cell Culture}

Prior to any experiments, $231 \mathrm{Br}$ cells were cultured in DMEM supplemented with $10 \% \mathrm{FBS}$ and maintained at $37^{\circ} \mathrm{C}$ and $5 \% \mathrm{CO}_{2}$.

Half-maximal Inhibitory Assay (IC50).

Exponentially growing $231 \mathrm{Br}$ cells were seeded at a density of 2000 cells per well in a 96-well plate. The next day (24hr), cells were treated with increasing concentrations of CBD ranging from $0.045-100 \mu \mathrm{M}$. After incubation for 48 or 96hr, $10 \mu \mathrm{L}$ of 3-(4,5-dimethylthiazol-2-yl)-2,5-diphenyltetrazolium bromide (MTT) solution $(5 \mathrm{mg} / \mathrm{mL})$ was added to each well. After another $2 \mathrm{hr}$ incubation, media was removed and DMSO was added to dissolve formazan crystals. Cell viability was detected via absorbance at 570nm on a Biotech Epoch plate reader (Winooski, VT) (MTT Assay).

In-situ Brain Perfusion 
Mice were anesthetized deeply with ketamine and xylazine $(100 \mathrm{mg} / \mathrm{kg}$ and 8 $10 \mathrm{mg} / \mathrm{kg}$ respectively). Once unconscious, the thoracic cavity was exposed, the descending aorta was clamped, the right atrium was nicked and an $18 \mathrm{G}$ needle was inserted into the left ventricle. The mouse was then perfused with a physiological buffer solution containing ${ }^{3} \mathrm{H}$-paclitaxel, ${ }^{14} \mathrm{C}$-sucrose, and/or one of three inhibitors (PSC833, elacridar, or cannabidiol) at a flow rate of $5 \mathrm{~mL} / \mathrm{min}$ for three minutes. At the end of the perfusion, the brain was harvested, and the cerebrum, cerebellum, and brain stem were separated. Tissue was dissolved in $2 \mathrm{~mL}$ of solvable overnight at $50^{\circ} \mathrm{C}$. The following morning, the samples were removed and allowed to equilibrate to room temperature. Scintillation fluid, $8 \mathrm{~mL}$, was added and the samples were vortexed. Amounts of ${ }^{3} \mathrm{H}$-paclitaxel and ${ }^{14} \mathrm{C}$ sucrose in cortical regions and perfusion buffer were quantified using a PerkinEImer TriCarb 4910 (Waltham, MA). The unidirectional BBB transfer coefficient $\left(K_{\text {in }}\right)$ was calculated using equation 4.1 as follows:

$$
K_{\text {in }}=\frac{Q_{B r}}{C_{p f} * T}
$$

Where $\mathrm{QBr}_{\mathrm{Br}}$ is the amount of radiotracer in the brain corrected for vascular volume, $\mathrm{C}_{\mathrm{pf}}$ is the amount of radiotracer in the perfusion fluid, and $\mathrm{T}$ is the perfusion time (180 sec) [44-47].

\section{Breast Cancer Brain Metastasis Progression Model}

Athymic female nude mice were randomized into vehicle and cannabidiol groups. Mice received either vehicle $(100 \mu \mathrm{L}, \mathrm{PBS}:$ Ethanol:Tween80, 96:2:2) or cannabidiol $(15 \mathrm{mg} / \mathrm{kg}) 5$ times per week. After pretreatment for 5 days, $1.75 \times 10^{5}$ 
$231 \mathrm{Br}$ cells were injected into the left ventricle as described several times by our laboratory. Tumor growth and cell disposition was monitored twice weekly using an IVIS Spectrum CT 15 minutes following an intraperitoneal dose of D-luciferin potassium salt $(150 \mathrm{mg} / \mathrm{kg})$. Prior to tumor burden analysis, mice were also weighed. As a surrogate for tumor burden, bioluminescent signal in cranial regions was measured. On day 32 , brains were harvested and subsequently stored at $-80^{\circ} \mathrm{C}$ for future analysis.

\section{Cannabidiol Efficacy Study}

Athymic female nude mice were injected with $1.75 \times 10^{5} 231 \mathrm{Br}$ cells through the left cardiac ventricle. Three weeks later, tumor burden was measured using an IVIS Spectrum CT as described above. Mice bearing $231 \mathrm{Br}$ metastases were separated into one of four groups Vehicle, CBD (15mg/kg five times weekly i.p.), PTX (10mg/kg once weekly i.p.), or a combination of CBD and PTX. Mice were collected when humane endpoints became apparent. Brains were again harvested and stored as indicated above.

\section{Data Analysis}

Nonlinear regression analysis was used for $\mathrm{IC}_{50}$ experiments. A one-way ANOVA with subsequent Bonferroni's multiple comparison analysis was used to evaluate in-situ brain perfusion data. A student's t-test was used to determine statistical significance for BLI measurement time points in the prevention studies, and by one-way ANOVA with additional Bonferroni's multiple comparison for efficacy 
study BLI measurements. A log-rank (Mantel-Cox) test was used to analyze median survival data points. All statistical analyses were performed using GraphPad Prism, version 7.00 (GraphPad Software, La Jolla, CA).

\subsection{Results}

Cannabidiol inhibits in-vitro $231 \mathrm{Br}$ cell growth.

To determine the chrematistics of CBD's anticancer activity, we first performed an $\mathrm{IC}_{50}$ analysis using $231 \mathrm{Br}$ cells and MTT assay. CBD's in vitro IC 50 was $13.3 \mu \mathrm{M}$ and $8.5 \mu \mathrm{M}$ at $48 \mathrm{~h}$ and $96 \mathrm{~h}$, respectively (Fig 3.1A). Irrespective of exposure time, inhibition of cell growth was observed above $1 \mu \mathrm{M}$.

$C B D$ increases ${ }^{3} H-P T X$ accumulation during in-situ brain perfusion.

To evaluate the potential of CBD to inhibit P-gp efflux and thereby increase PTX uptake into the brain, we performed in situ brain perfusions in FVB mice and compared ${ }^{3} \mathrm{H}-\mathrm{PTX}$ brain distribution in the presence of PSC833, elacridar, or cannabidiol (Fig 4.1B). The unidirectional uptake rates, $K_{\text {in, for }}$ PTX alone or in the presence of PSC833, elacridar, or cannabidiol were $1.6 \pm 0.1 \times 10^{-4}, 4.9 \pm 0.5$ x $10^{-4}, 58.8 \pm 10.3 \times 10^{-4}$, and $57.1 \pm 5.8 \times 10^{-4} \mathrm{ml} / \mathrm{s} / \mathrm{g}$, respectively. ${ }^{3} \mathrm{H}-\mathrm{PTX}$ uptake was significantly increased in the presence of elacridar and cannabidiol $(p<0.01)$ but was insignificant in the presence of PSC833. The $K_{\text {in }}$ of ${ }^{3} \mathrm{H}-\mathrm{PTX}$ was also insignificant when comparing uptake in the presence of elacridar or cannabidiol but was significant $(p<0.01)$ when comparing uptake in the presence 
of cannabidiol or elacridar to that of PSC833. These data suggest CBD can able to increase the $K_{\text {in }}$ of efflux transporter substrates into the brain.

$C B D$ in the development of breast cancer brain metastasis.

To study the effects of $\mathrm{CBD}$ on the development of $\mathrm{BCBM}$, we used an in vivo mouse model similar to the previous studies. As shown in Fig 4.2A, CBD treatment significantly prevented the weight loss compared to mice receiving vehicle treatment $(p<0.01)$. Vehicle treated mice had a maximum weight loss of $12.5 \pm 4 \%$, while there was no change in the bodyweight of CBD treated mice. The bioluminescent signal within a region of interest circumscribing the cranium was determined in the same mice twice weekly until day 32 . Mice treated with CBD had significantly less tumor burden on day 32 compared to those treated with vehicle as observed in Fig 4.2B $(p<0.05)$. The maximum fold-change on day 32 for vehicle and CBD treated mice were $14.5 \pm 4$ and $6.2 \pm 1$, respectively. These data suggest CBD may be effective in slowing the development of breast cancer brain metastases.

CBD and PTX combination failed to increase survival and decrease tumor burden in a preclinical BCBM model.

To evaluate the efficacy of CBD alone or in combination with PTX, we used our established breast cancer brain metastasis model. Mice were treated with vehicle, CBD, PTX, or a combination of CBD and PTX (C+P) once established brain lesions were observed via BLI on day 21 after inoculation. In all groups, we 
observed that there was no significant difference when comparing BLI intensity (Fig 4.3). Median survival was $38.5,40,38$, and 35 days for the vehicle, PTX, $\mathrm{CBD}$, and $\mathrm{C}+\mathrm{P}$ groups respectively (Fig 4.3B). Of interest, there was no significance among any of the groups compared to vehicle treated mice.

\section{Discussion}

Despite recent advancements in the management of brain metastases from primary breast cancer, treatment outcomes and survival remain dismal. Emerging work indicates that CBD may provide benefits in those suffering from metastatic cancer or brain tumors [22]. In this work, we observed that CBD had a slight positive benefit in the development or progression of metastases, as well as an ability to inhibit P-gp mediate efflux at the BBB through in situ brain perfusion experiments.

In our first experiments, we set out to determine if CBD was capable of killing brain tropic cancer cells in vitro. Through an $\mathrm{IC}_{50}$ analysis, we observed an $\mathrm{IC}_{50}$ of $13.3 \mu \mathrm{M}$ and $8.5 \mu \mathrm{M}$ at $48 \mathrm{~h}$ and 96 hours respectively, suggesting CBD's anticancer activity is concentration dependent and time dependent. The propensity of CBD to induce cell death in multiple cancer cell lines, including prostate, breast, glioma, colon, and gastric adenocarcinoma are displayed in Table $1[28,48]$. Some studies have reported lower IC 50 values in a range of $0.67-3.5 \mu \mathrm{M}$ [49-51]. However, these studies have used different experimental protocols such as using serum free media, frequently replenishing the drug 
containing media, or different methods of quantifying cell death. Results vary depending on the experimental protocol, for example, we have shown that unbound concentrations and activity of anticancer drugs increase with decreasing concentrations FBS in the cell culture media [52]. CBD is a lipophilic molecule (log P of 6.5) with 86-90\% plasma protein binding [53], serum free media will result in $100 \%$ of the drug in the unbound state. Lower CBD IC 50 values reported in the literature can be explained by the modified experimental protocols. Literature values using a similar protocol to the one we have used above confirms the concentration dependent and time dependent anticancer activity of cannabidiol in $231 \mathrm{Br}$ cells (Fig 1A).

To ascertain whether or not CBD could enhance the uptake of substrates that are subject to efflux by P-gp, we performed in situ brain perfusion experiments in the absence or presence of CBD and other known inhibitors. Our data suggest CBD increases the distribution of PTX into brain tissue by up to 37 -fold. To our knowledge, these are the first data set using in situ brain perfusion, though the uptake of P-gp and BCRP substrates in the presence of CBD has been studied in vitro, as well as in ex vivo placental studies [37, 39, 54]. An interesting comparison to our results used the same concentration of CBD $(50 \mu \mathrm{M})$ to demonstrate the increased uptake of mitoxantrone through inhibition of BCRP in vitro [39]. Our results in combination with other reports indicate that CBD is capable of increasing the accumulation of substrates that are subject to active efflux by either P-gp or BCRP. 
In this work, a dose of $15 \mathrm{mg} / \mathrm{kg}$ of CBD was used for in vivo experiments. Previous studies have used $5 \mathrm{mg} / \mathrm{kg}$ and $15 \mathrm{mg} / \mathrm{kg}$ in metastatic breast cancer and glioma models, respectively $[28,31,55]$. Since we are studying breast cancer metastases that are located within the central nervous system (CNS), we chose to use the dose reported in glioma studies. Intraperitoneal injections have been reported to have greater brain CBD concentrations and greater brain/plasma ratios compared to the oral route of administration, supporting our choice of administration route [34]. In humans, six weeks of oral administration of CBD at $10 \mathrm{mg} / \mathrm{kg} /$ day $(\sim 700 \mathrm{mg} /$ day) resulted in a mean plasma concentration between 6 and $11 \mathrm{ng} / \mathrm{mL}(19-35 \mu \mathrm{M})$ [56]. The maximum recommended maintenance dose for Epidiolex is $20 \mathrm{mg} / \mathrm{kg} / \mathrm{day}$, according to the FDA's allometric dosing calculations, the maximum safe dose for mice translates to $246 \mathrm{mg} / \mathrm{kg} / \mathrm{day}$ [57]. Considering these calculations, our dose falls safely within. Brain metastatic models have been used to evaluate the impact of potential drug candidates on the development and/or treatment of breast cancer brain metastases [58]. After pretreating mice for 5 days prior, and continued 5 times weekly thereafter, we saw a decrease in the tumor burden in the CBD treated mice (as suggested by the BLI intensity data, Fig $3 \mathbf{A}$ ) compared to vehicle control. In a similar experiment regarding breast cancer metastasis, CBD $(10 \mathrm{mg} / \mathrm{kg}$, peritumoral injection) in an orthotopic 4T1 breast cancer model decreased lung metastasis [29]. Another group reported that CBD (5mg/kg, i.p.) decreased primary tumor size, as well as the number and size of lung metastases in a 4T1 model of breast cancer [31]. Our data, for the first time in 
the available literature, suggests that CBD is capable of decreasing breast cancer brain metastasis raising potential interests in combinational efficacy studies with other anticancer agents.

In addition, we also evaluated the efficacy of CBD alone and alongside PTX in our preclinical mouse model of breast cancer brain metastases. We observed that $\mathrm{CBD}$ alone or in combination with chemotherapy failed to slow the progression or improve overall survival (Fig $\mathbf{3 A}$ and $3 \mathbf{B}$ ). One potential explanation of these results can be found in previous reports involving inhibition of efflux transporters at the BBB. While many groups have investigated P-gp and BCRP inhibition, translation to humans remains poor. It has been suggested that large increases in CNS penetration are only observed when the percent of BBB efflux inhibition is high, i.e. greater than $90 \%$ [59]. Additionally, the relative concentration of unbound co-administered agents needs to be less than the $\mathrm{K}_{\mathrm{i}}$ or the concentration at which $50 \%$ inhibition of BBB efflux is achieved. Both of these suggest that the implicated limiting factor for clinical BBB efflux in inhibition is low unbound plasma levels of the proposed inhibitor [59].

Further supporting this argument is that the pharmacokinetic parameters of CBD is unlikely to produce an effect. Specifically, CBD is an inhibitor of P-gp [37], but not its substrate [35]. The minimum concentration of CBD required for P-gp

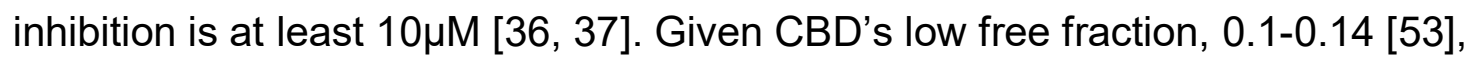
attaining an unbound concentration of $10 \mu \mathrm{M}$ seems unlikely. Given previous 
reports showing that after six weeks of treatment with roughly $700 \mathrm{mg} /$ day, a mean plasma concentration of CBD of $19-35 \mu \mathrm{M}$ was obtained [56]. The corresponding unbound plasma concentrations is $1.9-3.5 \mu \mathrm{M}$, which is well below the desired concentration of efflux inhibition.

Lastly, efflux inhibition depends partly on affinity, $\mathrm{K}_{\mathrm{m}}$, of the inhibitor for the transporter relative to that of the drug substrate for the same transporter. Knowing these parameters may surely provide insight but fell outside the scope of this work.

\section{Conclusions}

In conclusion, the present investigation confirmed CBD's concentration dependent and time dependent anti-cancer activity in $231 \mathrm{Br}$ cell line. CBD was also demonstrated P-gp inhibition at the BBB and able to increase PTX brain uptake in in situ brain perfusion studies. Additionally, CBD was able to decrease the formation of new brain metastases. Lastly, CBD alone and in combination with PTX was unable to improve survival and reduce burden of established brain metastases in a preclinical efficacy model of breast cancer brain metastasis. However, it is important to further explore CBD in different metastatic protocols due to its favorable brain pharmacokinetic properties and beneficial effects regarding chemotherapy induced side effects. 


\section{References}

1. Brogi, E., et al., Breast carcinoma with brain metastases: clinical analysis and immunoprofile on tissue microarrays. Ann Oncol, 2011. 22(12): p. 2597-603. 2. Leone, J.P. and B.A. Leone, Breast cancer brain metastases: the last frontier. Exp Hematol Oncol, 2015. 4: p. 33.

3. Shen, Q., et al., Breast cancer with brain metastases: clinicopathologic features, survival, and paired biomarker analysis. Oncologist, 2015. 20(5): p. 466-73.

4. Wanleenuwat, P. and P. Iwanowski, Metastases to the central nervous system: Molecular basis and clinical considerations. J Neurol Sci, 2020. 412: p. 116755.

5. Heitz, F., et al., Triple-negative and HER2-overexpressing breast cancers exhibit an elevated risk and an earlier occurrence of cerebral metastases. Eur J Cancer, 2009. 45(16): p. 2792-8.

6. Hicks, D.G., et al., Breast cancers with brain metastases are more likely to be estrogen receptor negative, express the basal cytokeratin CK5/6, and overexpress HER2 or EGFR. Am J Surg Pathol, 2006. 30(9): p. 1097-104. 7. Lim, Y.J., et al., Failure patterns according to molecular subtype in patients with invasive breast cancer following postoperative adjuvant radiotherapy: long-term outcomes in contemporary clinical practice. Breast Cancer Res Treat, 2017. 163(3): p. 555-563.

8. Kodack, D.P., et al., Emerging strategies for treating brain metastases from breast cancer. Cancer Cell, 2015. 27(2): p. 163-75. 
9. Lyle, L.T., et al., Alterations in Pericyte Subpopulations Are Associated with Elevated Blood-Tumor Barrier Permeability in Experimental Brain Metastasis of Breast Cancer. Clin Cancer Res, 2016. 22(21): p. 5287-5299.

10. Lockman, P.R., et al., Heterogeneous blood-tumor barrier permeability determines drug efficacy in experimental brain metastases of breast cancer. Clin Cancer Res, 2010. 16(23): p. 5664-78.

11. Sprowls, S.A., et al., Improving CNS Delivery to Brain Metastases by Blood-Tumor Barrier Disruption. Trends Cancer, 2019. 5(8): p. 495-505.

12. Shah, N., et al., Investigational chemotherapy and novel pharmacokinetic mechanisms for the treatment of breast cancer brain metastases. Pharmacol Res, 2018. 132: p. 47-68.

13. Taskar, K.S., et al., Lapatinib distribution in HER2 overexpressing experimental brain metastases of breast cancer. Pharm Res, 2012. 29(3): p. 77081.

14. Adkins, C.E., et al., NKTR-102 Efficacy versus irinotecan in a mouse model of brain metastases of breast cancer. BMC Cancer, 2015. 15: p. 685. 15. Mittapalli, R.K., et al., Paclitaxel-hyaluronic nanoconjugates prolong overall survival in a preclinical brain metastases of breast cancer model. Mol Cancer Ther, 2013. 12(11): p. 2389-99.

16. Mohammad, A.S., et al., Permeability changes and effect of chemotherapy in brain adjacent to tumor in an experimental model of metastatic brain tumor from breast cancer. BMC Cancer, 2018. 18(1): p. 1225. 
17. Adkins, C.E., et al., P-glycoprotein mediated efflux limits substrate and drug uptake in a preclinical brain metastases of breast cancer model. Front Pharmacol, 2013. 4: p. 136.

18. Mittapalli, R.K., et al., Quantitative Fluorescence Microscopy Measures Vascular Pore Size in Primary and Metastatic Brain Tumors. Cancer Res, 2017. 77(2): p. 238-246.

19. Samala, R., et al., Vinorelbine Delivery and Efficacy in the MDA-MB231BR Preclinical Model of Brain Metastases of Breast Cancer. Pharm Res, 2016. 33(12): p. 2904-2919.

20. Smedby, K.E., et al., Brain metastases admissions in Sweden between 1987 and 2006. Br J Cancer, 2009. 101(11): p. 1919-24.

21. Iffland, K. and F. Grotenhermen, An Update on Safety and Side Effects of Cannabidiol: A Review of Clinical Data and Relevant Animal Studies. Cannabis Cannabinoid Res, 2017. 2(1): p. 139-154.

22. Kenyon, J., W. Liu, and A. Dalgleish, Report of Objective Clinical Responses of Cancer Patients to Pharmaceutical-grade Synthetic Cannabidiol. Anticancer Res, 2018. 38(10): p. 5831-5835.

23. Gill., L.L., CBD Goes Mainstream. 2019.

24. Young, S. Marijuana stops child's severe seizures. 2013.

25. Pisanti, S., et al., Cannabidiol: State of the art and new challenges for therapeutic applications. Pharmacol Ther, 2017. 175: p. 133-150.

26. Crippa, J.A., et al., Translational Investigation of the Therapeutic Potential of Cannabidiol (CBD): Toward a New Age. Front Immunol, 2018. 9: p. 2009. 
27. Maida, V. and P.J. Daeninck, $A$ user's guide to cannabinoid therapies in oncology. Curr Oncol, 2016. 23(6): p. 398-406.

28. Ligresti, A., et al., Antitumor activity of plant cannabinoids with emphasis on the effect of cannabidiol on human breast carcinoma. J Pharmacol Exp Ther, 2006. 318(3): p. 1375-87.

29. Elbaz, M., et al., Modulation of the tumor microenvironment and inhibition of EGF/EGFR pathway: novel anti-tumor mechanisms of Cannabidiol in breast cancer. Mol Oncol, 2015. 9(4): p. 906-19.

30. Kis, B., et al., Cannabidiol-from Plant to Human Body: A Promising Bioactive Molecule with Multi-Target Effects in Cancer. Int J Mol Sci, 2019. 20(23).

31. McAllister, S.D., et al., Pathways mediating the effects of cannabidiol on the reduction of breast cancer cell proliferation, invasion, and metastasis. Breast Cancer Res Treat, 2011. 129(1): p. 37-47.

32. McAllister, S.D., et al., Cannabidiol as a novel inhibitor of ld-1 gene expression in aggressive breast cancer cells. Mol Cancer Ther, 2007. 6(11): p. 2921-7.

33. Elbaz, M., et al., Novel role of cannabinoid receptor 2 in inhibiting EGF/EGFR and IGF-I/IGF-IR pathways in breast cancer. Oncotarget, 2017. 8(18): p. 29668-29678.

34. Deiana, S., et al., Plasma and brain pharmacokinetic profile of cannabidiol (CBD), cannabidivarine (CBDV), Delta(9)-tetrahydrocannabivarin (THCV) and cannabigerol (CBG) in rats and mice following oral and intraperitoneal 
administration and CBD action on obsessive-compulsive behaviour.

Psychopharmacology (Berl), 2012. 219(3): p. 859-73.

35. Brzozowska, N., et al., ABC transporters P-gp and Bcrp do not limit the brain uptake of the novel antipsychotic and anticonvulsant drug cannabidiol in mice. PeerJ, 2016. 4: p. e2081.

36. Feinshtein, V., et al., Cannabidiol changes $P$-gp and BCRP expression in trophoblast cell lines. PeerJ, 2013. 1: p. e153.

37. Zhu, H.J., et al., Characterization of P-glycoprotein inhibition by major cannabinoids from marijuana. J Pharmacol Exp Ther, 2006. 317(2): p. 850-7. 38. Holland, M.L., J.D. Allen, and J.C. Arnold, Interaction of plant cannabinoids with the multidrug transporter ABCC1 (MRP1). Eur J Pharmacol, 2008. 591(1-3): p. 128-31.

39. Holland, M.L., et al., The multidrug transporter ABCG2 (BCRP) is inhibited by plant-derived cannabinoids. Br J Pharmacol, 2007. 152(5): p. 815-24.

40. Holland, M.L., et al., The effects of cannabinoids on P-glycoprotein transport and expression in multidrug resistant cells. Biochem Pharmacol, 2006. 71(8): p. 1146-54.

41. Feinshtein, V., et al., Cannabidiol enhances xenobiotic permeability through the human placental barrier by direct inhibition of breast cancer resistance protein: an ex vivo study. Am J Obstet Gynecol, 2013. 209(6): p. 573 e1-573 e15. 
42. Dall'Stella, P.B., et al., Case Report: Clinical Outcome and Image Response of Two Patients With Secondary High-Grade Glioma Treated With Chemoradiation, PCV, and Cannabidiol. Front Oncol, 2018. 8: p. 643.

43. A Study: Pure CBD as Single-agent for Solid Tumor. - Full Text View ClinicalTrials.gov. 2020 02/22]; Available from: https://clinicaltrials.gov/ct2/show/NCT02255292.

44. Smith, Q.R. and Y. Takasato, Kinetics of amino acid transport at the blood-brain barrier studied using an in situ brain perfusion technique. Ann N Y Acad Sci, 1986. 481: p. 186-201.

45. Lockman, P.R., et al., Brain uptake of thiamine-coated nanoparticles. J Control Release, 2003. 93(3): p. 271-82.

46. Lockman, P.R., et al., Brain uptake kinetics of nicotine and cotinine after chronic nicotine exposure. J Pharmacol Exp Ther, 2005. 314(2): p. 636-42.

47. Lockman, P.R., et al., Nicotine exposure does not alter plasma to brain choline transfer. Neurochem Res, 2006. 31(4): p. 503-8.

48. De Petrocellis, L., et al., Non-THC cannabinoids inhibit prostate carcinoma growth in vitro and in vivo: pro-apoptotic effects and underlying mechanisms. $\mathrm{Br} \mathrm{J}$ Pharmacol, 2013. 168(1): p. 79-102.

49. Aviello, G., et al., Chemopreventive effect of the non-psychotropic phytocannabinoid cannabidiol on experimental colon cancer. J Mol Med (Berl), 2012. 90(8): p. 925-34. 
50. McAllister, S.D., L. Soroceanu, and P.Y. Desprez, The Antitumor Activity of Plant-Derived Non-Psychoactive Cannabinoids. J Neuroimmune Pharmacol, 2015. 10(2): p. 255-67.

51. Marcu, J.P., et al., Cannabidiol enhances the inhibitory effects of delta9tetrahydrocannabinol on human glioblastoma cell proliferation and survival. Mol Cancer Ther, 2010. 9(1): p. 180-9.

52. Samala, R., Vinorelbine Distribution to Brain Metastases of Breast Cancer and Factors Limiting in Vivo Efficacy, in Department of Pharmaceutical Sciences, School of Pharmacy. 2011, Texas Tech University Health Sciences Center.

53. Tayo, B., et al., EXPLORATION OF THE POTENTIAL FOR PLASMA PROTEIN BINDING DISPLACEMENT AND DRUG-DRUG INTERACTIONS OF VALPROATE IN COMBINATION WITH CANNABIDIOL, in American Epilepsy Society Annaul Meeting. 2018.

54. Jarocka-Karpowicz, I., et al., Cannabidiol Effects on Phospholipid Metabolism in Keratinocytes from Patients with Psoriasis Vulgaris. Biomolecules, 2020. 10(3).

55. Singer, E., et al., Reactive oxygen species-mediated therapeutic response and resistance in glioblastoma. Cell Death Dis, 2015. 6: p. e1601.

56. Consroe, P., K. Kennedy, and K. Schram, Assay of plasma cannabidiol by capillary gas chromatography/ion trap mass spectroscopy following high-dose repeated daily oral administration in humans. Pharmacol Biochem Behav, 1991. 40(3): p. $517-22$. 
57. (CDER), C.f.D.E.a.R., Guidance for Industry: Estimating the Maximum Safe Starting Dose in Initial Clinical Trials for Therapeutics in Adult Healthy Volunteers. 2005, Food and Drug Administration.

58. Palmieri, D., et al., Profound prevention of experimental brain metastases of breast cancer by temozolomide in an MGMT-dependent manner. Clin Cancer Res, 2014. 20(10): p. 2727-39.

59. Kalvass, J.C., et al., Why clinical modulation of efflux transport at the human blood-brain barrier is unlikely: the ITC evidence-based position. Clin Pharmacol Ther, 2013. 94(1): p. 80-94. 

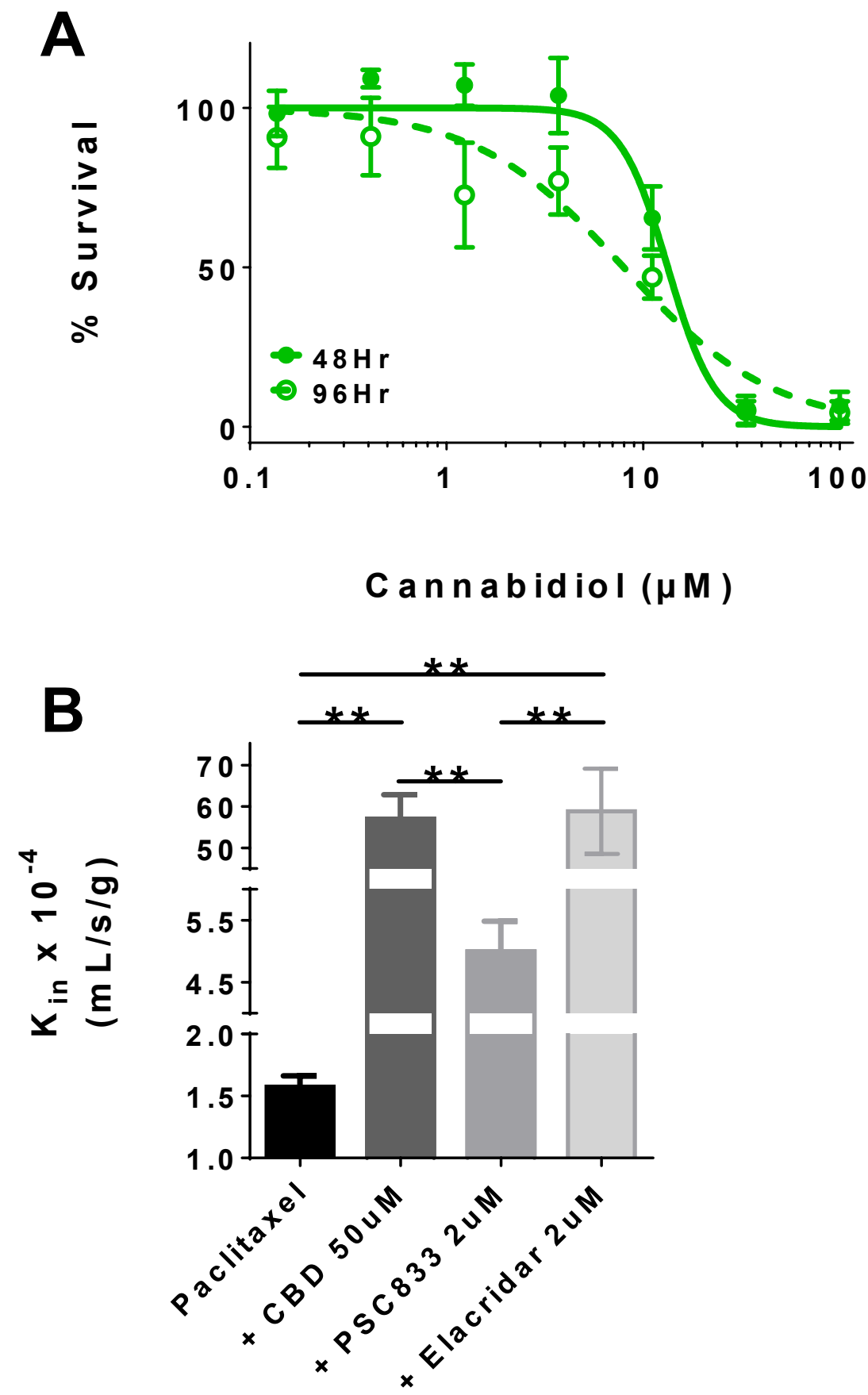

Figure 4.1: Cannabidiol has anticancer activity in the brain tropic MDA-MB231Br cancer cell line and inhibits $\mathrm{P}$-gp at the in-vivo blood-brain barrier. (A) Half-maximal inhibitory assay demonstrating anti-cancer activity of 
cannabidiol at a concentration of $13.33 \mu \mathrm{M}$. (B) Paclitaxel uptake is increased nearly 37 -fold in the presence of cannabidiol at a concentration of $50 \mu \mathrm{M}$ in the physiological perfusion buffer. Data are Mean \pm SEM; $n=3-4$ per group. Oneway ANOVA followed by Bonferroni's Multiple Comparison Test. ${ }^{* *}=\mathrm{P}<0.01$ 

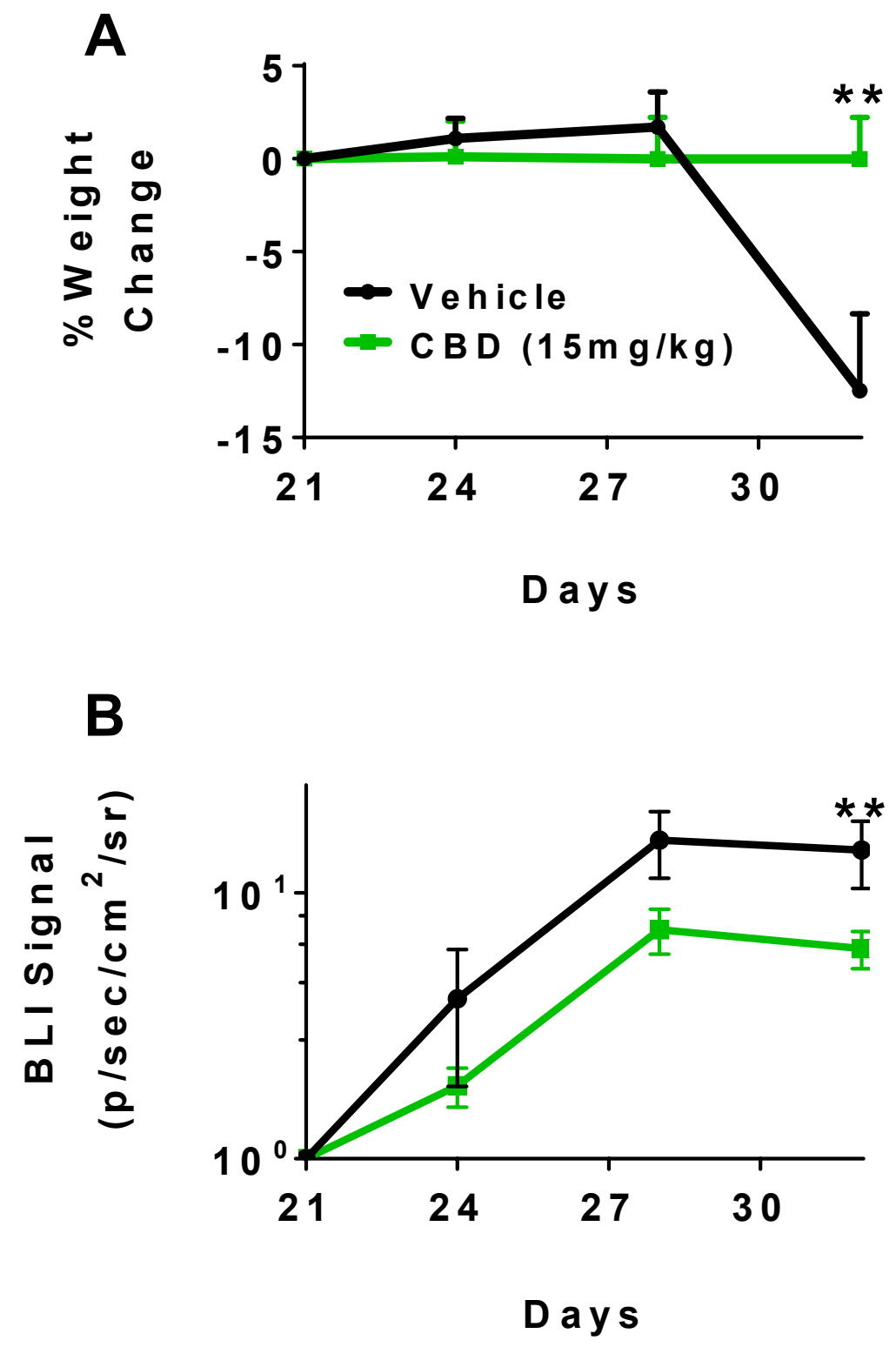

Figure 4.2: Cannabidiol prevents brain metastasis associated weight loss and reduces tumor burden in a preclinical brain metastasis prevention model. (A) Schematic diagram of prevention experiment. (B) Weights of mice treated with cannabidiol $(15 \mathrm{mg} / \mathrm{kg})$ or vehicle control. Mice treated with vehicle lost significantly more weight than mice treated with cannabidiol through the duration of the prevention study. (C) Bioluminescent intensity in mice treated with 
cannabidiol or vehicle. Mice treated with cannabidiol have reduced tumor burden compared to mice treated with vehicle. Data are mean/SD, n= 10/group, unpaired t-test on Day $32,{ }^{* *}=\mathrm{P}<0.01$. 


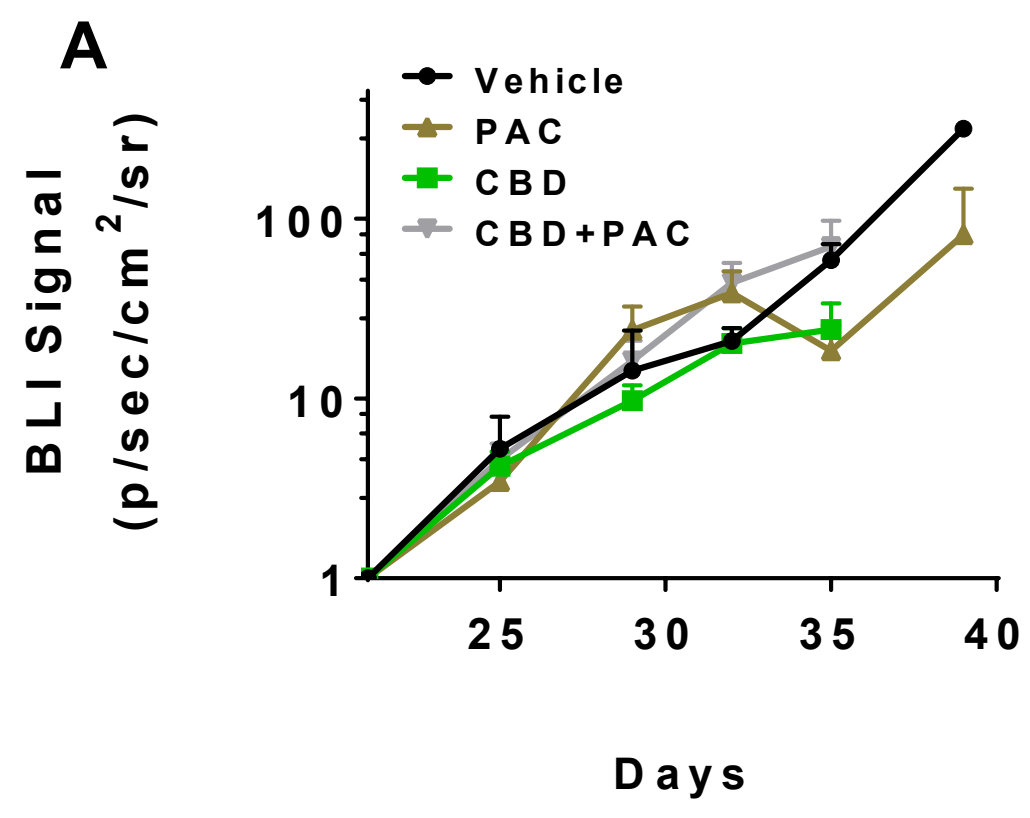

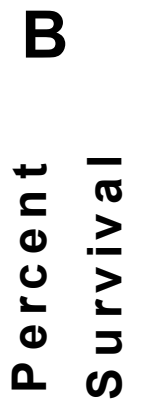

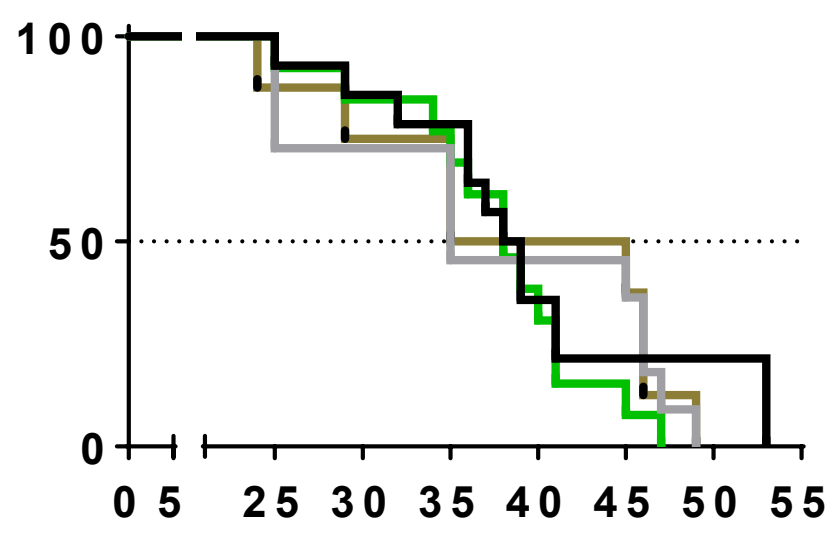

D a ys

Figure 4.3: Cannabidiol in combination with paclitaxel has little to effect on tumor burden and overall survival when compared to vehicle or monotherapy treatments. (A) Timeline schematic of survival study. (B)Bioluminescent signal plotted longitudinally indicating similar tumor burden among all groups. Points represent mean \pm SEM. (C) Kaplan-Meier survival plot 
of mice injected with $231 \mathrm{Br}$ cells and treated with vehicle, cannabidiol $(15 \mathrm{mg} / \mathrm{kg})$, paclitaxel $(10 \mathrm{mg} / \mathrm{kg})$, or a combination of cannabidiol and paclitaxel.

\begin{tabular}{ccccc} 
Cancer Type & Cell line & $\begin{array}{c}\text { Exposure } \\
\text { Time }\end{array}$ & IC 50 & Reference \\
& & & \\
\hline Prostate cancer & DU-145 & $72 \mathrm{~h}$ & $25.3 \mu \mathrm{M}$ & {$[48]$} \\
Prostate cancer & LNCaP & $72 \mathrm{~h}$ & $25.0 \mu \mathrm{M}$ & {$[48]$} \\
Prostate cancer & DU-145 & $96 \mathrm{~h}$ & $20.2 \mu \mathrm{M}$ & {$[28]$} \\
Breast cancer & MCF-7 & $96 \mathrm{~h}$ & $8.2 \mathrm{mM}$ & {$[28]$} \\
Breast cancer & MDA-MB-231 & $96 \mathrm{~h}$ & $10.6 \mu \mathrm{M}$ & {$[28]$} \\
Glioma (Rat) & C6 & $96 \mathrm{~h}$ & $8.5 \mu \mathrm{M}$ & {$[28]$} \\
Colorectal & CaCo-2 & $96 \mathrm{~h}$ & $7.5 \mu \mathrm{M}$ & {$[28]$} \\
carcinoma & & & & \\
Gastric & AGS & $96 \mathrm{~h}$ & $7.5 \mu \mathrm{M}$ & {$[28]$} \\
adenocarcinoma & & & &
\end{tabular}

Table 4.1: Cannabidiol IC $\mathrm{C}_{50}$ in various cancer cell lines. 


\section{Chapter 5}

\section{Radiation increases BTB permeability in a preclinical model of breast cancer brain metastasis.}

\subsection{Introduction}

Breast cancer is the most common cancer diagnosis among women in the United States, affecting nearly one in every eight women, resulting in up to 270,000 new diagnoses each year [1]. Of these women, up to $30 \%$ are at risk for development of brain metastases during their lifetime [2, 3]. After diagnosis with an intracranial lesion survival is poor with only one in five women surviving longer than one year post diagnosis [4]. In triple negative, or basal like, breast cancer (TNBC) up to $30 \%$ of women are likely to develop brain metastases at some point in their lifetime $[5,6]$. Treatment typically includes a combination of radiation, chemotherapy and or surgical resection $[7,8]$. In general drugs for TNBC are limited to cytotoxic chemotherapies, due to the lack of any receptor targets (estrogen, progesterone, and HER2 receptors) [9].

One reason for the overall treatment failure in patients with brain lesions is the presence of the blood-brain barrier (BBB) [10-12]. The BBB is an anatomically unique, physicochemical vascular barrier which forms the interface between blood system and brain $[13,14]$. Under normal physiological conditions the tight junction sealing of BBB endothelia precludes paracellular passive diffusion of 
most solutes into brain parenchyma. While lipophilic molecules may diffuse across the cell membranes, and generally do not rely on paracellular diffusion, active efflux transport pumps, including P-glycoprotein (P-gp, ABCB1), breast cancer resistance protein (BCRP; ABCG2), and multidrug resistance protein-1 (MRP1; ABCC1) [15-19] actively extrude solutes to the luminal side of the BBB. In the context of a brain tumor normal components that surround the BBB, such as astrocytes and neurons are displaced by cancer cells resulting in a leaky vascular barrier, known as the blood-tumor barrier (BTB). While paracellular diffusion is generally higher at the BTB, we have shown previously that it is compromised, the BTB still prevents numerous chemotherapeutics form reaching cytotoxic concentrations in $90 \%$ of all brain metastasis lesions [11].

Current standard of care for brain metastasis of breast cancer usually includes radiation therapy, which may be delivered differently depending on cancer progression and patient status. For a single solitary lesion, the tumor will be resected if operable, and a dose of radiation can be delivered to the resection cavity by (stereotactic radiosurgery) SRS or postoperatively via whole brain radiotherapy (WBRT) to reduce the risk of local and regional recurrence. For patients with a limited number of small intracranial masses $(<3 \mathrm{~cm})$, SRS can be used [20]. Some experts suggest the use of additional, or boost, WBRT following SRS. However, no differences in overall survival have been observed in the data reported in clinical trials comparing the two modalities [21-25]. The use of SRS for 5 or more metastases has been investigated as a stand-alone approach or 
with the use of WBRT in addition to SRS [26-28]. The results from this work are ongoing, but it appears that omitting WBRT may result in increased incidence of distant brain failure and recurrence. Despite the amount of research conducted regarding treatments involving radiation therapy, complications such as neurocognitive decline and local/distant recurrence are unsolved.

While these therapies provide efficacy and may reduce central tumor progression, it has been reported that it may also increase the permeability of the BBB $[10,29]$. However, the timing and magnitude of the BBB and BTB permeability changes are not defined well and remain in some debate in the current literature [10,29]. Several groups have reported permeability changes up to $24 \mathrm{hrs}$ following radiation therapy, while others suggest that any changes occur at later time points. Other reports have not been able to document increases in permeability following radiation treatments [30-38]. Clinically, neurological effects with radiation-induced BBB permeability changes have been segregated into two categories - acute (i.e., initial 24hrs), and those described thereafter, usually weeks to months [39-42].

Based upon the clinical relevance of the therapy, and the relative lack of clarity regarding the effects of radiation on the BBB, we developed a system for brain irradiation in a preclinical model of breast cancer brain metastasis using clinical radiotherapy protocols. Using this model, we quantified the pharmacokinetics of tracer accumulation across the BBB and BTB in a time and size dependent 
fashion. We observed increased permeability of the BTB at both 8 and 24hrs following radiation therapy in our immune-compromised preclinical metastasis model and immune competent model. While there was no BBB disruption in athymic Nu/Nu mice, we did observe increased permeability in immune competent mice. This data suggests that radiation increases the permeability of the BTB and normal BBB with a competent immune system and provides a platform for the study of the mechanism by which this increased permeability occurs.

\subsection{Methods and Materials}

\section{Cell Culture}

Brain tropic, human triple negative breast cancer cells, transfected to express firefly luciferase (MDA-MB-231Br-Luc), were cultured in Dulbecco's Modified Eagle's Medium (DMEM) supplemented with $10 \%$ fetal bovine serum (FBS). MDA-MB-231Br-Luc breast cancer cells were kindly provided by Dr. Patricia Steeg of the National Cancer Institute of Health, Center for Cancer Research.

\section{Development and Optimization of a Half Brain Irradiation Protocol}

To confirm the dose output given by the manufacturer's commissioning of our XenX small animal irradiator (Xtrahl, Suwanee, GA) a Farmer® ionization chamber was placed at a depth of $2 \mathrm{~cm}$ in a solid water commissioning phantom setup and irradiated at $220 \mathrm{KeV}$ and $13.0 \mathrm{~mA}$ for one minute for each of the various conditions required for correction factors as outlined in the Task Group 
61 protocol released by the American Association of Physicists in Medicine (43). The dose output at isocenter, with a source to surface distance (SSD) of $33 \mathrm{~cm}$ and an open radiation field filtered with a $0.15 \mathrm{~mm}$ copper filter was $3.62 \mathrm{~Gy} / \mathrm{min}$. This dose rate was used as a reference to irradiate a set of EBT3 Gafchromic calibration films at doses ranging from 1 to $20 \mathrm{~Gy}$ at a depth of $2 \mathrm{~cm}$ in the same solid water phantom setup. These films were utilized to obtain a standard curve depicting the optical densities of known doses. To determine the dose rate, field homogeneity, and size of our radiation beam collimated with a $10 \times 10 \mathrm{~mm}$ collimator using our custom 3D printed mouse restraint, EBT3 Gafchromic films were irradiated at $0.5 \mathrm{~cm}$ depth in solid water with an additional $1 \mathrm{~cm}$ of solid water below the film to allow for appropriate buildup and backscatter.

\section{EBT3 Gafchromic Film Analysis}

Films were scanned using an Epson (Suwa, Japan) Perfection 4870 flatbed photo scanner in professional mode without color correction at a resolution of 72dpi. Images were analyzed using the red channel on Image J software for all films. Blank, non-irradiated films were also scanned to minimize background for each set of films scanned. All films were scanned at least $24 \mathrm{hrs}$ following irradiation exposure [43]. Optical density (OD) was defined as follows [44]:

$$
n e t O D=\log _{10} \frac{I_{\text {unexp }}}{I_{\text {exp }}}(\text { Equation 5.1) }
$$

To determine dose homogeneity in films irradiated using the $10 \times 10 \mathrm{~mm}$ collimator, the line function was used to determine the dose at each point along the line. For each point OD was calculated. 
Histological Confirmation of Dose Deposition and Absolute Positioning

Naïve female FVB mice were irradiated through the right cranial hemisphere with a single dose of $15.5 \mathrm{~Gy}$ at dose rate of $2.7 \mathrm{~Gy} / \mathrm{min}$. Mice receiving a total dose of 15.5Gy in one fraction is similar to the biological effective dose (BED) of mice receiving a total dose of $30 \mathrm{~Gy}$ in 10 fractions of $3 \mathrm{~Gy}$ with an assumed $\alpha / \beta$ ratio of 10 , accounting for the biological effect being mitotic catastrophe and cell death in MDA-MB-231 breast cancer cells. The equation defining BED can be found below:

$$
B E D=n d\left[1+\frac{d}{\alpha / \beta}\right](\text { Equation 5.2) }
$$

Following treatment mice were anesthetized with ketamine/xylazine (100 and 8 $\mathrm{mg} / \mathrm{kg}$ respectively) before being transcardially perfused with ice-cold 4\% PFA. Mice were decapitated, brains were harvested and then post-fixed overnight in $4 \% \operatorname{PFA}$ at $4^{\circ} \mathrm{C}$. Following fixation, brains were then incubated sequentially in $10 \%, 20 \%$, and $30 \%$ sucrose each for $24 \mathrm{hrs}$. Brains were then co-embedded in 15\% gelatin matrix, 6 brains per matrix, for bulk sectioning. The gelatin matrix was then processed sequentially in $4 \%$ PFA for $24 \mathrm{hrs}, 15 \%$ Sucrose for $24 \mathrm{hrs}$, and $30 \%$ Sucrose for $48 \mathrm{Hrs}$. The block was then trimmed and placed at $-80^{\circ} \mathrm{C}$ for 30 minutes. Brains were then sliced in the coronal plane at a thickness of $30 \mu \mathrm{m}$ on a sliding microtome (HM 450, ThermoFisher Scientific, Waltham, MA) equipped with a $3 \times 3$ freezing stage (BFS-40MPA, Physitemp, Clifton, NJ) at $20^{\circ} \mathrm{C}$. Sections were collected and immuno-stained in 6-well plates containing $0.06 \%$ sodium azide in PBS [45]. 
Sections were immunostained using a standard free-floating section protocol as described $[45,46]$. Briefly, sections were blocked with PBS, methanol, and $30 \%$ hydrogen peroxide (Fisher Scientific, Pittsburgh, PA) and incubated on a shaker for $15 \mathrm{~min}$. Sections were then washed three times and permeabilized for $30 \mathrm{~min}$ on a shaker with $1.83 \%$ lysine (Fisher Scientific, Pittsburgh, PA) in $1 \%$ Triton (Sigma-Aldrich, St. Louis, MO), and 4\% heat-inactivated horse serum (SigmaAldrich, St. Louis, MO). Sections were then incubated for $24 \mathrm{~h}$ with anti-yH2AX (Ser139; 1-500) primary antibody (Cell Signalling Technology, Boston, MA) at room temperature, followed by a $2 \mathrm{~h}$ incubation with the appropriate secondary antibody at room temperature.

\section{Metastatic Brain Tumor Model of Breast Cancer}

MDA-MB-231Br-Luc cells $\left(1.75 \times 10^{5}\right)$ were injected intracardially into the left cardiac ventricle and allowed to develop into metastatic brain lesions for 21 days. Presence of CNS metastases was confirmed by bioluminescent imaging (BLI) on day 21 using the IVIS Spectrum CT imaging system (PerkinEImer, Waltham, MA). D-luciferin potassium salt (150mg/kg; PerkinElmer) was administered intraperitoneally and allowed to circulate for 15 minutes for mice with MDA-MB231Br-Luc metastases before capturing BLI signal. Mice were allowed to progress until substantial tumor burden was observed as indicated by BLI intensity (approximately 4 to 5 weeks).

Radiation Treatments 
Mice were irradiated through a single cranial hemisphere, as to provide the contralateral hemisphere as an internal control for each mouse. Mice received varying doses ranging from 3 to $30 \mathrm{~Gy}$ in fractionation and up to $20 \mathrm{~Gy}$ in a single fraction. All radiotherapy treatments were delivered at a dose rate of $3.01 \mathrm{~Gy} / \mathrm{min}$ using a $10 \mathrm{~mm} \times 10 \mathrm{~mm}$ collimator adjusted to target the right hemisphere. At 8 and $24 \mathrm{hrs}$ following the final irradiation treatments, mice were collected and brain tissue was harvested as described above. Mice were euthanized via exsanguination during the vascular washout period while under deep anesthesia with ketamine/Xylazine (100mg/kg and $8 \mathrm{mg} / \mathrm{kg}$ respectively). Brain tissue was harvested and flash frozen in isopentane $\left(-80^{\circ} \mathrm{C}\right)$ in $<60$ s. Brains were sectioned and mounted on glass slides and stored at $-20^{\circ} \mathrm{C}$ until analyzed via fluorescent microscopy.

\section{Qualitative and Quantitative Fluorescence Imaging}

For all image acquisition, an Upright MVX10 Stereomicroscope (Olympus, Center Valley, PA) equipped with Hamamatsu ORCA Flash4.0 v2 sCMOS camera for fluorescence imaging, a 2x PlanApo (0.5NA) objective, and a DAPI/FITC/RFP/Cy5/Cy7 filter set. The GFP (excitation/band $\lambda$ 470/40nm, emission/band $\lambda 525 / 50 \mathrm{~nm}$ and dichromatic mirror at $\lambda 495 \mathrm{~nm}$ ) filter was used to acquire images confirming half-brain dose deposition with increased $\mathrm{yH} 2 \mathrm{AX}$ signal. Texas Red accumulation in brain metastases was determined by Texas Red sum intensity (SI) per unit area of brain lesion using the RFP filter (excitation/band $\lambda 545 / 25 \mathrm{~nm}$, emission/band $\lambda 605 / 70 \mathrm{~nm}$ and dichromatic mirror 
at $\lambda 565 \mathrm{~nm})$. CellSens image analysis software was used to analyze images and quantitate Texas red accumulation. [47, 48]

\section{Data Analysis}

Differences in permeability between treated and untreated lesions were compared using a student T-test (GraphPad® Prism 7.0, San Diego, CA) and were considered statistically significant at $p<0.05$.

\subsection{RESULTS}

\section{EBT3 Gafchromic Film Dose Response}

The calibration curve for the Gafchromic Film model used is shown in figure 5.1B, and used as a source of reference for dose delivered in all other film analyses. The points correspond to the mean \pm standard deviation determined by use of equation 5.1. In the same graph, corresponding error bars are drawn, but are not visible because they are smaller than the symbols in the figure. The points were fit with a non-linear regression with an $R^{2}$ value of 0.9987 .

Representative images of irradiated films are shown in figure 5.1C-J. As shown, the films have a change in color (or optical density) as the dose of radiation increases.

\section{Half Brain Irradiation Protocol and Histological Verification}

It is important to identify the dose rate of each experimental design in case there are instances of change of dose rate from isocenter under open field conditions. 
To determine the dose output of our experimental design, films were irradiated at a depth of $2 \mathrm{~mm}$ in solid water placed on our custom restraint with the Gafchromic film at isocenter. Images of the film were repeated in triplicate (data not shown). The irradiated field size was consistent with the intentional square field size of $10 \mathrm{~mm} \times 10 \mathrm{~mm}$ measured with calipers (data not shown). The irradiated field was in good agreement with predicted doses and demonstrated both horizontal and vertical beam uniformity as depicted in figure 5.2 . A penumbra of $\sim 0.850 \mathrm{~mm}$ was observed for this treatment field, as defined by the region where the dose drops from $80 \%$ of the max dose deposited to $20 \%$ of the max dose.

To ensure the $10 \mathrm{~mm} \times 10 \mathrm{~mm}$ filed size was accurate and precise for single hemisphere irradiations, individual radiograms were taken of each individual mouse alone and then again with the collimator in place. Images were overlayed using ImageJ at an opacity of $70 \%$ as seen in figure 5.3B. Radiograms were taken under the alignment conditions in figure 5.3A. Our custom 3D printed mouse restraint ensures the placement of the collimated beam for each mouse given the lasers are aligned on the outside border of the right eye (y-orientation) and at the base of the ear (x-orientation) for each mouse. Further confirming targeting of our in-vivo treatments, anti-yH2AX immunofluorescence was used to identify regions exposed to radiation. Figure 5.3C demonstrates the ability to precisely target a single hemisphere in the brain. 
Radiation Therapy Does Not Affect Normal BBB Permeability in Athymic Nu/Nu Mice.

To understand the effects of radiation therapy on normal BBB integrity in our preclinical model of breast cancer brain metastasis, mice were irradiated through the right cranial hemisphere at 3-12Gy in fractionation. Mice were euthanized $24 \mathrm{hrs}$ following the last radiation exposure and the brains were collected, sliced, and analyzed for TxRd accumulation. Compared to untreated hemispheres in mice that were not exposed to radiation of any dose, no significant increase in TxRd accumulation was observed at any dose, indicating that the BBB in athymic $\mathrm{Nu} / \mathrm{Nu}$ mice retains its integrity $24 \mathrm{hrs}$ after radiation therapy (Figure 5.4A-B). The accumulation of TxRd is reported as sum intensity divided by the area of interest $\left(\mathrm{mm}^{2}\right)$ for each area. For mice that did not receive radiation therapy, TxRd accumulation was $4.12 \pm 24$ and in mice that received radiation therapy, the contralateral untreated hemisphere had a value of $4.076 \pm 0.045$. Mice treated to at $3,6,9$, and $12 \mathrm{~Gy}$ had accumulations of $4.17 \pm 0.02,4.15 \pm 0.02,4.08 \pm 0.03$, and $4.10 \pm 0.01$ respectively.

Radiation Therapy Induced BBB Permeability at Low Doses of Radiation Therapy in Immune Competent Mice.

In some patients the immune system elicits an abscopal affect in some patients treated with both radiation therapy and immunotherapy leading to synergistic outcomes. To ascertain the effects of radiation therapy on naïve mice with intact immune function, female FVB mice were irradiated through the right cranial 
hemisphere at doses from 6-30Gy in fraction identical to the fractionation schedule that the Nu/Nu strain mice received. Significant disruption of physiologically normal BBB was observed in mice treated to a total dose of $12 \mathrm{~Gy}$ $(p<0.05)$ and, in mice treated to a total dose of $6 \mathrm{~Gy}$, an obvious increase was observed, although it was not significant (Figure 5.4C,D). At higher doses of 18 and 30Gy, there was no statically significant accumulation of TxRd in irradiated hemispheres compared to the contralateral untreated hemispheres. Means and standard deviations for the contralateral hemispheres, and hemispheres receiving $0,6,12,18$, and $30 \mathrm{~Gy}$ were $3.99 \pm 0.13,4.08 \pm 0.10,4.21 \pm 0.02$, $3.88 \pm 0.02$, and $3.87 \pm 0.01$, respectively.

Radiation Therapy Disrupts the BTB and Increases Permeability at 8 and 24hrs Post Insult.

To understand the effect of radiation therapy on the BTB in our preclinical model of breast cancer brain metastasis, mice were injected with MDA-MB-231Br brain tropic TNBC cells. After substantial tumor burden was measured ( $\sim-5$ weeks) mice underwent radiation treatments to total doses of 6 and 12Gy. Following treatment at 8 and $24 \mathrm{hrs}$ mice were injected with the small (625Da) passive permeability tracer TxRd. After a ten minute circulation period mice were euthanized, brains harvested, and sliced before analysis with a fluorescent microscope. Tumors in the irradiated regions were compared to contralateral, untreated hemispheres for total accumulation of TxRd per lesion size, reported in sum intensity $/ \mathrm{mm}^{2}$. For mice receiving 6 Gy, untreated tumors at 8 and $24 \mathrm{hrs}$ 
following treatment had accumulation of $4.697 \pm 0.272$ and $4.409 \pm 0.284$ respectively, while their treated counterparts had total accumulations of $4.846 \pm 0.600$ and $4.963 \pm 0.777$ at 8 and $24 \mathrm{hrs}$ respectively (Figure 5.5A). For both time points, treated tumors had statistically significant more accumulation of TxRd compared to their untreated counterparts $(p<0.05)$. At the 12Gy dose at the 8 hour time point, untreated and treated lesions had values of $4.239 \pm 0.192$ and $4.389 \pm 0.125$ respectively. The data was not significant (Figure 5.5B). At $24 \mathrm{~h}$ following radiation treatment, values of $4.558 \pm 0.379$ and $4.798 \pm 0.5404$ were determined (Figure 5.5B). Tumors receiving radiation therapy had significantly more accumulation of TxRd at $24 \mathrm{hrs}$ following treatment $(p<0.05)$.

Representative images of an untreated lesion with low permeability to TxRd and a treated lesions with high permeability to TxRd are shown in Figure 5.5C,D.

\subsection{DISCUSSION}

Several studies have investigated the effects of radiation on the BBB or BTB, all reporting different results concerning permeability of brain barriers [49-51]. Additional disparities are observed between reports owing to the non-uniform, clinically dissimilar dosing schemes. In this study we validate a new experimental design using the commercially available XenX Small Animal Irradiator and observed increased BBB permeability to TxRed $24 \mathrm{hrs}$ following a total dose of 12Gy in immune competent animals only. Moreover, we also saw increased permeability of the BTB following low to moderate doses of radiation at 8 and $24 \mathrm{hrs}$ following radiation treatment. 
In this work, first we validated our experimental design through small field radiation dosimetry using a combined ionization chamber and EBT3 Gafchromic® film approach. A similar approach using an equivalent radiation system has been used previously $[52,53]$. Multiple groups have used dose rate measurements in solid water phantoms, cross calibrated with EBT3 films to gauge doses delivered for a particular experimental setup [54]. Herein the dose rate for our small animal irradiator (SAI) at isocenter and an open field was determined to be $3.62 \mathrm{~Gy} / \mathrm{min}$, consistent with dose rates for similar field sizes [52]. The irradiated field demonstrated quality beam uniformity (figure 2) in comparison with our intended field size and had a penumbra, where dose deposition falls from $80 \%$ of the max dose to $20 \%$ of the max dose, measuring $0.850 \mathrm{~mm}$. Measurement and outcomes of beam uniformity and field penumbra for our experimental design are comparable, but vary slightly from others reporting a beam penumbra of $0.40-0.41 \mathrm{~mm}$ [55] using a $10 \times 10 \mathrm{~mm}^{2}$ field. While the beam penumbra is critical in small scale irradiation methodology, the intent of this work was to study the effect of radiation on tumors in a large treatment field consisting of half of the brain. For this purpose, a beam penumbra of $<1 \mathrm{~mm}$ would not deliver substantial dose to the region outside the intended field, nor would it prevent the intended field from receiving a significantly lower dose.

To translate from a dosimetric evaluation of our SAI and its beam characteristics, we transitioned to an in-vivo system. Using naïve female FVB mice and 
immunostaining, we were able to histologically verify successful irradiation of a brain hemisphere by increased $\mathrm{yH} 2 \mathrm{AX}$ signal in the treated hemisphere (figure 3C). The use of anti- $\mathrm{yH} 2 \mathrm{AX}$ staining to ascertain radiation damage, specifically double stranded DNA breaks, and field sizes in in-vivo systems has been established [52, 56, 57].

In order to understand the effects of WBRT on the normal brain and brain tumor vasculature, we modeled clinical dosing patterns to treat and ablate brain metastases. Patients are commonly prescribed a total dose of $30 \mathrm{~Gy}$ over 10 fractions [58, 59]. When fractionation schemes are used, it is critical to understand their translational relevance. One group [60] studied the effects of fractionated radiotherapy on the BBB and BTB in rats. While the dosimetry was well executed, the doses and fractionation patterns do not appear to match what is typically used in patients in the clinic. In a similar study [31], mice were treated with a single fraction of 10Gy. Interestingly, Zarghami et al. [56] limited doses to single fractions, but incorporated the use of a BED equation to demonstrate equivalence to clinical dosing parameters. Of note, changes in fractionation have shown little impact on tumor progression and survival [59].

However, when examining the effects of a treatment on the blood brain barrier, it is important to follow clinical parameters and understand the intent of the treatments. Our experiments were poised to examine the events following a radiation treatment intended to treat brain tumors. Doses outside of what are 
typically used in patients are not necessarily as translationally plausible as studies using methods employed in the clinic. Our findings are presented at low and moderate doses, but were given in the same 3Gy fractions that would be continued to 30Gy in the clinic.

In non-tumor bearing, healthy female Nu/Nu mice, the BBB was unaffected by radiation therapy at doses from $0-12 \mathrm{~Gy}$ in fractions of $3 \mathrm{~Gy}$ at $24 \mathrm{hrs}$ following treatment (figure 4A). Contrary to our results, Wilson et al [30] demonstrated increased normal BBB permeability to a $4.4 \mathrm{kDa}$ FITC dextran at 24 and $48 \mathrm{hrs}$ following radiation. However, this result was following a single exposure to a relatively large, 20Gy dose of radiation. Using the BED equation, this equates to an effective dose that is greater than 1.5 times that of a total dose of $30 \mathrm{~Gy}$ over 10 fractions [61]. Another study using a single dose of 20Gy that used various sized FITC dextran molecules observed increased permeability peaking at $24 \mathrm{hrs}$ post-treatment. However, they observed no increases in normal BBB permeability following a dose of $5 \mathrm{~Gy}$, which is much closer to the single fraction dose we used in our work [37]. The differences in reported measurement of BBB permeability alterations following radiation therapy can be partially attributed to the large heterogeneity in the way the dose was delivered, i.e. high dose vs low dose or single vs multiple fractions.

While our results using athymic nude mice may conflict with reported data, experiments with mice bearing an intact immune system had a different outcome. 
When immune competent female FVB mice were used in the same experiment, we observed a significant increase in normal BBB permeability to TxRed 24hrs following a dose of $12 \mathrm{~Gy}$, as well as an increased, albeit not significant, permeability change $24 \mathrm{hrs}$ following a dose of $6 \mathrm{~Gy}$ (figure $4 \mathrm{C}$ ). It should be noted that in the previously discussed experiments, immune-competent rodent models were used $[30,37]$. These results suggest an active role of the peripheral and CNS immune system in BBB regulation following radiation therapy. Increased cytokine expression has been observed following treatment with radiation [62-64]. Specifically, TNFa, IL1 $\beta$, and IL6 have increased expression, similar to acute periods after neuro-immunological insults [65, 66]. Additionally, at a cerebral blood flow rate of $2 \mathrm{~mL} / \mathrm{min} / \mathrm{g}$ [67], immune cells traversing the cerebrovascular network will be exposed to a substantial dose of radiation, more than likely perturbing an inflammatory response. The damage associated molecular patterns released and innate immune cell cytokine production following radiation therapy could potentially amplify this immune response $[10,68,69]$. All of the underlying inflammatory events following radiation treatments may result in a potential mechanism for BBB disruption in immune competent subjects.

Lastly we set out to determine the effects of WBRT on the vascular system within metastatic brain tumors. Our data indicated increased BTB permeability at both 8 and $24 \mathrm{hrs}$ following treatment with $6 \mathrm{~Gy}$ of radiation in 2 fractions, while after 24hrs we saw increased BTB permeability following a dose of 12Gy in 4 fractions (figure 5). This data is consistent with increased $\mathrm{K}_{\text {trans }}$ values (BBB permeability 
measured clinically) seen in quantitative DCE MRI in irradiated tumors at $24 \mathrm{hrs}$ post-irradiation [70]. Broad beam radiotherapy also displayed increased BTB permeability in treated lesions [71]. Tumor vasculature response has also been studied clinically. In 30 patients and 64 total lesions receiving WBRT or SRS, treatment with radiation increased permeability in initially low leaky tumors [72]. However, in tumors that were already highly permeable, there were no significant increases in permeability. In opposition to what we have observed in this study, there have been observations of no permeability changes measured by MRI gadolinium enhancement [51], though a dose of 20Gy over two fractions was given. While this is different from our study in terms of single fraction dose and fraction number, the BED is similar to that of a completed 30Gy in ten fractions. For a better visualization of how our results align with concluded studies, pertinent data available in the literature for both preclinical and clinical experiments are organized in table 1.

One limitation of this study was the time points that were selected for analysis to determine increased permeability. In the current literature integrity of the BBB and BTB following radiation treatments

\subsection{Conclusions}

In summary, this study was able to provide a means of commissioning for our SAI similar to that detailed by previous work. Additionally we were able to provide a method for targeted, reliable, and reproducible brain irradiation without the need 
for expensive onboard CT equipment. Finally we evaluated permeability at both the BBB and the BTB following radiation therapy with doses of clinical importance. Moving forward, this platform will serve for continued evaluation of brain barriers and their pathophysiology following irradiation, but also to be used as a therapeutic tool in preclinical cancer approaches. Moreover, the difference in normal BBB integrity in different strains of mice with or without an intact immune systems suggests an abscopal-like response to radiation. 


\subsection{REFENCES Table References: $[72,73] \quad$ [60, 74-79]}

1. Siegel, R.L., K.D. Miller, and A. Jemal, Cancer statistics, 2019. CA Cancer J Clin, 2019. 69(1): p. 7-34.

2. DiStefano, A., et al., The natural history of breast cancer patients with brain metastases. Cancer, 1979. 44(5): p. 1913-8.

3. Tsukada, Y., et al., Central nervous system metastasis from breast carcinoma. Autopsy study. Cancer, 1983. 52(12): p. 2349-54.

4. Witzel, I., et al., Breast cancer brain metastases: biology and new clinical perspectives. Breast Cancer Res, 2016. 18(1): p. 8.

5. Lin, N.U., et al., Clinicopathologic features, patterns of recurrence, and survival among women with triple-negative breast cancer in the National Comprehensive Cancer Network. Cancer, 2012. 118(22): p. 5463-72.

6. Pestalozzi, B.C., Brain metastases and subtypes of breast cancer. Ann Oncol, 2009. 20(5): p. 803-5.

7. Lin, N.U., et al., CNS metastases in breast cancer: old challenge, new frontiers. Clin Cancer Res, 2013. 19(23): p. 6404-18.

8. Lin, X. and L.M. DeAngelis, Treatment of Brain Metastases. J Clin Oncol, 2015. 33(30): p. 3475-84.

9. Zangardi, M.L., et al., Sacituzumab for the treatment of triple-negative breast cancer: the poster child of future therapy? Expert Opin Investig Drugs, 2019. 28(2): p. 107-112. 
10. Sprowls, S.A., et al., Improving CNS Delivery to Brain Metastases by Blood-Tumor Barrier Disruption. Trends Cancer, 2019. 5(8): p. 495-505.

11. Lockman, P.R., et al., Heterogeneous blood-tumor barrier permeability determines drug efficacy in experimental brain metastases of breast cancer. Clin Cancer Res, 2010. 16(23): p. 5664-78.

12. Parrish, K.E., J.N. Sarkaria, and W.F. Elmquist, Improving drug delivery to primary and metastatic brain tumors: strategies to overcome the blood-brain barrier. Clin Pharmacol Ther, 2015. 97(4): p. 336-46.

13. Abbott, N.J., Blood-brain barrier structure and function and the challenges for CNS drug delivery. J Inherit Metab Dis, 2013. 36(3): p. 437-49.

14. Wilhelm, I., et al., Role of the blood-brain barrier in the formation of brain metastases. Int J Mol Sci, 2013. 14(1): p. 1383-411.

15. Lagas, J.S., et al., P-glycoprotein, multidrug-resistance associated protein 2, Сур3a, and carboxylesterase affect the oral availability and metabolism of vinorelbine. Mol Pharmacol, 2012. 82(4): p. 636-44.

16. Schinkel, A.H., et al., Disruption of the mouse mdr1a P-glycoprotein gene leads to a deficiency in the blood-brain barrier and to increased sensitivity to drugs. Cell, 1994. 77(4): p. 491-502.

17. de Lange, E.C., et al., BBB transport and P-glycoprotein functionality using MDR1A (-/-) and wild-type mice. Total brain versus microdialysis concentration profiles of rhodamine-123. Pharm Res, 1998. 15(11): p. 1657-65. 
18. Samala, R., et al., Vinorelbine Delivery and Efficacy in the MDA-MB231BR Preclinical Model of Brain Metastases of Breast Cancer. Pharm Res, 2016. 33(12): p. 2904-2919.

19. Adkins, C.E., et al., P-glycoprotein mediated efflux limits substrate and drug uptake in a preclinical brain metastases of breast cancer model. Front Pharmacol, 2013. 4: p. 136.

20. Brown, P.D., et al., Postoperative stereotactic radiosurgery compared with whole brain radiotherapy for resected metastatic brain disease (NCCTG N107C/CEC.3): a multicentre, randomised, controlled, phase 3 trial. Lancet Oncol, 2017. 18(8): p. 1049-1060.

21. Aoyama, H., et al., Stereotactic radiosurgery plus whole-brain radiation therapy vs stereotactic radiosurgery alone for treatment of brain metastases: a randomized controlled trial. JAMA, 2006. 295(21): p. 2483-91.

22. Chang, E.L., et al., Neurocognition in patients with brain metastases treated with radiosurgery or radiosurgery plus whole-brain irradiation: a randomised controlled trial. Lancet Oncol, 2009. 10(11): p. 1037-44.

23. Soffietti, R., et al., A European Organisation for Research and Treatment of Cancer phase III trial of adjuvant whole-brain radiotherapy versus observation in patients with one to three brain metastases from solid tumors after surgical resection or radiosurgery: quality-of-life results. J Clin Oncol, 2013. 31(1): p. 6572. 
24. Kocher, M., et al., Adjuvant whole-brain radiotherapy versus observation after radiosurgery or surgical resection of one to three cerebral metastases: results of the EORTC 22952-26001 study. J Clin Oncol, 2011. 29(2): p. 134-41. 25. Tsao, M.N., et al., Radiotherapeutic and surgical management for newly diagnosed brain metastasis(es): An American Society for Radiation Oncology evidence-based guideline. Pract Radiat Oncol, 2012. 2(3): p. 210-225.

26. Yamamoto, M., et al., Stereotactic radiosurgery for patients with multiple brain metastases (JLGK0901): a multi-institutional prospective observational study. Lancet Oncol, 2014. 15(4): p. 387-95.

27. Yamamoto, M., et al., A Multi-institutional Prospective Observational Study of Stereotactic Radiosurgery for Patients With Multiple Brain Metastases (JLGK0901 Study Update): Irradiation-related Complications and Long-term Maintenance of Mini-Mental State Examination Scores. Int J Radiat Oncol Biol Phys, 2017. 99(1): p. 31-40.

28. Roberge, D., et al., The Future Is Now-Prospective Study of Radiosurgery for More Than 4 Brain Metastases to Start in 2018! Front Oncol, 2018. 8: p. 380. 29. van Vulpen, M., et al., Changes in blood-brain barrier permeability induced by radiotherapy: implications for timing of chemotherapy? (Review). Oncol Rep, 2002. 9(4): p. 683-8.

30. Wilson, C.M., et al., Radiation-induced astrogliosis and blood-brain barrier damage can be abrogated using anti-TNF treatment. Int J Radiat Oncol Biol Phys, 2009. 74(3): p. 934-41. 
31. Deng, Z., et al., Distinct Expression of Various Angiogenesis Factors in Mice Brain After Whole-Brain Irradiation by X-ray. Neurochem Res, 2017. 42(2): p. 625-633.

32. Sandor, N., et al., Low dose cranial irradiation-induced cerebrovascular damage is reversible in mice. PLoS One, 2014. 9(11): p. e112397.

33. Pena, L.A., Z. Fuks, and R.N. Kolesnick, Radiation-induced apoptosis of endothelial cells in the murine central nervous system: protection by fibroblast growth factor and sphingomyelinase deficiency. Cancer Res, 2000. 60(2): p. 3217.

34. Mildenberger, M., et al., An animal model of prophylactic cranial irradiation: histologic effects at acute, early and delayed stages. Int J Radiat Oncol Biol Phys, 1990. 18(5): p. 1051-60.

35. Kyrkanides, S., et al., TNF alpha and IL-1beta mediate intercellular adhesion molecule-1 induction via microglia-astrocyte interaction in CNS radiation injury. J Neuroimmunol, 1999. 95(1-2): p. 95-106.

36. Hong, J.H., et al., Induction of acute phase gene expression by brain irradiation. Int J Radiat Oncol Biol Phys, 1995. 33(3): p. 619-26.

37. Yuan, H., et al., Radiation-induced permeability and leukocyte adhesion in the rat blood-brain barrier: modulation with anti-ICAM-1 antibodies. Brain Res, 2003. 969(1-2): p. 59-69.

38. Nakata, H., et al., Early blood-brain barrier disruption after high-dose single-fraction irradiation in rats. Acta Neurochir (Wien), 1995. 136(1-2): p. 82-6; discussion 86-7. 
39. Baker, D.G. and R.J. Krochak, The response of the microvascular system to radiation: a review. Cancer Invest, 1989. 7(3): p. 287-94.

40. Nordal, R.A. and C.S. Wong, Molecular targets in radiation-induced bloodbrain barrier disruption. Int J Radiat Oncol Biol Phys, 2005. 62(1): p. 279-87.

41. Miot, E., et al., Quantitative magnetic resonance and isotopic imaging: early evaluation of radiation injury to the brain. Int J Radiat Oncol Biol Phys, 1995. 32(1): p. 121-8.

42. Siegal, T. and M.R. Pfeffer, Radiation-induced changes in the profile of spinal cord serotonin, prostaglandin synthesis, and vascular permeability. Int $\mathrm{J}$ Radiat Oncol Biol Phys, 1995. 31(1): p. 57-64.

43. Micke, A., D.F. Lewis, and X. Yu, Multichannel film dosimetry with nonuniformity correction. Med Phys, 2011. 38(5): p. 2523-34.

44. Devic, S., et al., Dosimetric properties of improved GafChromic films for seven different digitizers. Med Phys, 2004. 31(9): p. 2392-401.

45. Nwafor, D.C., et al., Loss of tissue-nonspecific alkaline phosphatase (TNAP) enzyme activity in cerebral microvessels is coupled to persistent neuroinflammation and behavioral deficits in late sepsis. Brain Behav Immun, 2019.

46. Amtul, Z. and J.D. Hepburn, Protein markers of cerebrovascular disruption of neurovascular unit: immunohistochemical and imaging approaches. Rev Neurosci, 2014. 25(4): p. 481-507. 
47. Terrell-Hall, T.B., et al., Trastuzumab distribution in an in-vivo and in-vitro model of brain metastases of breast cancer. Oncotarget, 2017. 8(48): p. 8373483744.

48. Mohammad, A.S., et al., Permeability changes and effect of chemotherapy in brain adjacent to tumor in an experimental model of metastatic brain tumor from breast cancer. BMC Cancer, 2018. 18(1): p. 1225.

49. Yuan, $\mathrm{H}_{\text {., }}$ et al., Effects of fractionated radiation on the brain vasculature in a murine model: blood-brain barrier permeability, astrocyte proliferation, and ultrastructural changes. Int J Radiat Oncol Biol Phys, 2006. 66(3): p. 860-6. 50. Li, Y.Q., et al., Endothelial apoptosis initiates acute blood-brain barrier disruption after ionizing radiation. Cancer Res, 2003. 63(18): p. 5950-6.

51. Murrell, D.H., et al., Evaluating Changes to Blood-Brain Barrier Integrity in Brain Metastasis over Time and after Radiation Treatment. Transl Oncol, 2016. 9(3): p. 219-27.

52. Wong, J., et al., High-resolution, small animal radiation research platform with x-ray tomographic guidance capabilities. Int J Radiat Oncol Biol Phys, 2008. 71(5): p. 1591-9.

53. Ghita, M., et al., Small field dosimetry for the small animal radiotherapy research platform (SARRP). Radiat Oncol, 2017. 12(1): p. 204.

54. Biglin, E.R., et al., Preclinical dosimetry: exploring the use of small animal phantoms. Radiat Oncol, 2019. 14(1): p. 134.

55. Munoz Arango, E.T., J.G. Peixoto, and C.E. de Almeida, Small field dosimetry with a high-resolution 3D scanning water phantom system for the small 
animal radiation research platform SARRP: a geometrical and quantitative study. Phys Med Biol, 2019.

56. Zarghami, N., et al., Half brain irradiation in a murine model of breast cancer brain metastasis: magnetic resonance imaging and histological assessments of dose-response. Radiat Oncol, 2018. 13(1): p. 104.

57. Ford, E.C., et al., Localized CT-guided irradiation inhibits neurogenesis in specific regions of the adult mouse brain. Radiat Res, 2011. 175(6): p. 774-83. 58. Yuan, P. and S.L. Gao, Management of breast cancer brain metastases: Focus on human epidermal growth factor receptor 2-positive breast cancer. Chronic Dis Transl Med, 2017. 3(1): p. 21-32.

59. Tsao, M.N., et al., Whole brain radiotherapy for the treatment of newly diagnosed multiple brain metastases. Cochrane Database Syst Rev, 2012(4): $p$. CD003869.

60. Yusubalieva, G.M., et al., [Blood-brain barrier permeability in healthy rats and rats with experimental C6 glioma after fractionated radiotherapy of the brain]. Zh Vopr Neirokhir Im N N Burdenko, 2015. 79(3): p. 15-26.

61. Fowler, J.F., 21 years of biologically effective dose. Br J Radiol, 2010. 83(991): p. 554-68.

62. Gaber, M.W., et al., Differences in ICAM-1 and TNF-alpha expression between large single fraction and fractionated irradiation in mouse brain. Int $\mathrm{J}$ Radiat Biol, 2003. 79(5): p. 359-66. 
63. Ansari, R., et al., Anti-TNFA (TNF-alpha) treatment abrogates radiationinduced changes in vacular density and tissue oxygenation. Radiat Res, 2007. 167(1): p. 80-6.

64. Kyrkanides, S., et al., Cyclooxygenase-2 modulates brain inflammationrelated gene expression in central nervous system radiation injury. Brain Res Mol Brain Res, 2002. 104(2): p. 159-69.

65. Lambertsen, K.L., K. Biber, and B. Finsen, Inflammatory cytokines in experimental and human stroke. J Cereb Blood Flow Metab, 2012. 32(9): p. 1677-98.

66. Alam, Q., et al., Inflammatory Process in Alzheimer's and Parkinson's Diseases: Central Role of Cytokines. Curr Pharm Des, 2016. 22(5): p. 541-8.

67. Cahill, L.S., et al., Altered cerebral blood flow and cerebrovascular function after voluntary exercise in adult mice. Brain Struct Funct, 2017. 222(8): p. $3395-3405$.

68. Wang, L., et al., Blocking HMGB1 signal pathway protects early radiationinduced lung injury. Int J Clin Exp Pathol, 2015. 8(5): p. 4815-22.

69. Zhou, H., et al., HMGB1 contributes to the irradiation-induced endothelial barrier injury through receptor for advanced glycation endproducts (RAGE). J Cell Physiol, 2018. 233(9): p. 6714-6721.

70. Crowe, W., et al., MRI Evaluation of the effects of Whole Brain Radiotherapy on Breast Cancer Brain Metastasis. Int J Radiat Biol, 2018: p. 1-27. 
71. Bouchet, A., et al., Permeability of Brain Tumor Vessels Induced by Uniform or Spatially Microfractionated Synchrotron Radiation Therapies. Int J Radiat Oncol Biol Phys, 2017. 98(5): p. 1174-1182.

72. Teng, F., et al., Blood-tumor barrier opening changes in brain metastases from pre to one-month post radiation therapy. Radiother Oncol, 2017. 125(1): p. 89-93.

73. Zhou, K., et al., Radiation induces progenitor cell death, microglia activation, and blood-brain barrier damage in the juvenile rat cerebellum. Sci Rep, 2017. 7: p. 46181.

74. Zeng, Y.D., et al., Blood-brain barrier permeability of gefitinib in patients with brain metastases from non-small-cell lung cancer before and during whole brain radiation therapy. Oncotarget, 2015. 6(10): p. 8366-76.

75. Lim, W.H., et al., Does radiation therapy increase gadolinium accumulation in the brain?: Quantitative analysis of T1 shortening using R1 relaxometry in glioblastoma multiforme patients. PLoS One, 2018. 13(2): p. e0192838.

76. Fekete, C. and Z. Liposits, Histamine-immunoreactive neurons of the tuberomammillary nucleus are innervated by alpha-melanocyte stimulating hormone-containing axons. Generation of a new histamine antiserum for ultrastructural studies. Brain Res, 2003. 969(1-2): p. 70-7.

77. d'Avella, D., et al., Radiation-induced blood-brain barrier changes: pathophysiological mechanisms and clinical implications. Acta Neurochir Suppl, 1998. 71: p. 282-4. 
78. d'Avella, D., et al., Quantitative study of blood-brain barrier permeability changes after experimental whole-brain radiation. Neurosurgery, 1992. 30(1): p. 30-4.

79. Fang, L., et al., Whole-brain radiation fails to boost intracerebral gefitinib concentration in patients with brain metastatic non-small cell lung cancer: a selfcontrolled, pilot study. Cancer Chemother Pharmacol, 2015. 76(4): p. 873-7. 

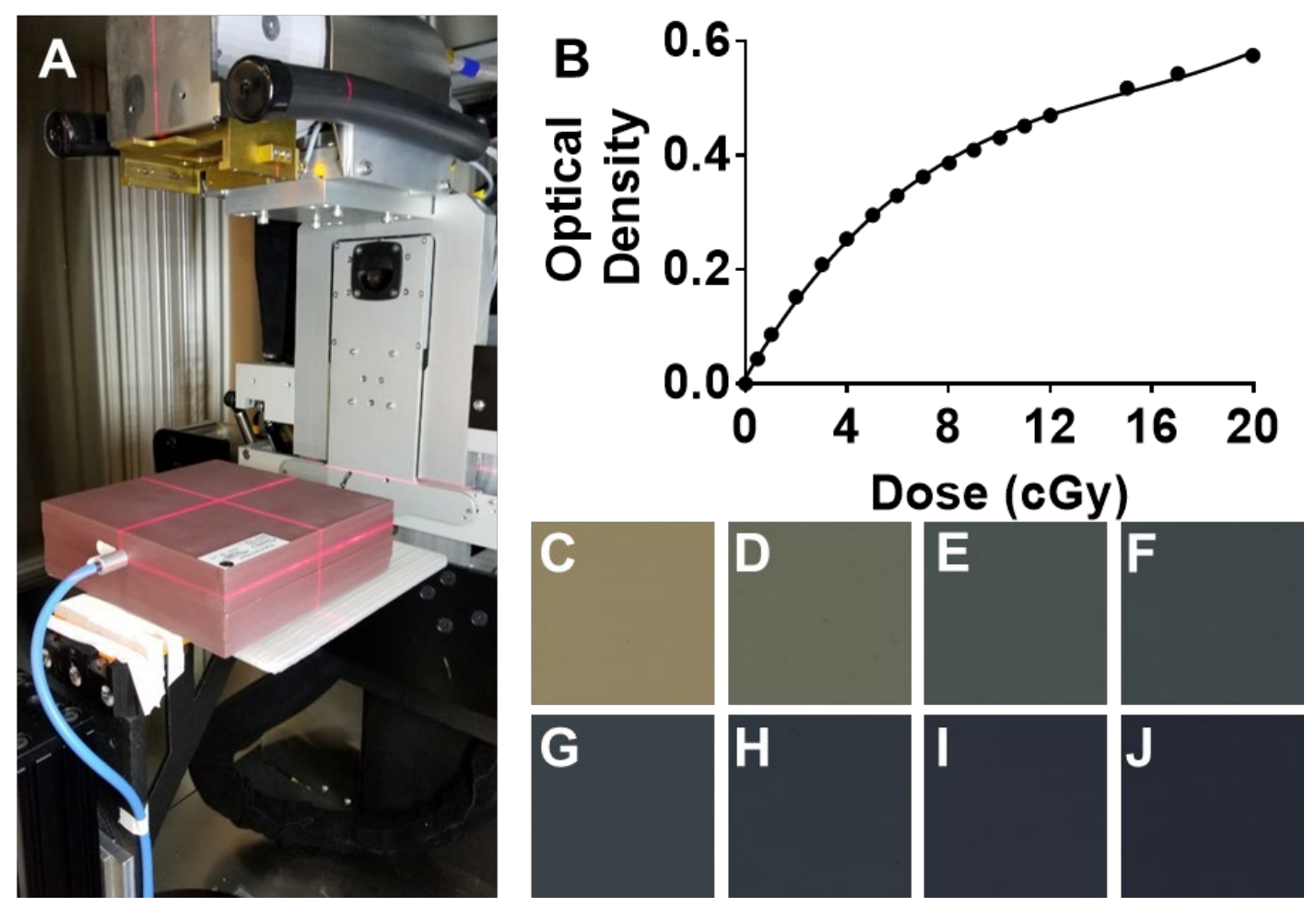

Figure 5.1. Calibration curve at isocenter generated using WVU HSC's Xstrahl Small Animal Irradiator (SAI). (A) Farmer® chamber calibration of WVU HSC's SAI. (B) Calibration curve of Gafchromic EBT3 film generated at isocenter. (C-J) Representative images of film irradiated to doses from 02000cGy. 


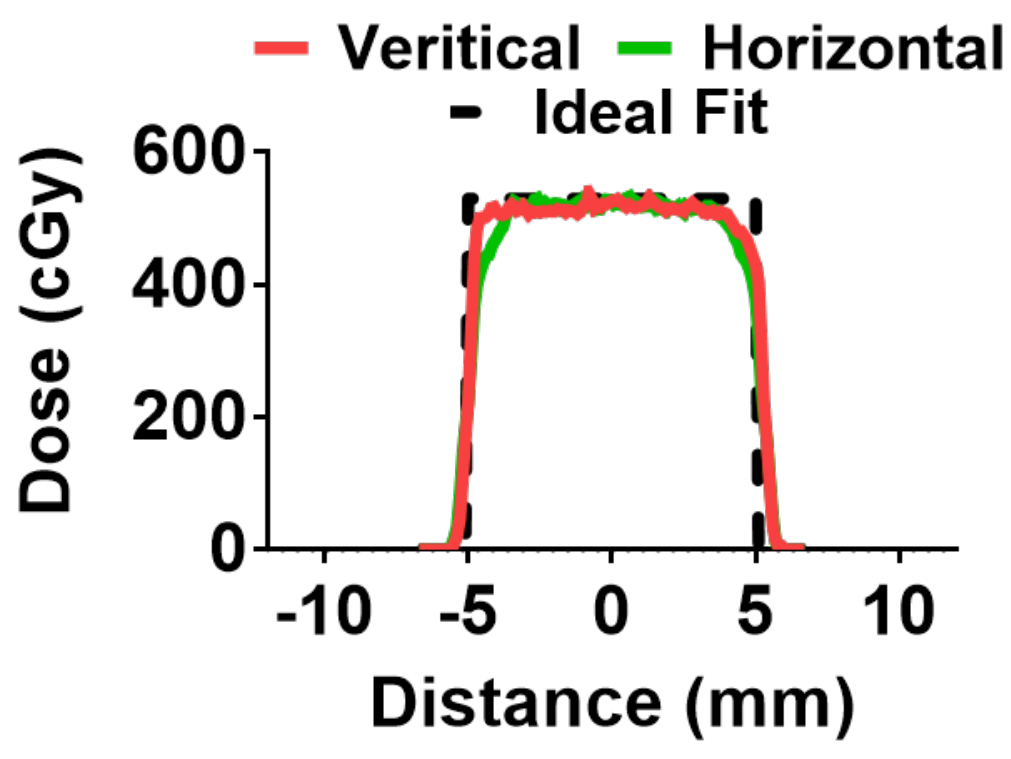

Figure 5.2. Dose homogeneity output of a $10 \times 10 \mathrm{~mm}$ field size irradiated to a target dose of 5.4Gy. The irradiated $10 \times 10 \mathrm{~mm}$ field was uniform in both the horizontal and vertical directions. The penumbra, or the distance between $80 \%$ and $20 \%$ of the max dose was determined to be $0.850 \mathrm{~mm}$.
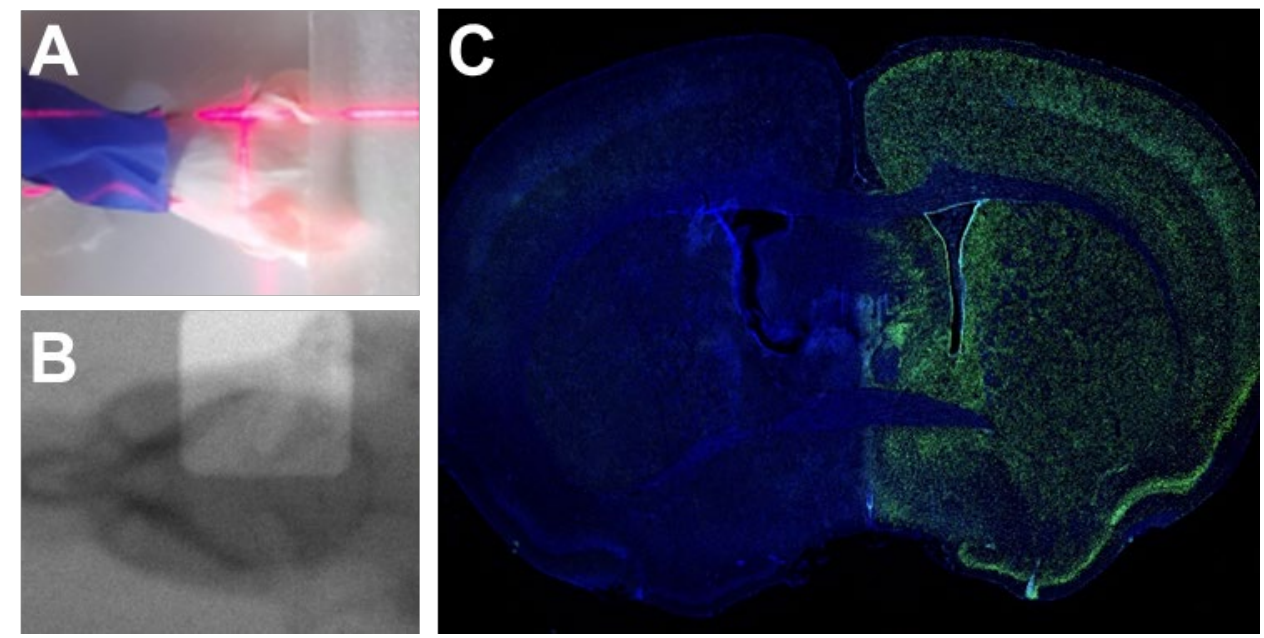

Figure 5.3. Histological verification of half-brain irradiation in an in-vivo system. (A) Representative photographic image of laser alignment on mouse 
providing placement for collimator and (B) dual overlayed radiograms. (C) Representative image of irradiation of FVB mice with a single dose of 15.5Gy through the right cranial hemisphere. Nuclei (Blue) were stained with DAPI. Double stranded DNA breaks (green) are indicated by enhanced $\mathrm{yH} 2 \mathrm{AX}$ signal. 


\section{$\mathrm{Nu} / \mathrm{Nu}$}
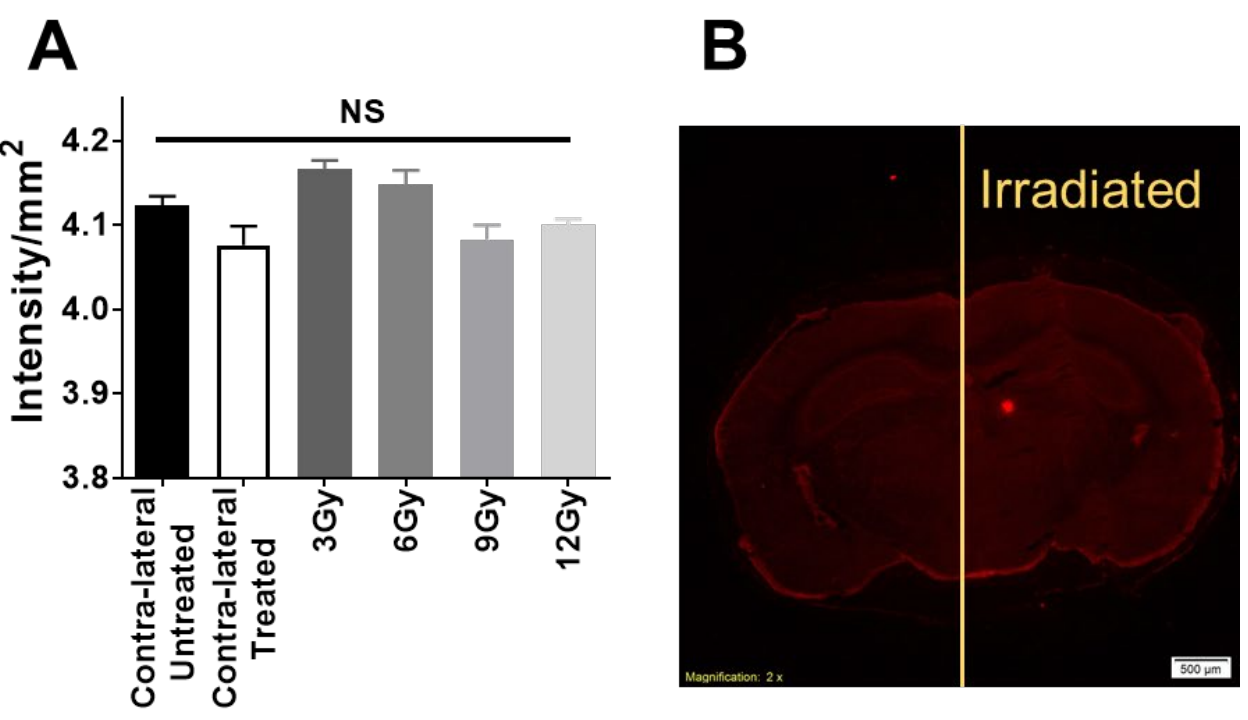

FVB
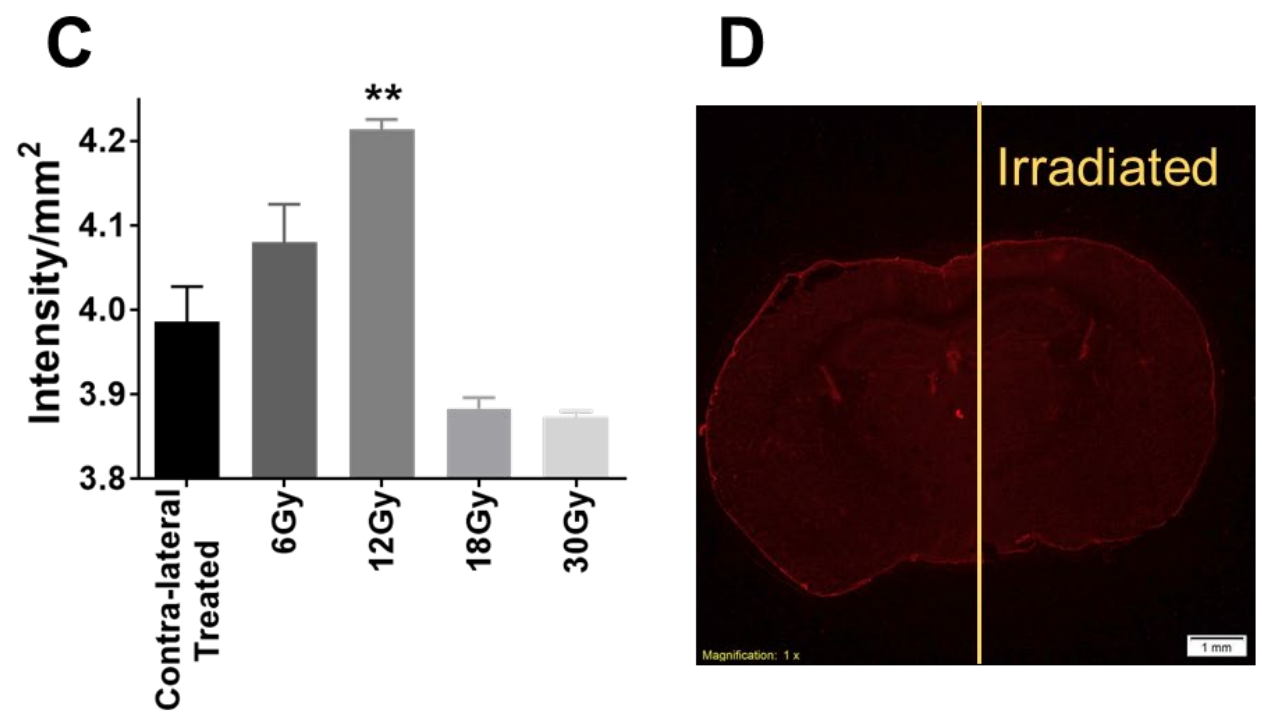

Fig 5.4. The BBB remains intact in athymic Nu/Nu mice but is disrupted at an intermediate dose in immune competent FVB mice. (A) Athymic nude mice treated with daily fractions of $3 G y$ showed no significant difference in normal BBB permeability to Texas Red at 24 hours following radiotherapy. (B) Representative image of a Nu/Nu mouse treated with radiotherapy through the 
right cranial hemisphere. (C) Immune competent FVB mice showed no significant difference in BBB permeability to Texas Red, except following a total dose of 12Gy given in 4 fractions. (D) Representative image of a FVB mouse treated with radiotherapy through the right cranial hemisphere. 


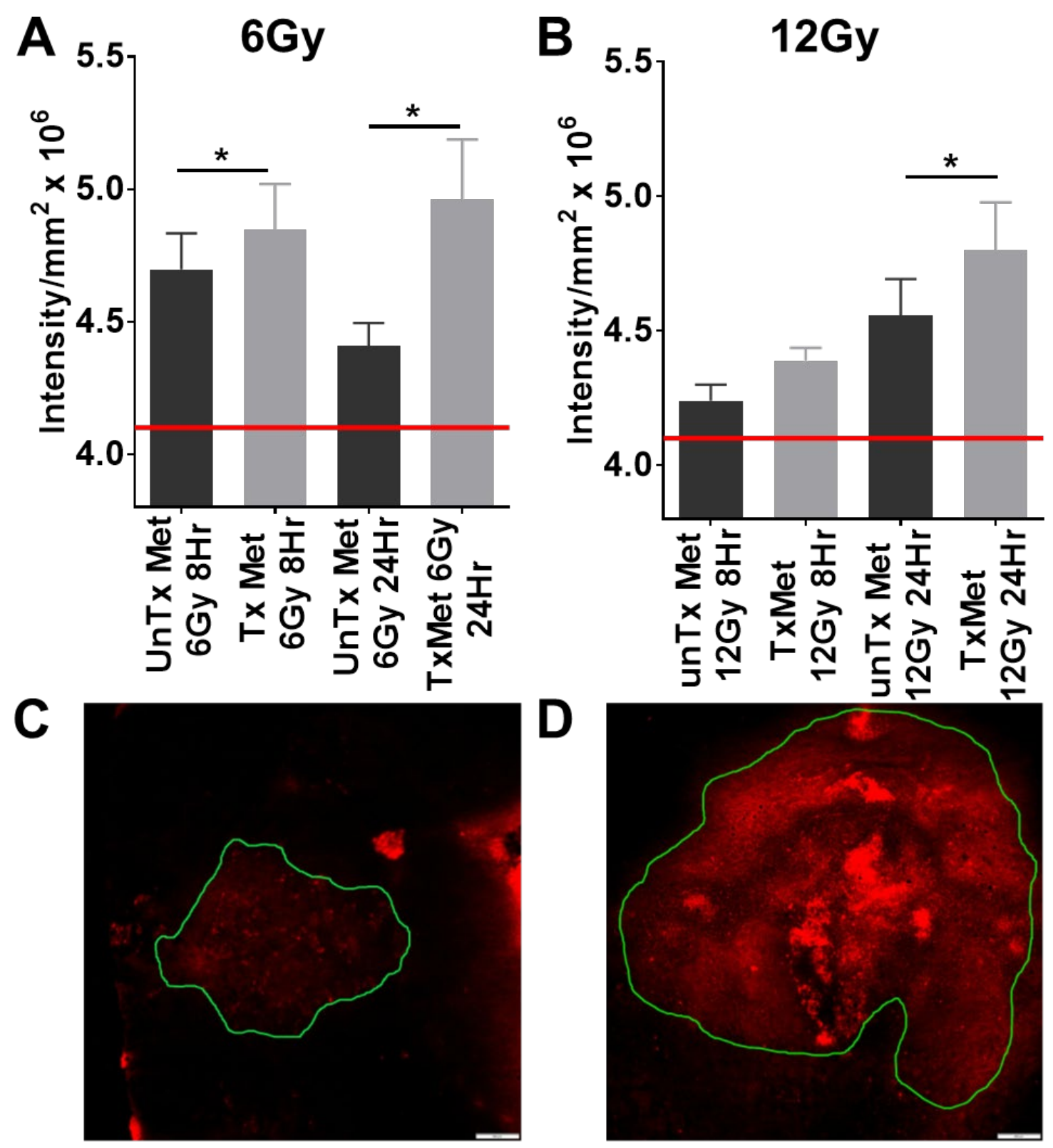

Fig 5.5. Permeability of metastatic brain lesions increases in a time and dose dependent manner following half-brain irradiation. (A) BTB permeability is significantly increased at both 8 and 24 hours following $6 G y$ $(p<0.05, n=13)$ in metastatic tumors in the portion of the brain receiving radiation treatment. In the mice treated with 12Gy of radiation a significant increase in BTB permeability to Texas Red was only seen at 24 hours post treatment $(p<0.05$, 
$n=12-18)$. (C-D) Representative images of an untreated metastatic brain lesion and a lesion that was in the radiation field. 


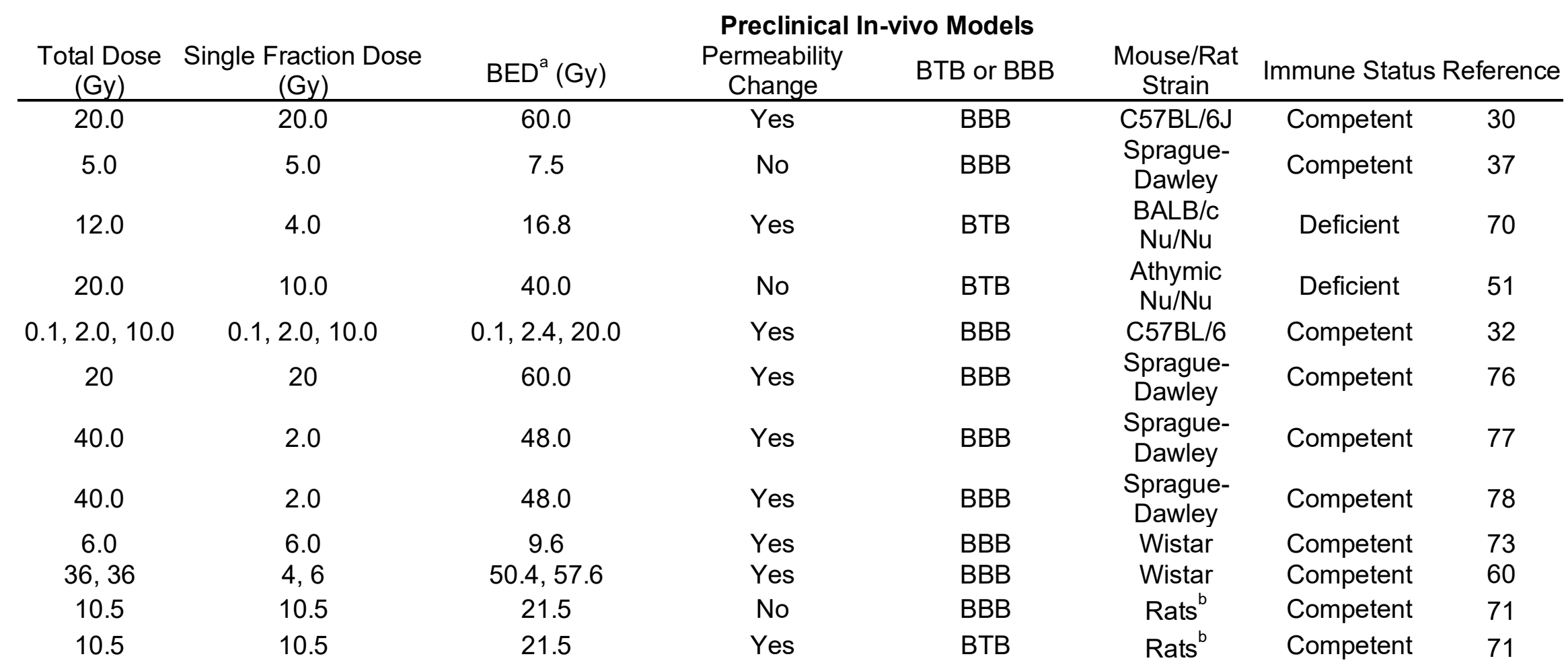

${ }^{a}$ Biological effective dose (BED) was calculated using the parameters given in reference cited, with an assumed $\alpha / \beta$ ratio of 10 .

${ }^{\mathrm{b}}$ Strain of rat unmentioned. The F98 glioma model is commonly used in Fisher rats, which are immunocompetent.

Table 5.1. Comparison of dose, BED, and permeability changes among literature reports investigating the BBB and radiation therapy in preclinical models. 


\begin{tabular}{|c|c|c|c|c|c|}
\hline Total Dose (Gy) & $\begin{array}{l}\text { Single Fraction Dose } \\
\qquad(\mathrm{Gy})\end{array}$ & $\mathrm{BED}^{\mathrm{c}}(\mathrm{Gy})$ & Permeability Change & BTB or BBB & Reference \\
\hline 30.0 & 3.0 & 39.0 & Yes & BTB & 72 \\
\hline 37.5 & 2.5 & 46.9 & Yes & BTB & 72 \\
\hline $10.0,20.0,30.0,40.0$ & 2.0 & $12.0,24.0,36.0,48.0$ & Yes & BTB & $74^{a}$ \\
\hline $57.0 \pm 7.2$ & $\mathrm{Na}^{\mathrm{b}}$ & $\mathrm{Na}^{\mathrm{b}}$ & Yes & BBB & 75 \\
\hline 30Gy & 3.0 & 39.0 & No & BTB & 79 \\
\hline
\end{tabular}

${ }^{a}$ Doses were in sequential order over the normal treatment schedule. Plasma and CSF samples were taken after each $10 \mathrm{~Gy}$ segment.

${ }^{b}$ Data not given in manuscript.

${ }^{c}$ Biological effective dose (BED) was calculated using the parameters given in reference cited, with an assumed $\alpha / \beta$ ratio of 10 .

Table 5.2. Comparison of dose, BED, and permeability changes among literature reports investigating the BBB and radiation therapy in clinical patients. 


\section{Chapter 6}

\section{A Review of Mathematics Determining Solute Uptake at the Blood-Brain Barrier in Normal and Pathological Conditions}

\subsection{The Blood-Brain Barrier}

The blood-brain barrier (BBB; Figure 1) is the tightly regulated interface of the brain and its microvascular system composed of endothelial cells (ECs), a basement membrane, pericytes, astrocytes, neurons, and microglia. Collectively, these structures cooperate as a highly selective functional barrier capable of regulating the distribution of molecules to brain parenchyma. Claudins, occludins, and junction adhesion molecules (JAMs) form an extensive paracellular barrier between ECs to small molecules, proteins and cells [1]. The ECs at the BBB also exhibit lower rates of transcytosis as a result of non-fenestrated vessels and decreased caveolin-mediated vesicle trafficking compared to the peripheral vascular system [2]. Together, pericytes and ECs secrete an extracellular matrix that sur-rounds the blood vessels within the brain and forms the basement membrane in which pericytes and astrocytic end-feet become embedded. Astrocytes form the outer layer of basement membrane. The basement membrane serves to facilitate essential intercellular signaling while simultaneously promoting the selective distribution of molecules into the brain parenchyma [3]. The unique characteristics of the BBB impart decreased 
permea-bility of solutes in comparison to other vascular networks throughout the periph-ery.

The primary functions of the BBB are to maintain homeostasis of the brain microen-vironment and provide neuroprotection. The BBB preserves the brain microenvironment with influx and efflux transporters. Examples of these include the OCT1, OCT 2, LAT1, and OAT solute carrier protein transporters, which have been suggested to facilitate drug influx at the BBB $[4,5]$, and the P-glycoprotein efflux transporter, which minimizes the passage of many lipophilic solutes from reaching the abluminal membrane of the brain capillary network [6].

Dysregulation of the BBB affects cell signaling, immune cell traf-ficking, and potential neuronal damage [7]. Selective permeability of the BBB is essential for maintaining central nervous system health, but becomes an obstacle to therapeutic drug distribution into the brain to treat neurological disorders or malignancies of the CNS [8].

\subsection{Mapping Drug Kinetics at the Blood-Brain Barrier}

Mathematical determination of solute permeability rates across the BBB has not dramatically changed since Patlak et al [9]. described their, at the time, novel model. In their work, the authors proposed a two-compartment model in which influx across the BBB is an irreversible, unidirectional process during the experimental time frame. Model experiments include multiple blood, or plasma, measurements following an intravenous bolus tracer dose across the duration of the experimental time frame from the same subject. Tracer concentration in brain 
is also obtained at multiple timepoints assumed to be in the linear range of uptake for a given tracer. Plotting the ratio of concentration of tracer in brain $(\mathrm{CBr})$ at time $\mathrm{t}$ to concentration of tracer in plasma $(\mathrm{Cpl})$ at time $\mathrm{t}$ versus the total exposure of the animal to a given tracer from time 0 to time $t$ produces a linear plot as long as the experiment is performed in the range of linear uptake of the given tracer. Regressing these data produces a line with slope of Kin, or the unidirectional transfer constant for the tracer used and y intercept representing the cerebral vascular volume of the test subject measured in units of volume/time/mass, typically as $\mathrm{mL} / \mathrm{s} / \mathrm{g}$. The expression for movement of solute from the brain capillary network and into the extravascular compartments is given in Equation (1) [9]:

$$
C_{B r}=K_{i n} \int_{0}^{t} C_{p l} d \tau+\left(V_{0}+V_{p l}\right) C_{p l}
$$

where $\mathrm{CBr}$ (tracer/g of brain) is the concentration of tracer in brain, $\mathrm{Cpl}$ (tracer/mL) is the concentration of tracer in plasma, $\mathrm{V} 0(\mathrm{~mL} / \mathrm{g})$ and $\mathrm{Vpl}(\mathrm{mL} / \mathrm{g})$ constitute the total tracer concentrations within the brain capillary network, and Kin $(\mathrm{mL} / \mathrm{s} / \mathrm{g})$ is the unidirectional transfer constant for a given solute.

To simplify the kinetic expressions and complement the data from the method above, Takasato and colleagues applied the principles above and created an in situ brain perfusion technique in which the concentration of tracer in the plasma, or in this case the perfusion buffer, remains constant. The in situ brain perfusion technique has several advantages over many traditionally applied barrier integrity 
protocols. The most striking difference that sets the perfusion technique apart from other methodologies is the ability of the researcher to alter the buffer used to study active transport, protein binding, and a host of other interesting interactions at the BBB. Addition of increasing unlabeled substrate in combination with a constant concentration of radiolabeled substrate can provide insight to the Michaelis-Menten kinetics for a particular substrate-transporter relationship [10]. Additionally, adding serum proteins, adding known inhibitors of efflux transporters [11], or by cooling the perfusion below $37^{\circ} \mathrm{C}$ provides the researchers the ability to study the effects of plasma protein binding [12], the affinity of a given substrate for a particular efflux transporter [13], and the impact of temperature [12] on nutrient transport at the BBB. Other notable advantages include avoidance of extracranial metabolism of the solute of interest, less extensive animal surgery, and the possibility to study permeability coefficients over a 104-fold range. The perfusion technique does not replace the intravenous injection technique, but complements the data obtained. The pharmacokinetic expression used to determine tracer uptake in the in situ brain perfusion technique is as follows in equation 2 [14]:

$$
Q_{B r} / C_{p f}=K_{i n} T+V_{0}(2)
$$

where $\mathrm{QBr}$ (tracer/g of brain) is the final concentration of solute in the brain, Cpf (tracer $/ \mathrm{mL}$ ) is the tracer concentration is the perfusion buffer, $\mathrm{T}(\mathrm{s})$ is perfusion time, $\mathrm{V} 0(\mathrm{~mL} / \mathrm{g})$ is the intercept of the vascular marker used in the experiment 
(also known as the vascular volume), and $\mathrm{Kin}(\mathrm{mL} / \mathrm{s} / \mathrm{g})$ is the unidirectional transfer constant, obtained from the regressed slope of the brain distribution volume versus time graph.

Data obtained from either of these techniques have been used to make predictive models to determine how fast a novel or understudied solute may permeate across the BBB. A handful of physiochemical properties have been determined to most notably influence BBB permeability: molecular weight, hydrogen bond donors, total polar surface area, and lipophilicity, or LogP. A good agreement exists when plotting the literature, or experimentally verified, permeability coefficients against some variation of the lipophilicity of the same solute. A variety of mathematical alterations to $\log P$ and the use of physiochemical properties in combination with LogP have been used to form predictive estimates of uptake of a multitude of solutes. Figure 2 demonstrates the relative correlation between LogPS and Log (oil/water partition coefficient : $\sqrt{ } \mathrm{MW}$ ). Using the slope from the linear regressed line and the physiochemical properties of a novel substrate, a theoretical or predicted permeability coefficient can be determined.

\subsection{Active Efflux at the Blood-Brain Barrier}

The BBB dynamically regulates homeostasis and protects the brain from exposure to endogenous entities, toxic drugs and other xenobiotic substances. Multidrug transporters present at the luminal surface of the BBB contribute towards protection by controlling drug distribution and elimination from the brain by ATP-mediated efflux. A majority of these efflux transporters belong to the ATP 
binding cassette $(A B C)$ superfamily and demonstrate broad affinity for many clinically used drugs based on structure and chemistry of the molecule. Previous studies demonstrate differential expression of several types of $A B C$ transporters at the BBB including P-glycoprotein (P-gp, MDR1, ABCB1), breast cancer resistance protein (BCRP, ABCG2), multidrug resistance protein (MRP1-6, $A B C C 1-6)$ and the organic anion transporter (OAT3) $[13,15]$. Amongst these, the most clinically relevant $A B C$ transporters implicated in prohibiting drug delivery to the brain are P-gp and BCRP. These transporters are responsible for limiting brain access to a wide variety of substrates as a result of extensive expression at the BBB and blood-cerebrospinal fluid barrier (BCSFB) [16, 17]. Recent studies demonstrate that these transporters have overlapping affinities for certain substrates which might lead to higher inhibitory effect to drug permeability as opposed to that observed for the individual transporters [18].

The kinetics of efflux can be determined using either of two approaches. Performing in situ brain perfusions as described above to a point of steady state, or to a point where the ratio of tracer in brain to the quantity of tracer in blood does not increase further with time, enables the use of Equation (3) [10].

$$
V_{B r}=\frac{K_{\text {in }}}{K_{\text {out }}}
$$

where $\mathrm{Vbr}(\mathrm{mL} / \mathrm{g})$ is the volume of distribution, or the ratio between tracer quantity in brain and blood, Kin $(\mathrm{mL} / \mathrm{s} / \mathrm{g})$ is the unidirectional transfer constant reflecting the rate at which a substance crosses the brain capillary barrier into the 
parenchyma, and Kout (s-1) is the rate of efflux of the same solute. A second way to measure the efflux constant is to use a modified in situ brain perfusion in which the brain is preloaded with the solute of interest for a nominal time, and then perfused with tracer-free perfusate for multiple durations. The brain/perfusate ratio can then plotted against time. Kout $(s-1)$ can be determined from these data using the following expression in equation 4 [10]:

$$
K_{\text {out }}=\ln 2 / t_{1} / 2
$$

where Kout $(s-1)$ is the rate of solute efflux from the brain capillary system, and $\mathrm{t} 1 / 2$ is the half-life of linear regressed line on the brain/perfusate ratio versus time plot. A similar efflux constant can be determined using either expression so long as the experiments are performed correctly. The in situ brain perfusion technique is a sensitive, effective method that can be used to determine efflux kinetics as described above. Previously, the efflux of thiamine at the BBB was determined using both Equations (3) and (4) [10]. Thiamine efflux did not significantly vary between different brain regions. Interestingly, using predictive models can provide an estimate of Kin as described above. When actual measurements of Kin differ dramatically from predictive models, these compounds are typically subject to efflux. Additionally, in relation to Figure 2, compounds that are effluxed at the BBB typically fall below the linear regressed line indicating that something is preventing them from passing through the BBB as they should based on their physiochemical properties. 
Bart et al. used the parameter of distribution volume (DV) to quantify the efflux of P-gp substrate [11C]verapamil $[19,20]$. The efflux of radiolabeled verapamil was measured in rats using PET, and Logan analysis technique was used to calculate the DV. Logan analysis measures the radioactivity of the analyte drug in the region of interest, and the DV is calculated as the slope of the Logan plot. MRI imaging has been used to determine the efflux kinetics after focused ultrasound induced BBB opening. The efflux was found to drop in the FUS-exposed regions, and slowly recovered in a time dependent manner [19].

\subsection{Flow- vs. Perfusion-Limited Blood-Brain Barrier Transport}

Simple diffusion of compounds across the BBB occurs either paracellularly (between the cells), or transcellularly (through the endothelial cells) [2, 21]. Hydrophilic compounds frequently rely on paracellular diffusion due to their poor ability to penetrate the lipid bilayer of the endothelial cell membrane. However, the presence of tight junctions between ECs greatly limits this process. For a compound to cross into the brain transcellularly, it requires an optimal balance between lipophilicity and hydrophilicity to cross the lipid bilayers of the cells as well as the aqueous cytosol. The Lipinski's 'Rule of 5' assists in the prediction of a compound's BBB permeability. According to this general rule, compounds with fewer than $5 \mathrm{H}$-bond donors, fewer than $10 \mathrm{H}$-bond acceptors, a molecular weight less than 500 daltons, and a calculated partition coefficient $(\log P)$ value less than 5 are good candidates for BBB permeability [22-24]. The logP, determined as the octanol-water partition coefficient of a molecule, denotes its 
lipophilicity. Generally, a direct relation exists between the passive permeability of a compound across the BBB, and its logP value [25]. This proportionality may not hold true in the case of hydrophilic compounds that undergo transport through specific channels, or lipophilic compounds that are subject to active efflux. Compounds with high lipid solubility can traverse the BBB via simple diffusion process; their entry into the brain is less limited by their physicochemical properties, or carrier-based transport. As a result, the limiting step for the entry of these molecules into the brain is the velocity at which they are supplied to the BBB interface by the blood. Such compounds are said to have a flow-limited BBB permeability. Examples of these compounds include ethanol and diazepam. Of note, these flow-limited compounds are typically used as a measure of cerebral blood flow. Conversely, as a compound's logP decreases or becomes more negative, its lipid partitioning decreases and, therefore, exhibits a reduction in passive BBB permeability. Their entry into the brain tissue is not dependent on blood flow, and instead depends on their permeability across the BBB, which is indirectly dictated by their physicochemical properties. Such compounds are said to have a permeability limited BBB transport. The transport of solutes occurs over the entire area of the capillary network, and thus to take the surface area into account, the product of permeability and surface area is often used to describe the measure of solute exchange across the BBB, instead of the permeability coefficient alone [25]. Assuming a unidirectional diffusion of solute, the concentration of solute extracted from blood flowing through brain capillaries 
correlates with the permeability surface area product using the Renkin-Crone equation as follows (equation 5):

$$
E=1-e^{-P A / F}
$$

where $E$ is the total solute extraction from blood, $P$ is the solute permeability $(\mathrm{cm} / \mathrm{s}), A$ is the total capillary surface area ( $\mathrm{cm} 2 / \mathrm{g}$ of brain), and $\mathrm{F}$ is the total blood flow (cm3/s/g of brain). The above equation can be rearranged solving for the permeability surface area product, PA (equation 6):

$$
P A=-F \ln (1-E)
$$

The unidirectional transfer coefficient, Kin $(\mathrm{cm} / \mathrm{s} / \mathrm{g})$ can be represented as the product of solute extraction and blood flow (equation 7).

$$
K_{\text {in }}=F \times E
$$

when the PA values are high (PA/F >> 1), and Kin approaches F, solute entry is blood flow limited. When the PA values are low (PA/F $<<1)$, and Kin approaches the permeability surface area product, solute extraction from blood is independent of blood flow and is considered diffusion limited, as depicted in Figure 3 [26-29]. The values of PA can range between $10-4$ and $10-8 \mathrm{~cm} / \mathrm{s}$. Higher PA values of $10-5$ to $10-4 \mathrm{~cm} / \mathrm{s}$ are observed for solutes with a flow- 
limited transport such as ethanol, caffeine, antipsychotic drugs, and many CNS depressants. Diffusion limited hydrophilic solutes, such as sucrose and mannitol, exhibit PA values several orders of magnitude less, frequently in the ranges of $10-7$ and $10-8 \mathrm{~cm} / \mathrm{s}[30]$.

\subsection{Preclinical Measurements of Blood-Brain Barrier Permeability in Pathological Conditions}

Historically, measurements of BBB permeability have been achieved through multiple methodologies. These approaches include the indicator-diffusion, the brain uptake index, the concentration profile analysis, the isolated perfused brain, the intravenous injection, the in situ brain perfusion, and the multiple-time uptake techniques $[13,14,29,31-36]$. Each of these methodologies presents its own limitations ranging from inappropriate assumptions regarding tracer and blood mixing, to inaccurate estimations of poorly or rapidly penetrating solutes, and extensive animal surgery [14]. The in situ brain perfusion is capable of estimating transfer coefficients and evaluating barrier integrity with high fidelity $[14,26,37-$ 41]. However, this technique presents limitations regarding its ability to yield reproducible results in disease states with a heterogeneous disruption of the BBB (i.e., brain tumors, stroke, Alzheimer's disease, etc.). To ascertain these subtle, variable changes in BBB integrity, the single-uptake approach is widely recognized as the preferred methodology [36, 42]. 
The unidirectional transfer constant, Kin, in single-uptake experiments following an intravenous injection of the solute of interest is defined by the relationship in equation $8[9,43-45]$ :

$$
K_{i n}=\frac{C_{b r}(\tau)}{\int_{0}^{t} C_{b l}(\tau) d t}
$$

where $\mathrm{Cbr}$ is the concentration of tracer contained in the brain compartment of interest at time $\mathrm{T}$, and $\mathrm{Cbl}$ is the concentration of solute in blood. The denominator of this expression solves for the area under the curve of the change in plasma concentration from time 0 to time $\mathrm{T}$ and indicates total exposure to the solute through the duration of the experiment. The integral of the plasma concentration versus time curve is necessary because the concentration of the test solute in blood changes over time as a result of metabolism and clearance of the tracer. $\mathrm{Cbr}$ is the total concentration of measurable solute that has left the vascular compartment and distributes to the brain compartment, which is also expressed as the total quantity in brain as follows (equation 9):

$$
C_{t o t}=C_{b r}+C_{v a s}
$$

where Ctot is the total concentration of solute in the brain vascular compartments, and Cvas is the concentration of solute in the vascular space within the brain. Subtraction of the measured Cvas from Ctot provides a reliable 
estimate of $\mathrm{Cbr}$, or the quantity of tracer distribution into brain for a given period of circulation time and unit of tissue mass.

While the pharmacokinetic evaluations in this review provide an estimate of the unidirectional transfer constant for a solute, it has limited insight into the amount of unbound drug in both the blood and brain at a specific time. When considering pharmacokinetics of a solute's transport from blood to brain it is important to understand that only unbound solute can permeate across the BBB, and the unbound concentration of solute is what drives pharmacodynamic activities [46]. To determine this, an equilibrium micro-dialysis method is used, where a semipermeable probe is inserted into a specific brain region and perfusate is flowed through an interior probe and allowed to passively diffuse across the outer semipermeable membrane. The dialysate is then measured by collection from the outlet tube $[47,48]$. Briefly the equilibrium constant $\mathrm{K}$, p,uu (unbound partition coefficient) is determined as follows in equation 10:

$$
K_{p, u u}=\frac{A U C_{u, \text { brain } I S F}}{A U C_{u, p l a s m a}}
$$

where $A \cup C u$,brain and $A \cup C u$,plasma represents the total exposure of unbound drug in brain and plasma, respectively [48, 49]. Determining Kp,uu provides information on the concentration of drug freely able to act within the brain parenchyma. This measure accounts for tissue binding affinity and the properties of active and passive transport across the BBB [50], though it does not directly measure BBB transport constants. Values of $\mathrm{Kp}$, uu are reported to range from as 
low as 0.02 and 3. Contextually, solutes with high BBB permeability/equilibrium such as diazepam and oxycodone have a $\mathrm{Kp}$, uu value of 1 and 3 , respectively [51, 52]. Conversely, baclofen and morphine, solutes with poor BBB penetration and equilibrium, have a reported $\mathrm{Kp}$,uu values of 0.02 and 0.29 respetively [53, 54].

\subsection{Clinical BBB PK in Disease States and Preclinical Model Translatability} Measurement of BBB permeability and disruption in humans is not as direct as preclinical models but is readily achieved with advanced imaging techniques such as dynamic contrast-enhanced MRI (DCE-MRI), often employed in oncology and stroke imaging studies [55-58]. This type of imaging provides researchers and clinicians with estimates of Ktrans to quantify BBB permeability. As defined by Tofts, Ktrans, with units of min-1 (or time-1), is a volume transfer constant between blood plasma and extravascular, extracellular space, predominantly intended for use with tracers that do not readily enter intracellular compartments (i.e., non-lipophilic tracers) [59]. Measures of Ktrans in DCE-MRI studies are often calculated utilizing the extended Tofts-Kety (ETK) model, but can also be estimated from linearized Patlak plots of concentration versus time data; however, this method assumes negligible backflow of contrast agent from extravascular spaces into blood vessels during the scanning period [59-62]. Unlike Kin values, Ktrans is expressed in units of time-1 because each concentration term is based solely on volumetric signal and cannot be normalized to brain tissue mass, as is the case for preclinical determinations of 
Kin. The ETK and linearized Patlak model equations frequently used in this setting are displayed below (equations 11 and 12) [59, 60, 63]:

$$
\begin{aligned}
& C_{b r}(t)=K_{\text {trans }} \int_{0}^{t} C_{b r}(\tau) e^{-k_{e l}(t-\tau)} d \tau+f_{v a s c} C_{b l}(t) \text { (ETK Model) } \\
& C_{b r}(t)=K_{\text {trans }} \int_{0}^{t} C_{b r}(\tau) d \tau+f_{v a s c} C_{b l}(t) \text { (Linearized Patlak Model) }
\end{aligned}
$$

where $\mathrm{Cbr}$ is the concentration of contrast agent in the brain compartment, $\mathrm{Cbl}$ is concentration in the blood, fvasc is the volume fraction of vasculature in the tissue, and kel is the elimination rate constant from brain to blood compartments. In the linearized Patlak model, the elimination rate constant is ignored as discussed previously. After pre- and post-contrast infusion scans are obtained, non-linear (ETK) and linear (Patlak) least squares regression of these parametric equations are used to estimate Ktrans. The estimates of permeability changes or barrier disruption provide important clinical implications regarding many disease states, including cancer and stroke.

Brain tumors, whether primary or metastatic, heterogeneously disrupt local brain microvascular architecture and function which results in variable increases to passive permeability of the blood-tumor barrier (BTB) [64, 65]. Notably, measures of Ktrans in human gliomas are often elevated by orders of magnitude compared to healthy contralateral brain tissue and has been shown to correlate with glioma grade $[60,66]$. Trends in permeability increases, indicated by foldchanges in Kin values for lesioned versus normal brain are also observed in 
mouse models of glioma and breast cancer brain metastasis [36, 67, 68].

Agreement in trends between the estimates of fold-enhancement indicates that these mouse models faithfully capture the important underlying factors that dictate BBB permeability changes observed in humans.

In the case of acute ischemic stroke, clinical studies employing DCE-MRI have found significant increases in the value of Ktrans in affected regions compared to normal contralateral areas of brain parenchyma [69]. Elevated fold changes in Ktrans between affected and unaffected regions (in one study, approximately 3.5 for early post-stroke and approximately 23 for $5-7$ day follow-up) [69] are similar in magnitude to the changes in relative permeability of the BBB to dye observed in a rat model of ischemic stroke (approximately 15 fold change) [70]. This indicates that BBB permeability measurements in rodent stroke models effectively mimic the types of changes in permeability between stroke-affected and unaffected brain regions in human.

Previously discussed disease states provide validation and justification for the continued use of mouse and rat models due to their observed pathophysiological mimicry to clinically observed BBB function in these disease settings. While absolute values observed in these preclinical models do not scale directly to clinical values, the observed fold-changes in BBB permeability appear to translate consistently. Preclinical experiments studying BBB permeability across various diseases should be designed in light of the importance of appropriate normal parenchymal controls, as such measures set the baseline for 
determination of clinically translatable and meaningful fold-change measurements.

Interestingly, the magnitude of fold-change is notably different among preclinical and clinical determinations of passive permeability. One proposed source of variance that may be distinct other than the mathematics applied to each model is the tracer used in each study. Tracer or particle charge, size, polar surface area, among other properties, all variably affect BBB transport. Keeping in mind these parameters, a difference in fold-change of 9.2 for TxRed and 47.7 for GdDPTA both in a glioma model may not be all that different given the difference in physiology of mice and humans, as well as the immune system status in various animal models. Both provide measures of barrier damage, but also are indicative of the size, charge, and other chemical properties of that molecule. Figure 4 shows the difference in uptake of three distinct solutes detected through three separate imaging modalities. The BBB is consistent from species to species, at least in the case of humans and small rodents regarding cellular makeup and the rate of uptake of solutes at the BBB. However, what does change among specifies is the specific transporter composition (i.e., BCRP, P-gp, MRP1, etc.) at the BBB. While these data may indicate differences among methods, other correlates of animal and human data can be provided regarding therapeutic efficacy and brain tumors. 


\subsection{Conclusions}

The BBB dictates the kinetics of solute transfer into and out of the brain, having im-plications over the extent of drug distribution and treatment efficacy. This review outlines the techniques and the mathematical models commonly used to determine solute influx and efflux across the BBB. These techniques, such as in situ perfusion and Patlak modeling have found application in preclinical as well as clinical research. Determination of the rate of drug transfer across the BBB bears great significance during the preclinical and early stage CNS drug development process. Application of such methods could help predict drug disposition, allowing for optimal treatment of CNS pathologies. Further-more, this review is limited in its capacity, largely describing the unidirectional transfer rate at which a particular solute crosses the BBB. Not described herein are other sophis-ticated methods that also aim to determine BBB transport such as the use of microdialysis and serial CSF sampling. A complex multimodal approach using a variety of uptake methodology would be suitable for a more complete understanding of BBB transport for any given solute. 


\subsection{References}

1. Daneman, R. and A. Prat, The blood-brain barrier. Cold Spring Harb Perspect Biol, 2015. 7(1): p. a020412.

2. Abbott, N.J., et al., Structure and function of the blood-brain barrier. Neurobiol Dis, 2010. 37(1): p. 13-25.

3. Xu, L., A. Nirwane, and Y. Yao, Basement membrane and blood-brain barrier. Stroke Vasc Neurol, 2019. 4(2): p. 78-82.

4. Kalliokoski, A. and M. Niemi, Impact of OATP transporters on pharmacokinetics. Br J Pharmacol, 2009. 158(3): p. 693-705.

5. Nakanishi, T. and I. Tamai, Solute carrier transporters as targets for drug delivery and pharmacological intervention for chemotherapy. J Pharm Sci, 2011. 100(9): p. $3731-50$.

6. Mergenthaler, P., et al., Sugar for the brain: the role of glucose in physiological and pathological brain function. Trends Neurosci, 2013. 36(10): p. 587-97.

7. Zhao, Z., et al., Establishment and Dysfunction of the Blood-Brain Barrier. Cell, 2015. 163(5): p. 1064-1078.

8. Sprowls, S.A., et al., Improving CNS Delivery to Brain Metastases by BloodTumor Barrier Disruption. Trends Cancer, 2019. 5(8): p. 495-505.

9. Patlak, C.S., R.G. Blasberg, and J.D. Fenstermacher, Graphical evaluation of blood-to-brain transfer constants from multiple-time uptake data. J Cereb Blood Flow Metab, 1983. 3(1): p. 1-7.

10. Lockman, P.R., R.J. Mumper, and D.D. Allen, Evaluation of blood-brain barrier thiamine efflux using the in situ rat brain perfusion method. J Neurochem, 2003. 86(3): p. 627-34. 
11. Manda, V.K., et al., Nicotine and cotinine increases the brain penetration of saquinavir in rat. J Neurochem, 2010. 115(6): p. 1495-507.

12. Thomas, F.C., et al., Uptake of ANG1005, a novel paclitaxel derivative, through the blood-brain barrier into brain and experimental brain metastases of breast cancer. Pharm Res, 2009. 26(11): p. 2486-94.

13. Adkins, C.E., et al., P-glycoprotein mediated efflux limits substrate and drug uptake in a preclinical brain metastases of breast cancer model. Front Pharmacol, 2013. 4: p. 136.

14. Takasato, Y., S.I. Rapoport, and Q.R. Smith, An in situ brain perfusion technique to study cerebrovascular transport in the rat. Am J Physiol, 1984. 247(3 Pt 2): p. H484-93.

15. Loscher, W. and H. Potschka, Blood-brain barrier active efflux transporters: ATPbinding cassette gene family. NeuroRx, 2005. 2(1): p. 86-98.

16. de Lange, E.C., Potential role of $A B C$ transporters as a detoxification system at the blood-CSF barrier. Adv Drug Deliv Rev, 2004. 56(12): p. 1793-809.

17. Ohtsuki, S., et al., Quantitative targeted absolute proteomics-based ADME research as a new path to drug discovery and development: methodology, advantages, strategy, and prospects. J Pharm Sci, 2011. 100(9): p. 3547-59.

18. Agarwal, S., et al., Breast cancer resistance protein and P-glycoprotein in brain cancer: two gatekeepers team up. Curr Pharm Des, 2011. 17(26): p. 2793-802.

19. Chai, W.Y., et al., Magnetic-resonance imaging for kinetic analysis of permeability changes during focused ultrasound-induced blood-brain barrier opening and brain drug delivery. J Control Release, 2014. 192: p. 1-9.

20. Bart, J., et al., Quantitative assessment of P-glycoprotein function in the rat blood-brain barrier by distribution volume of [11C]verapamil measured with PET. Neuroimage, 2003. 20(3): p. 1775-82. 
21. Preston, J.E., N. Joan Abbott, and D.J. Begley, Transcytosis of macromolecules at the blood-brain barrier. Adv Pharmacol, 2014. 71: p. 147-63.

22. Lipinski, C.A., et al., Experimental and computational approaches to estimate solubility and permeability in drug discovery and development settings. Adv Drug Deliv Rev, 2001. 46(1-3): p. 3-26.

23. Vendel, E., V. Rottschafer, and E.C.M. de Lange, The need for mathematical modelling of spatial drug distribution within the brain. Fluids Barriers CNS, 2019. 16(1): p. 12.

24. Geldenhuys, W.J., et al., Molecular determinants of blood-brain barrier permeation. Ther Deliv, 2015. 6(8): p. 961-71.

25. Bradbury, M.W., The blood-brain barrier. Transport across the cerebral endothelium. Circ Res, 1985. 57(2): p. 213-22.

26. Smith, Q.R. and Y. Takasato, Kinetics of amino acid transport at the blood-brain barrier studied using an in situ brain perfusion technique. Ann N Y Acad Sci, 1986. 481: p. 186-201.

27. Robinson, P.J., Measurement of blood-brain barrier permeability. Clin Exp Pharmacol Physiol, 1990. 17(12): p. 829-40.

28. Pardridge, W.M., CSF, blood-brain barrier, and brain drug delivery. Expert Opin Drug Deliv, 2016. 13(7): p. 963-75.

29. Jain, R., et al., Quantitative estimation of permeability surface-area product in astroglial brain tumors using perfusion CT and correlation with histopathologic grade. AJNR Am J Neuroradiol, 2008. 29(4): p. 694-700.

30. Wong, A.D., et al., The blood-brain barrier: an engineering perspective. Front Neuroeng, 2013. 6: p. 7.

31. Oldendorf, W.H., Brain uptake of radiolabeled amino acids, amines, and hexoses after arterial injection. Am J Physiol, 1971. 221(6): p. 1629-39. 
32. Patlak, C.S. and J.D. Fenstermacher, Measurements of dog blood-brain transfer constants by ventriculocisternal perfusion. Am J Physiol, 1975. 229(4): p. 877-84.

33. Raichle, M.E., et al., Blood-brain barrier permeability of 11C-labeled alcohols and 150-labeled water. Am J Physiol, 1976. 230(2): p. 543-52.

34. Crone, C., The Permeability of Capillaries in Various Organs as Determined by Use of the 'Indicator Diffusion' Method. Acta Physiol Scand, 1963. 58: p. 292305.

35. Ohno, K., K.D. Pettigrew, and S.I. Rapoport, Lower limits of cerebrovascular permeability to nonelectrolytes in the conscious rat. Am J Physiol, 1978. 235(3): p. $\mathrm{H} 299-307$.

36. Mittapalli, R.K., et al., Quantitative fluorescence microscopy provides high resolution imaging of passive diffusion and P-gp mediated efflux at the in vivo blood-brain barrier. J Neurosci Methods, 2013. 219(1): p. 188-95.

37. Allen, D.D. and Q.R. Smith, Characterization of the blood-brain barrier choline transporter using the in situ rat brain perfusion technique. J Neurochem, 2001. 76(4): p. 1032-41.

38. Smith, Q.R. and D.D. Allen, In situ brain perfusion technique. Methods Mol Med, 2003. 89: p. 209-18.

39. Smith, Q.R., et al., Regional cerebrovascular transport of leucine as measured by the in situ brain perfusion technique. J Cereb Blood Flow Metab, 1985. 5(2): p. 300-11.

40. Lockman, P.R., et al., Brain uptake kinetics of nicotine and cotinine after chronic nicotine exposure. J Pharmacol Exp Ther, 2005. 314(2): p. 636-42.

41. Lockman, P.R., et al., Carrier-mediated transport of the quaternary ammonium neuronal nicotinic receptor antagonist n,n'-dodecylbispicolinium dibromide at the blood-brain barrier. J Pharmacol Exp Ther, 2008. 324(1): p. 244-50. 
42. Asotra, K., N. Ningaraj, and K.L. Black, Measurement of blood-brain and bloodtumor barrier permeabilities with [14C]-labeled tracers. Methods Mol Med, 2003. 89: p. 177-90.

43. Nakagawa, H., et al., Dexamethasone effects on [125I]albumin distribution in experimental RG-2 gliomas and adjacent brain. J Cereb Blood Flow Metab, 1987. 7(6): p. 687-701.

44. Blasberg, R.G., J.D. Fenstermacher, and C.S. Patlak, Transport of alphaaminoisobutyric acid across brain capillary and cellular membranes. J Cereb Blood Flow Metab, 1983. 3(1): p. 8-32.

45. Blasberg, R.G., et al., Local blood flow in Walker 256 metastatic brain tumors. J Neurooncol, 1984. 2(3): p. 195-204.

46. Loryan, I., M. Hammarlund-Udenaes, and S. Syvanen, Brain Distribution of Drugs: Pharmacokinetic Considerations. Handb Exp Pharmacol, 2020.

47. Hammarlund-Udenaes, M., Microdialysis as an Important Technique in Systems Pharmacology-a Historical and Methodological Review. AAPS J, 2017. 19(5): p. 1294-1303.

48. Gupta, A., et al., Brain distribution of cetirizine enantiomers: comparison of three different tissue-to-plasma partition coefficients: $K(p), K(p, u)$, and $K(p, u u)$. Drug Metab Dispos, 2006. 34(2): p. 318-23.

49. Hu, Y., et al., In Vivo Quantitative Understanding of PEGylated Liposome's Influence on Brain Delivery of Diphenhydramine. Mol Pharm, 2018. 15(12): p. 5493-5500.

50. Hammarlund-Udenaes, M., et al., On the rate and extent of drug delivery to the brain. Pharm Res, 2008. 25(8): p. 1737-50.

51. Bostrom, E., U.S. Simonsson, and M. Hammarlund-Udenaes, In vivo blood-brain barrier transport of oxycodone in the rat: indications for active influx and 
implications for pharmacokinetics/pharmacodynamics. Drug Metab Dispos, 2006. 34(9): p. 1624-31.

52. Dubey, R.K., et al., Plasma binding and transport of diazepam across the bloodbrain barrier. No evidence for in vivo enhanced dissociation. J Clin Invest, 1989. 84(4): p. 1155-9.

53. Deguchi, Y., et al., Study on brain interstitial fluid distribution and blood-brain barrier transport of baclofen in rats by microdialysis. Pharm Res, 1995. 12(12): $p$. $1838-44$.

54. Tunblad, K., E.N. Jonsson, and M. Hammarlund-Udenaes, Morphine blood-brain barrier transport is influenced by probenecid co-administration. Pharm Res, 2003. 20(4): p. 618-23.

55. Yankeelov, T.E. and J.C. Gore, Dynamic Contrast Enhanced Magnetic Resonance Imaging in Oncology: Theory, Data Acquisition, Analysis, and Examples. Curr Med Imaging Rev, 2009. 3(2): p. 91-107.

56. Mross, K., et al., DCE-MRI assessment of the effect of vandetanib on tumor vasculature in patients with advanced colorectal cancer and liver metastases: a randomized phase I study. J Angiogenes Res, 2009. 1: p. 5.

57. Leigh, R., et al., Pretreatment blood-brain barrier damage and post-treatment intracranial hemorrhage in patients receiving intravenous tissue-type plasminogen activator. Stroke, 2014. 45(7): p. 2030-5.

58. Gordon, Y., et al., Dynamic contrast-enhanced magnetic resonance imaging: fundamentals and application to the evaluation of the peripheral perfusion. Cardiovasc Diagn Ther, 2014. 4(2): p. 147-64.

59. Tofts, P.S., et al., Estimating kinetic parameters from dynamic contrast-enhanced $T(1)$-weighted MRI of a diffusable tracer: standardized quantities and symbols. J Magn Reson Imaging, 1999. 10(3): p. 223-32. 
60. Bergamino, M., et al., Measurement of blood-brain barrier permeability with $\mathrm{t} 1$ weighted dynamic contrast-enhanced MRI in brain tumors: a comparative study with two different algorithms. ISRN Neurosci, 2013. 2013: p. 905279.

61. Tofts, P.S., Modeling tracer kinetics in dynamic Gd-DTPA MR imaging. J Magn Reson Imaging, 1997. 7(1): p. 91-101.

62. Zhang, N., et al., Correlation of volume transfer coefficient Ktrans with histopathologic grades of gliomas. J Magn Reson Imaging, 2012. 36(2): p. 35563.

63. Law, M., et al., Comparison of cerebral blood volume and vascular permeability from dynamic susceptibility contrast-enhanced perfusion MR imaging with glioma grade. AJNR Am J Neuroradiol, 2004. 25(5): p. 746-55.

64. Gerstner, E.R. and R.L. Fine, Increased permeability of the blood-brain barrier to chemotherapy in metastatic brain tumors: establishing a treatment paradigm. J Clin Oncol, 2007. 25(16): p. 2306-12.

65. de Vries, N.A., et al., Blood-brain barrier and chemotherapeutic treatment of brain tumors. Expert Rev Neurother, 2006. 6(8): p. 1199-209.

66. Zhang, J., et al., Increasing of Blood-Brain Tumor Barrier Permeability through Transcellular and Paracellular Pathways by Microbubble-Enhanced Diagnostic Ultrasound in a C6 Glioma Model. Front Neurosci, 2017. 11: p. 86.

67. Lockman, P.R., et al., Heterogeneous blood-tumor barrier permeability determines drug efficacy in experimental brain metastases of breast cancer. Clin Cancer Res, 2010. 16(23): p. 5664-78.

68. Mohammad, A.S., et al., Permeability changes and effect of chemotherapy in brain adjacent to tumor in an experimental model of metastatic brain tumor from breast cancer. BMC Cancer, 2018. 18(1): p. 1225. 
69. Villringer, K., et al., DCE-MRI blood-brain barrier assessment in acute ischemic stroke. Neurology, 2017. 88(5): p. 433-440.

70. Fernandez-Lopez, D., et al., Blood-brain barrier permeability is increased after acute adult stroke but not neonatal stroke in the rat. J Neurosci, 2012. 32(28): p. $9588-600$. 


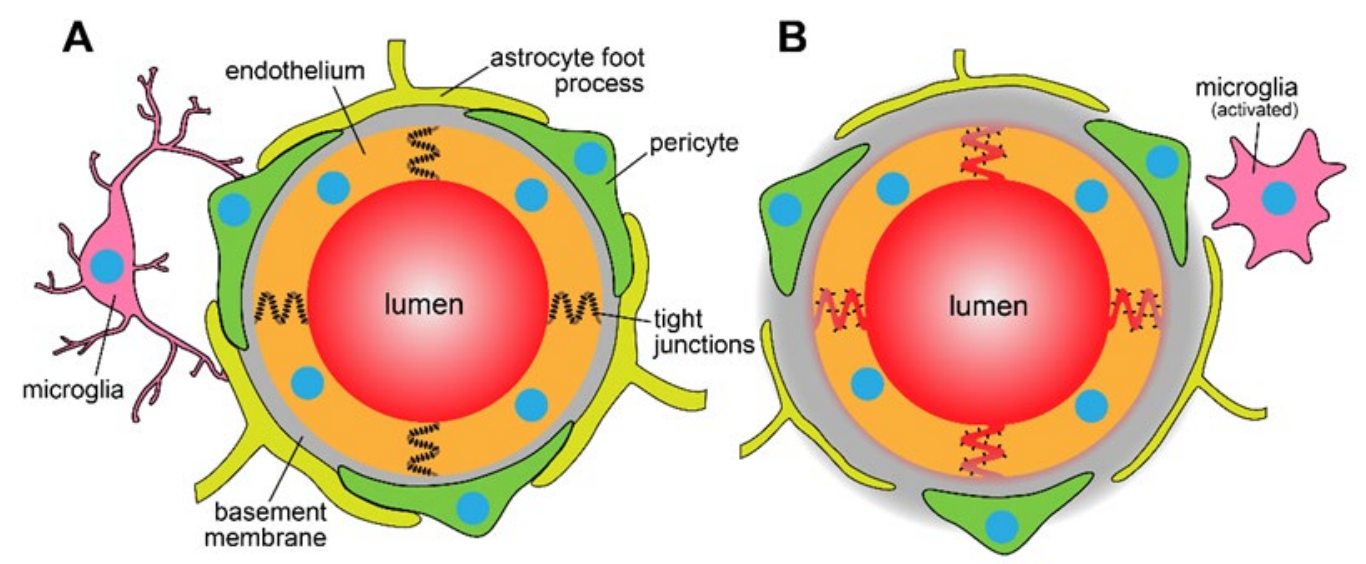

Figure 6.1. Anatomical differences between (A) blood-brain barrier vasculature and (B) disrupted vasculature. The BBB is characterized by presence of endothelial tight junctions, formed by the tight junction proteins and the adjacent pericytes, microglia and astrocytic foot processes. 


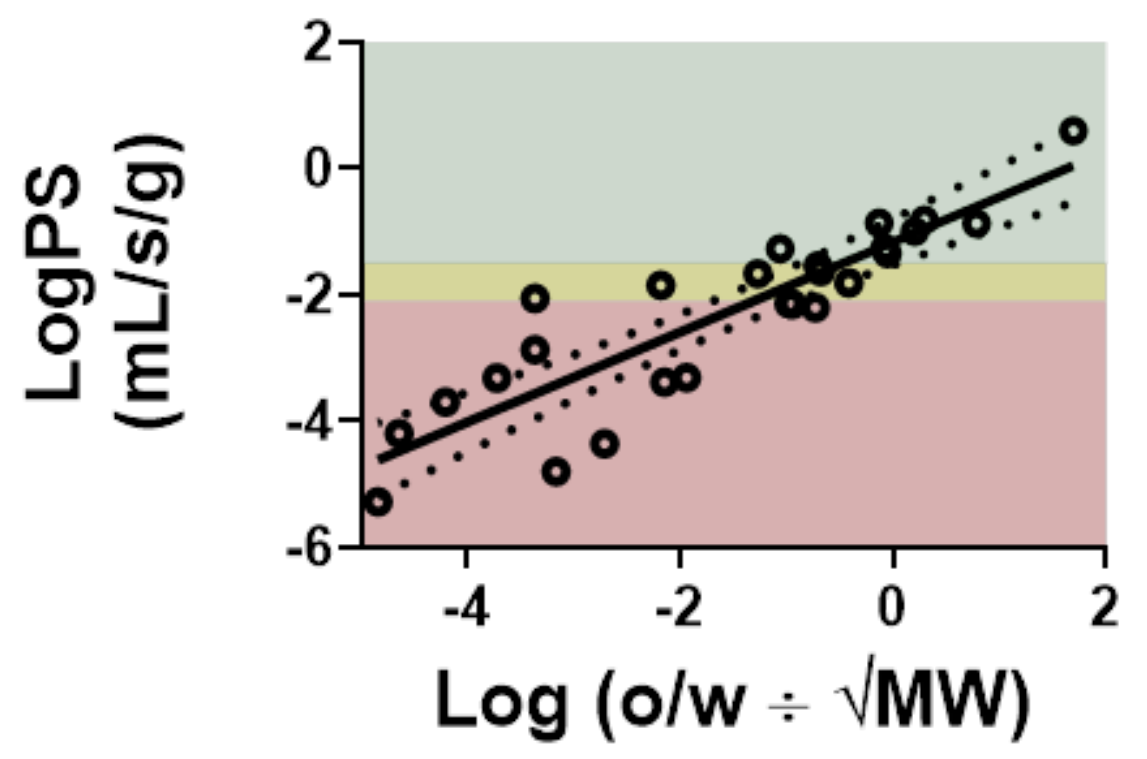

Figure 6.2. Correlation of solute BBB permeability, indicated by its permeability surface area (PS) product, with the $\log (P \div \sqrt{M W})$. Compounds with higher lipophilicity have a greater tendency to traverse the BBB. Compounds in the green-shaded area are those with values of $80 \%$ of reported cerebral blood flow or high. Compounds in the yellow shaded region indicate those with PS values between 20 and $80 \%$ of cerebral blood flow. Compounds with PS values in the red-shaded area are those with reported PS values which are less than $20 \%$ of cerebral blood flow. Values compiled from literature reported values of PS [14-17] R2 $=0.78$ 

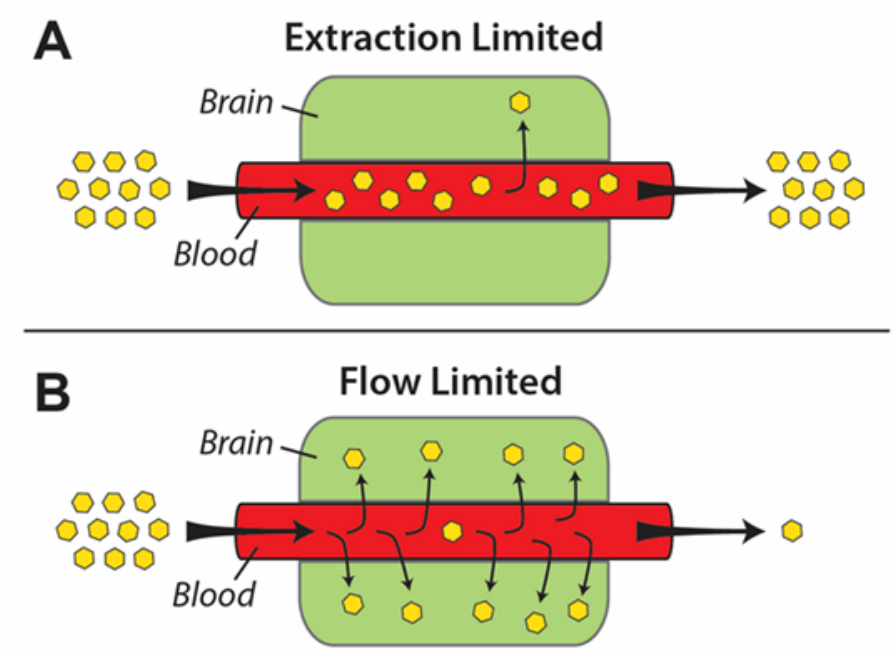

Figure 6.3. A schematic representation of (A) extraction-limited and (B) flowlimited solute transfer across the BBB. The physicochemical properties of compounds having extraction-limited permeability are not amenable to BBB transport. Conversely, the transport of highly permeable solutes across the BBB is generally quick, and only limited by how rapidly they are presented to the BBB. 

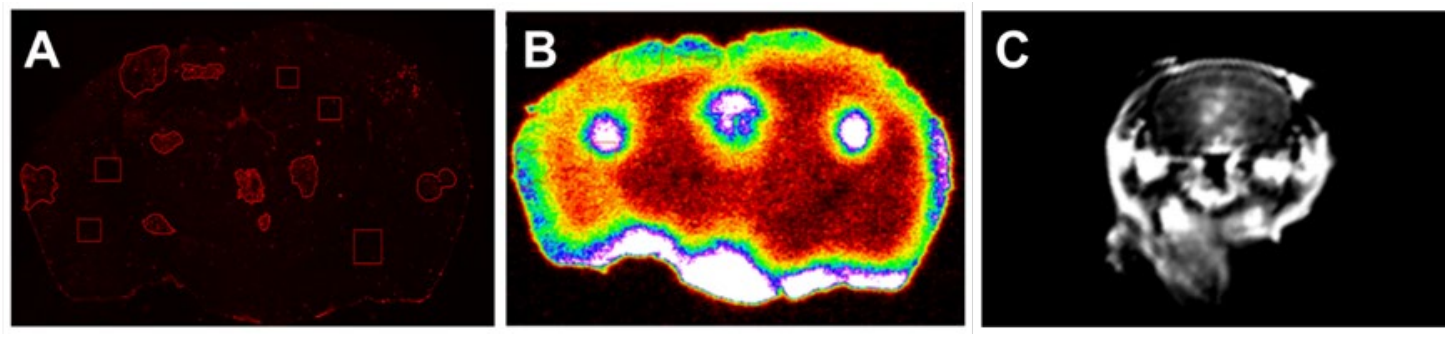

Figure 6.4. Differential tracer uptake in various imaging modalities.

Accumulation of Texas Red 3K (A) and 14C-aminoisobutyric acid (B) in brain metastases of breast cancer using fluorescent and phosphorescent quantitative imaging. (C)T1 cortical Turbo Spin Echo MRI indicating gadavist enhancement in lesions within the brain. 


\section{Chapter 7}

\section{Conclusions and Future Directions.}

\subsection{Conclusions}

In conclusion, this dissertation provided a detailed review on the blood-brain barrier, brain metastases, and treatment strategies to overcome brain barriers during the management of metastatic and primary brain tumors. Herein, we also evaluated the efficacy of the hypomethylating agent azacitidine for treatment of breast cancer brain metastases in a preclinical setting. Additionally, we observed the effects of radiation therapy on the blood-brain and blood-tumor barriers, as well as the role cannabidiol plays in active efflux inhibition and in the management of metastatic brain tumors.

Alteration in the methylation of DNA is used by cells to drive gene expression, but also by cancer cells to suppress or overexpress different proteins driving oncogenic progression. Aberrant methylation is found in several cancer types, include breast cancer. Azacitidine has shown promise in controlling hematological malignancies, of which many have specific hypermethylated genes. We found AZA to have a significantly lower half-maximal inhibitory concentration $\left(\mathrm{IC}_{50}\right)$ in $231 \mathrm{Br}$ cells compared to 231 cells, suggesting that $231 \mathrm{Br}$ cells are more sensitive to AZA due a favorable phenotypic change allowing for increased brain tropism. AZA was also found to increase proapoptotic and 
decrease antiapoptotic proteins in the $231 \mathrm{Br}$ cells. The $231 \mathrm{Br}$ cells were found to have increased hallmark metastasis markers compared to the 231 parent cell line. The $231 \mathrm{Br}$ cells were found to have methylation of the Keratin18 gene, confirmed by restriction enzyme digestion and correlated with an increased expression of DNMT3a. This methylation could be targeted by AZA in vivo, resulting in increased survival and decreased tumor burden.

The non-psychoactive, non-toxic component of Cannabis sativa, cannabidiol, has shown anecdotal promise in the management of many cancer types, as well as in the alleviation of chemotherapy induced adverse effects. We found CBD to induce cancer cell death at an $\mathrm{IC}_{50}$ of $\sim 10 \mu \mathrm{M}$ in the $231 \mathrm{Br}$ cell line in vitro. Additionally, CBD was able to significantly increase the concentration of paclitaxel, a P-gp substrate, distributing into brain. CBD was found to reduce the formation of $231 \mathrm{Br}$ brain metastases in a prevention model indicated by statistically less bioluminescent signal accumulation over time. In these, CBD was also able to help maintain the weight of animals with brain lesions compared to vehicle treatment. In a combinational approach with paclitaxel, CBD failed to improve the survival and management of brain metastases. However, results from this experiment coincide with other literature and clinical results suggesting P-gp inhibition does not correlate with increased survival. Together, these data provide evidence of CBD's potential use in prevention of brain metastases and the management of chemotherapeutic induced weight decline. Additionally, CBD has also been shown to inhibit P-gp efflux at the BBB. 
Radiation therapy is a mainstay in the treatment of primary and metastatic cancers. The impact of radiation on the integrity of the BBB has been controversial and no defining data has been provided to support either case. In our work, we developed a rational platform for the use of radiation therapy in both in vivo and in vitro preclinical models. Using a combination of ionization chamber and gafchromic dosimetry techniques alongside immunohistochemical verification, our field was show to effectively deposit radiation doses in vivo with acceptable field homogeneity. We found the normal BBB to be undisrupted in the immune deficient athymic nude mouse model at doses up to $12 \mathrm{~Gy}$ in fractionation as suggested by Texas Red accumulation. However, in the immune competent FVB mouse model we found the normal BBB to have increased permeability 24 hours following dose of $12 \mathrm{~Gy}$ in 4 fractions. We also noted increased permeability of triple negative brain metastases treated with 6 and $12 \mathrm{~Gy}$ to have increased permeability 24 hours following radiation therapy, while only the lesions treated with $6 \mathrm{~Gy}$ demonstrated increased permeability of the BTB at 8 hours following radiotherapy. These data suggest disruption of the BBB in normal brain tissue following radiation treatment is dependent on immune system status, further indicating a possible abscopal-like response. Also, permeability of brain lesions occurs lesions treated with radiation therapy occurs in a time and dose dependent manner, which may provide for the means to more appropriately coordinate treatment modalities in patients with metastatic brain 
tumors. The last findings of this work reveal the extensive heterogeneity in the approach to studying radiation therapy in animal models.

In summary, the work from this dissertation confirms the difficulty in finding a safe, efficacious treatment for brain metastasis of breast cancer. However, the hope is that these findings will help push the field forward and provide a starting point for other researchers to branch from.

\subsection{Future Directions}

Future studies that are resultant of this work will include the following:

Azacitidine has been shown to impact both survival and tumor burden in our preclinical models of breast cancer brain metastasis. It been reported that azacitidine does cross readily cross the BBB. Reduced tumor burden and increased survival are the main outcomes desired during rationale drug delivery approaches, but AZA has also been described to have unintended hematological toxicities. Future studies evaluating the dose of AZA being used should be performed. The unidirectional transfer constant $\left(K_{\text {in }}\right)$ of AZA will also be assessed using our in situ brain perfusion technique with radiolabeled AZA.

Literature and anecdotal reports of the use of CBD during cancer treatments have provided some insight into its potential as a stand-alone or combinatorial 
cancer therapeutic. The data herein indicate that CBD inhibits efflux at the BBB through in situ brain perfusions. However, in our efficacy study results in combination with the P-gp substrate paclitaxel did not indicate any modest increase in survival or decrease in tumor burden. Future studies for this line of study will include a second set of in situ brain perfusions with the addition of capillary depletion sub experiments to evaluate the true position paclitaxel. The results from this experiment will demonstrate whether accumulation of paclitaxel after P-gp inhibition is truly in the brain parenchyma or if it is sequestered in the capillaries within the brain. Another future study, will evaluate the capability of CBD to reduce the neurotoxic adverse effects due to radiation therapy such as cognitive deficits and glial scarring.

The widespread use of radiation therapy in the management of metastatic and primary brain tumors requires that we need to better understand the pathophysiological outcomes of this therapy. The BBB has been shown by some and by our work to be disrupted following treatment with ionizing radiation. The extent, magnitude, and time course of this disruption are not well defined. However, the work herein presents a platform to evaluate these lines of thought. Also, the immune system plays a clear role in the BBB's response to radiation exposure. Future studies will utilize our multimodal fluorescent and phosphorescent imaging techniques to evaluate the extent and magnitude of barrier opening at several time points post radiation therapy. Additionally, the immune cell population and cytokine pool within the brain 
at each of these time points will be evaluated by dedicated flow cytometry analysis and a MSD inflammatory marker assay. Additionally, using the applied pharmacokinetics reviewed in Chapter 6, we will quantitatively measure the unidirectional transfer constant, $\mathrm{K}_{\mathrm{in}}$, following radiation therapy, as well as the extent to which uptake of nutrients is impacted following treatment.

Novel treatment strategies have progressed the management of primary breast and other cancers dramatically in recent years. The unintended consequence of this success in the increase in prevalence of brain metastases. Novel drug formulations and disruptive methods aimed at bypassing the blood-brain and blood-tumor barriers provide an optimistic outlook for the treatment of metastatic brain lesions.

Reframing the aims of my work during my time as a post-doc, I will be focusing on primary brain tumors, specifically glioblastoma (GBM). Using the techniques and training, I have received at WVU I will be investigating the impact radiation therapy has on the blood-tumor barrier in primary GBM, stratified across biological sex. Lathia and colleagues have observed a more activated microglial phenotype and decreased survival in female mice deficient in the junctional adhesion molecule-A. The observed sexual dimorphism that exists is intrinsically located within a key cellular component of the blood-brain barrier. We will aim to identify other novel sex differences at the blood-brain and blood-tumor barriers, but also the extent to which they impact the therapeutic efficacy of radiation 
therapy, and to what extent permeability is increased following treatment with ionizing radiation. 
